SANDIA REPORT

SAND2003-3408

Unlimited Release

Printed January 2004

\title{
Continuous-Wave Radar to Detect Defects Within Heat Exchangers and Steam Generator Tubes*
}

Gary E. Rochau, Thurlow W. H. Caffey, Bahram Nassersharif, Gabe V. Garcia, and Russell P. Jedlicka

Prepared by

Sandia National Laboratories

Albuquerque, New Mexico 87185

Sandia is a multiprogram laboratory operated by Sandia Corporation, a Lockheed Martin Company, for the United States Department of Energy under contract DEAC04-94AL85000.

Approved for public release; further dissemination unlimited.

\section{Sandia National Laboratories}




\section{Issued by Sandia National Laboratories, operated for the United States Department of Energy by Sandia Corporation.}

NOTICE: This report was prepared as an account of work sponsored by an agency of the United States Government. Neither the United States Government, nor any agency thereof, nor any of their employees, nor any of their contractors, subcontractors, or their employees, make any warranty, express or implied, or assume any legal liability or responsibility for the accuracy, completeness, or usefulness of any information, apparatus, product, or process disclosed, or represent that its use would not infringe privately owned rights. Reference herein to any specific commercial product, process, or service by trade name, trademark, manufacturer, or otherwise, does not necessarily constitute or imply its endorsement, recommendation, or favoring by the United States Government, any agency thereof, or any of their contractors or subcontractors. The views and opinions expressed herein do not necessarily state or reflect those of the United States Government, any agency thereof, or any of their contractors.

Printed in the United States of America. This report has been reproduced directly from the best available copy.

Available to DOE and DOE contractors from

U.S. Department of Energy

Office of Scientific and Technical Information

P.O. Box 62

Oak Ridge, TN 37831

$\begin{array}{ll}\text { Telephone: } & (865) 576-8401 \\ \text { Facsimile: } & (865) 576-5728 \\ \text { E-Mail: } & \text { reports@adonis.osti.gov }\end{array}$

Online ordering: http://www.doe.gov/bridge

Available to the public from

U.S. Department of Commerce

National Technical Information Service

5285 Port Royal Rd

Springfield, VA 22161

Telephone: $\quad$ (800)553-6847

Facsimile: $\quad$ (703)605-6900

E-Mail: $\quad$ orders@ntis.fedworld.gov

Online order: http://www.ntis.gov/help/ordermethods.asp?loc=7-4-0\#online 
SAND2003-3408

Unlimited Release

Printed January 2004

\title{
Continuous-Wave Radar to Detect Defects Within Heat Exchangers and Steam Generator Tubes*
}

\author{
Revised September 3, 2003 \\ Gary E. Rochau and Thurlow W. H. Caffey \\ Sandia National Laboratories, \\ Albuquerque, New Mexico 87185-0740 \\ Bahram Nassersharif and Gabe V. Garcia \\ Department of Mechanical Engineering New Mexico State University, \\ Las Cruces, NM 88003-8001 \\ Russell P. Jedlicka \\ Klipsch School of Electrical and Computer Engineering New Mexico State University, \\ Las Cruces, NM 88003-8001 \\ *The work reported herein was performed at Sandia under NERI reference \# 99-0308, \\ and at New Mexico State University under NERI reference \# DE-FG03-99SF21986.
}

\begin{abstract}
A major cause of failures in heat exchangers and steam generators in nuclear power plants is degradation of the tubes within them. The tube failure is often caused by the development of cracks that begin on the outer surface of the tube and propagate both inwards and laterally. A new technique was researched for detection of defects using a continuous-wave radar method within metal tubing. The experimental program resulted in a completed product development schedule and the design of an experimental apparatus for studying handling of the probe and data acquisition. These tests were completed as far as the prototypical probe performance allowed. The prototype probe design did not have sufficient sensitivity to detect a defect signal using the defined radar technique and did not allow successful completion of all of the project milestones. The best results from the prototype probe could not detect a tube defect using the radar principle. Though a more precision probe may be possible, the cost of design and construction was beyond the scope of the project. This report describes the probe development and the status of the design at the termination of the project.
\end{abstract}




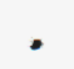

This page intentionally left blank 


\section{TABLE OF CONTENTS}

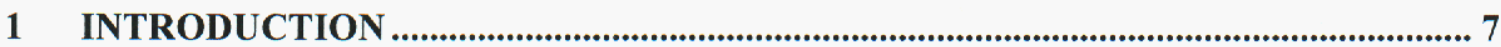

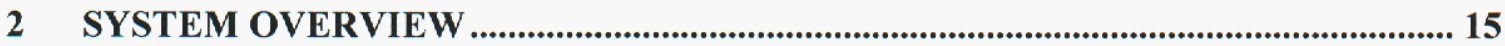

2.1 SYSTEM ARCHITECTURE AND CONCEPTUAL DESCRIPTION ….................................... 15

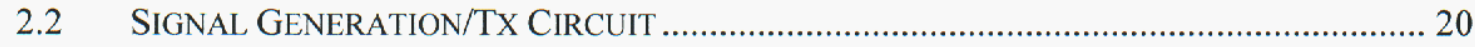

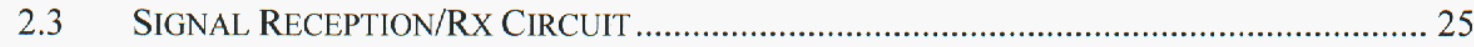

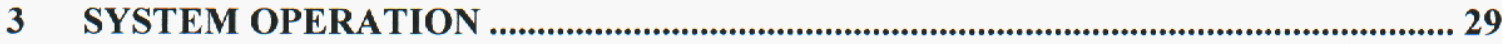

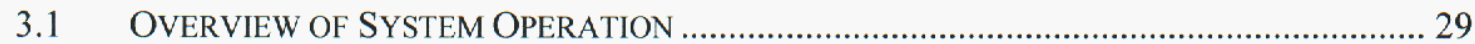

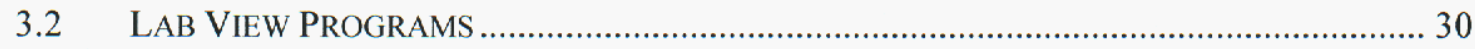

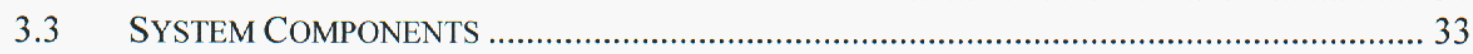

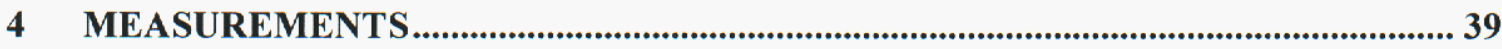

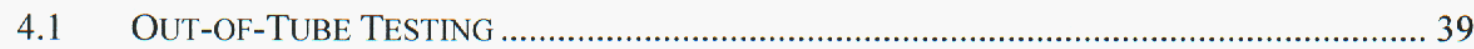

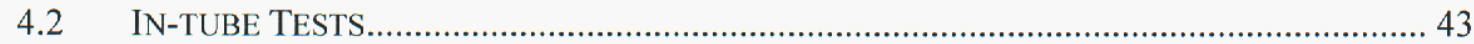

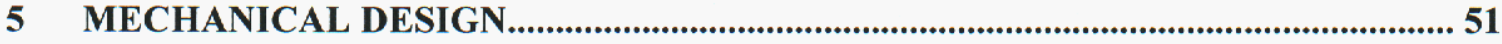

6 ELECTROMAGNETIC MODELING AND SIMULATION .......................................53

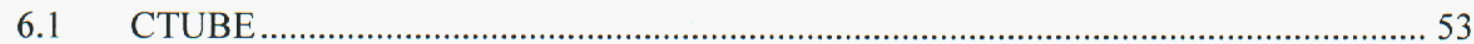

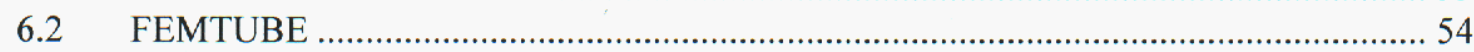

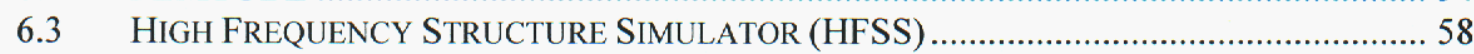

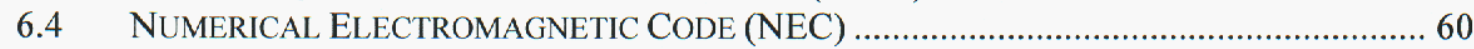

7 CONCLUSIONS AND RECOMMENDATIONS FOR FUTURE WORK ................... 65

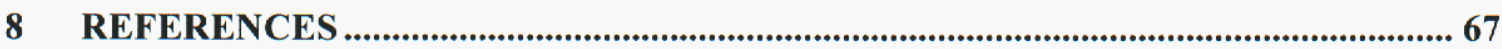

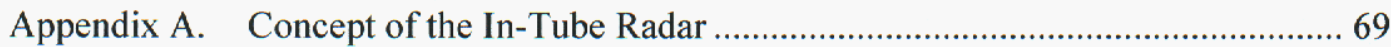

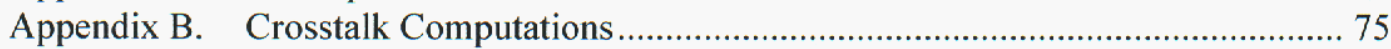


This page intentionally left blank 


\section{INTRODUCTION}

A major cause of failures in heat exchangers and steam generators in nuclear power plants is degradation of the tubes within them. The tube failure is often caused by the development of cracks that begin on the outer surface of the tube and propagate both inwards and laterally. A new technique was researched for detection of defects using a continuous-wave radar method within metal tubing. The technique is $100 \%$ volumetric, and may find smaller defects, more rapidly, and less expensively than present methods.

The project described in this report was a joint development effort between Sandia National Laboratories (SNL) and New Mexico State University (NMSU) funded by the US Department of Energy. The goal of the project was to research, design, and develop a new concept utilizing a continuous wave radar to detect defects inside metallic tubes and in particular nuclear plant steam generator tubing. The project was divided into four parallel tracks: computational modeling, experimental prototyping, thermo-mechanical design, and signal detection and analysis.

This work is sponsored by the Department of Energy's Nuclear Energy Research Initiative (Grant Number DE-FG-3- 99SF21986).

\section{Introduction to Physical Concepts}

The basic concept of the radar is to illuminate the inside of the tube with an electric field that is parallel to the tube wall but which is zero in the vicinity of the tube axis. A small portion of the field penetrates the tube wall, is back-scattered by a defect, and re-enters the tube interior [3]. The larger fraction of the field is reflected back into the interior but, by symmetry, the reflections combine to a very small value near the axis of the tube so that the signal from the defect can be detected.

The desired electric field is generated by a small loop that is centered at the origin of a Cartesian coordinate system so that the windings of the loop are in the $y$-z plane. The magnitude of the vertical electric field, or $\left|E_{z}\right|$, has the relative distribution in air as shown in Figure 1-1. 


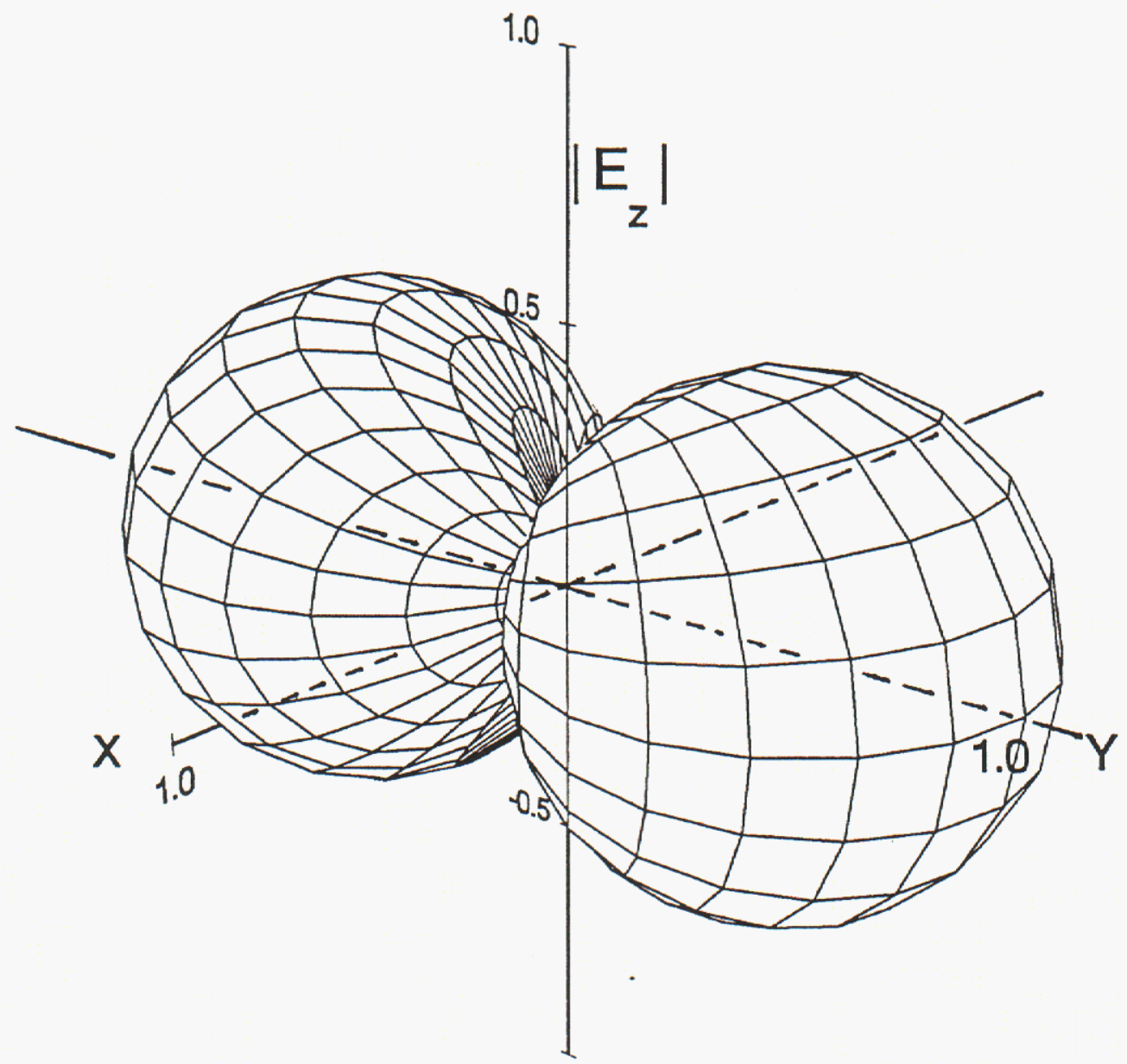

Figure 1-1. Distribution of the vertical electric field in air

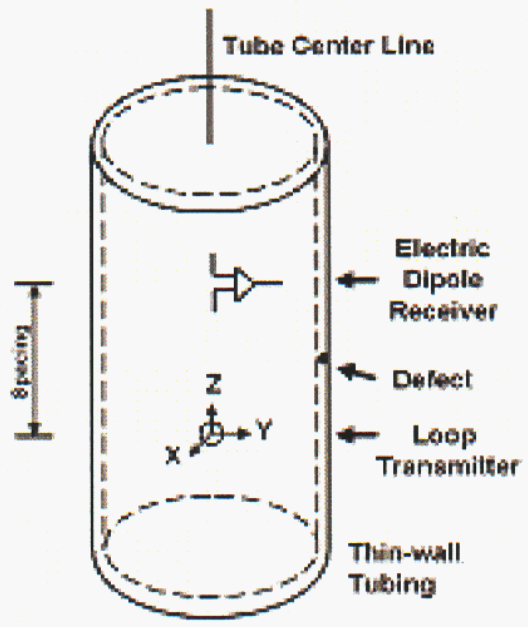

Figure 1-2.Geometry of the tube and antennas 
The $\left|E_{z}\right|$-field is zero everywhere in the xz-plane, so that an electric dipole can be placed along the z-axis without being coupled to the transmitting loop. The geometry of the loop transmitter and an electric dipole receiver within a tube is shown in Figure 1-2. The size of the defect shown in the tube wall is greatly exaggerated for illustration. The z-axis coincides with the axis of the tube, and the electric dipole is placed above the loop by a spacing which must be chosen because the distribution of $\left|E_{z}\right|$ varies with the separation distance along the z-axis.

As a specific example, consider an Inconel tube with an inside diameter of $2.2 \mathrm{~cm}$ and a wall thickness of $0.127 \mathrm{~cm}$ (0.05 inches), and examine the $\left|E_{z}\right|$-distribution throughout an xy-plane that is $2.2 \mathrm{~cm}$ above the loop source. This might be the location of the lower portion of the receiving dipole. The most favorable case occurs when the tube wall is replaced with air and this distribution is shown in Figure 1-3. The appearance is that of a folded, circular disc whose rim corresponds to the tube wall. A clean null in the xz-plane is present as before. When the metal wall is introduced, the situation changes as shown in Figure 1-4. The fold of the disc is rotated 90 degrees from the air-tube case, and is non-zero along the $y$-axis except at $y=0$. The maximum value, at the $x$-axis rim, is $26 \%$ of the maximum at the y-axis rim in Figure 1-3, and the crosscoupled field near the z-axis is very small.

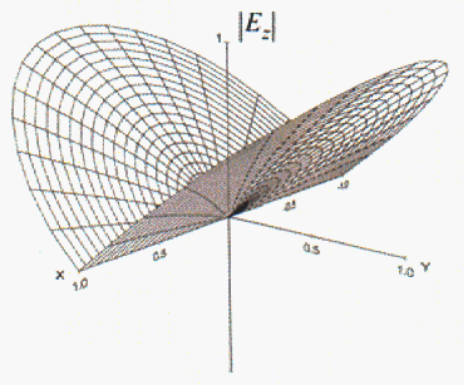

Figure 1-3. Distribution of the vertical electric field within an air tube 


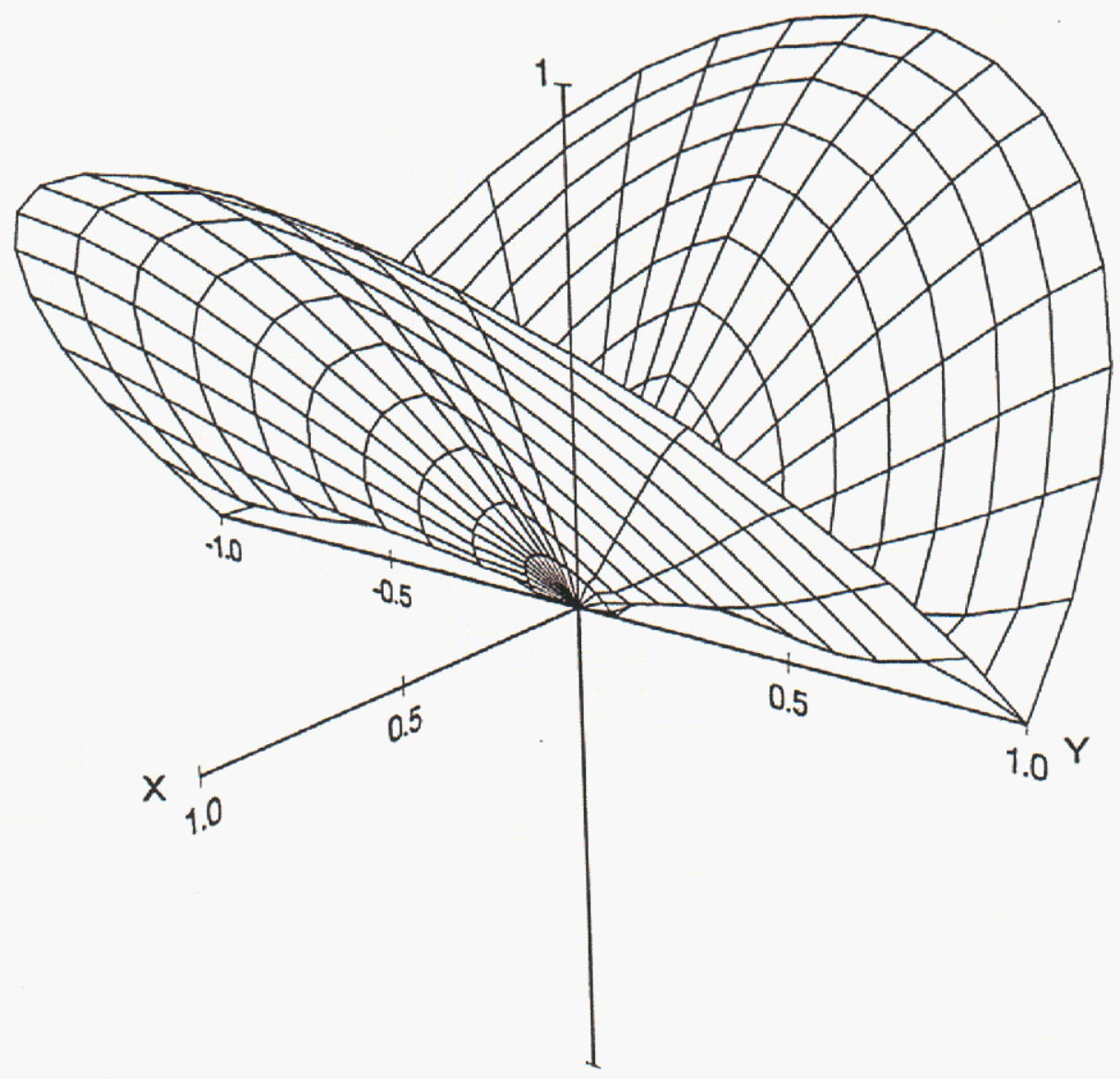

Figure 1-4. Distribution of the vertical electric field within a metal tube

\section{Numerical Modeling}

The numerical modeling of the problem was carried out through two parallel efforts; one at Sandia and the second at NMSU. Sandia had previously developed the CTUBE code[1,2] for modeling of a continuous-wave underground radar. Modifications of CTUBE to model the problem under non-symmetric conditions were implemented [5].

The numerical modeling effort at NMSU focused on further development of the NMSU finiteelement code to model cracks in heat exchanger pipes. A computer program was also developed for generation of the structured mesh to be used for the cylindrical geometry.

In addition, a commercial code, ANSOFT HFSS software, was also used to model the problem.

In all modeling effort, the electromagnetics problem proved to be too complex for all existing codes. We were not able to generate satisfactory results for the case of non-symmetric geometry.

\section{Experimental Design}

The basic operation of the In-Tube Radar (ITR) sensor is based upon transmission and reception of an electronic signal within the heat exchanger tube. This requires a transmitter to generate the 
fields and a receiver along the centerline of the tube. A reference signal is generated in the signal processing instrumentation and coupled to the sensor itself via fiber optic cable. It is amplified and alternately applied to two orthogonal loop antennas via a switching network. The transmitter produces electromagnetic fields within the tube and its wall. The frequency of operation is selected such that the two-way path through the metal wall of the heat exchanger tube is one skin depth, a total power loss of $8.8 \mathrm{~dB}$. The loop antennas are symmetrically placed within the sensor so that when either one transmits in the absence of a wall defect the electric field at the receive monopole antenna cancels producing zero volts. The presence of a crack anywhere in the wall (interior or exterior) precludes the conditions for exact cancellation of the field; an increase in voltage at the terminals of the receiver monopole indicates a defect.

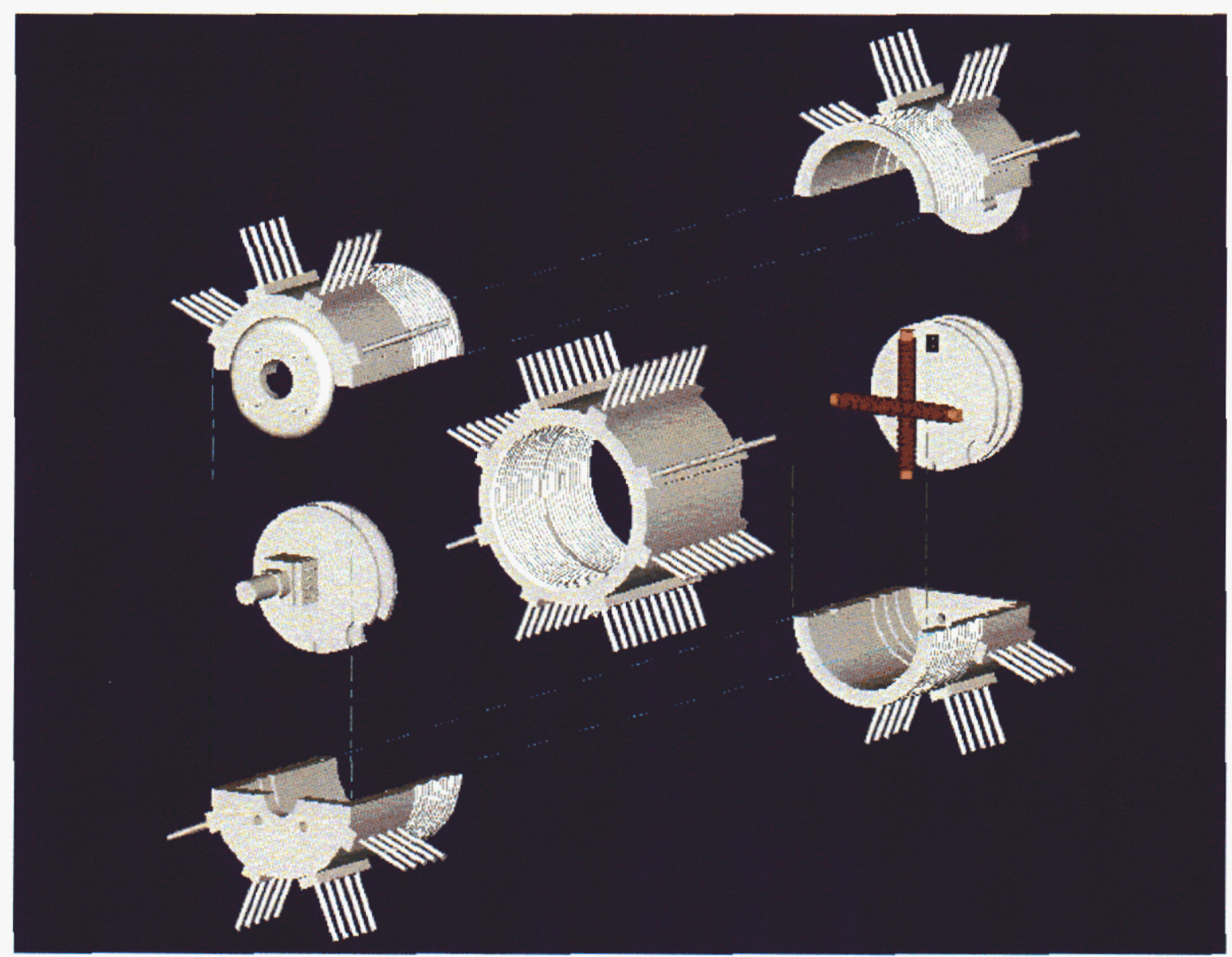

Figure 1-5. Probe assembly

Figure 1-5 shows the sensor housing for the experimental system. The associated transmitter and receiver electronics are currently under development. The testing of the experimental circuit is planned for summer 2001 .

\section{Thermo-mechanical Design}

Several concepts were considered in the thermo-mechanical design of the sensor package [6], including the ability to maintain the receiving antenna on the centerline of the tube, ability to easily adjust the separation distance between the transmitting and receiving antennas, 
electromagnetic compatibility of all materials, ease of manufacturing, and method for translating the device through a straight and curved tube. A complete solid model of the design was finished, and used as the input information for a finite element stress analysis of the device under various acceleration and loading conditions. An exploded assembly view of the mock-up probe is illustrated in Figure 1-5.

The device consists of a forward section, housing three circuit boards (data communications, transmitter amplifier, and power supply) and two wire-round ferrite rods positioned orthogonal to one another and the axis of the tube. The after section of the housing contain two boards, for receiver antenna processing and optical communications, and power supply, along with a monopole receiver antenna aligned with the tube axis. The two ends of the housing are assembled, and are threaded externally with 12-pitch threads. One end of the housing has a righthand thread, while the opposing end is threaded with a left-hand thread. This allows the outer, center section of the body to be rotated and adjust the separation distance from the transmitter to the receiver.

A series of bristles, much like hair brush bristles, was installed on the exterior surface of the device to provide a means for centering the device on-axis, while still enabling the sensor to pass through restrictions such as tube sleeves, and into the curved portion of the tube. The end cap is design to be easily removable, and the geometry can be readily adapted to mate the sensor itself with a variety of commercially available feeder mechanisms. For our initial testing, we are using an industry standard Z-TEC probe feeder.

\section{Data Acquisition System and Fiber Optic Communication}

At the start of the project, it was decided that a fiber optic system would be used for all communication between the data acquisition system and probe due to electromagnetic interference constraints. This would require three separate optical signal paths: one optical signal will be used to drive the probe antenna, the second optical signal will be used as a switch control, and the third optical signal will be the signal detected by the probe receiver antenna.

Initially, a commercial off-the-shelf voice-link fiber optic kit was evaluated. On the basis of this evaluation, a new circuit was designed, constructed, and tested. This new circuit is used to control the output signal generated by the probe antenna and for the switch. A second circuit with a highend optical transmitter and receiver was also designed. This circuit is used for the signal detected by the probe receiver antenna.

National Instruments LABVIEW software program is used for the computer/user interface and for control of the data acquisition system. Several LABVIEW programs were written to control the optical fiber system, generate the drive signal for the probe antenna, and for acquisition of the receiver antenna signal. A National Instruments $250 \mathrm{kS} / \mathrm{s}$ data acquisition board was used during this phase of the project. From these initial studies, it was determined that a $1.5 \mathrm{MS} / \mathrm{s}$ data acquisition board would be needed to acquire the receiver antenna signal. In addition to this, a $1 \mathrm{MS} / \mathrm{s}$ output board would be needed to generate the drive signal for the output antenna and to control the drive mechanism used to push and pull the probe along the tube.

\section{Signal Analysis and Processing}

The Data Acquisition System and Fiber Optic Communication team is also responsible for the interrogation of the signal detected by the probe receiver antenna. Pattern recognition will be used to detect tube damage and determine the type of damage. The idea here is to stream the receiver signal to the computer hard drive using LABVIEW and to simultaneously use a MATLAB 
software program within LABVIEW for damage detection and type. A MATLAB program was written to detect square pulses inserted in a random signal. Once actual data is available for the receiver antenna, additional MATLAB programs will be written and tested. In the meantime, we are continuing with our study of pattern recognition and how it can be used to detect and classify damage.

\section{Students Supported on This Project}

The design and engineering work in this project could not have been achieved without the dedication of many students who contributed to this project. They are listed here:

\section{Graduate Students}

Sean Brooks, completed M.S.M.E. in 2000

Edmund Rede, Completed M.S.M.E. in 2001

Sean Barley, M.S.M.E. in progress

Edward Rede, M.S.E.E. expected in 2003

\section{Undergraduate Students}

Spring 2002

David Blacklock, Industrial Engineering Gabriel Garde, Mechanical Engineering Erin Coffman, Mechanical Engineering Tomas Sanchez, Mechanical Engineering Kevin Woolfolk, Mechanical Engineering Sana Clason, Technical Communcations

\section{Fall 2002}

Marcus Bluth, Mechanical Engineering Carlos Hernandez, Industrial Engineering Rafael Padilla, Mechanical Engineering Kelli Schmitt, Mechanical Engineering Jonathan Strausbaugh, Mechanical Engineering 
This page intentionally left blank 


\section{System Overview}

The In-Tube Radar (ITR) was proposed as an alternative to the eddy current techniques presently used for heat exchanger tube inspection. In contrast to the eddy current technique, which uses two coils near the wall of the steam generator tube, the proposed ITR technique was derived from a borehole radar system developed at Sandia National Laboratories. The ITR system assumes a magnetic dipole (loop) antenna resides on the axis of the steam generator tube and generates electromagnetic fields at a frequency such that the fields penetrate the wall of the tube. Axially displaced from the loop antenna, but also on axis, is an electric antenna. In the original borehole radar the receive antenna was a dipole; to reduce size and allow shielding of the electronics it is a monopole in the ITR sensor. The borehole radar, from which the ITR concept was derived, radiated fields through the non-metallic drill casing and, in fact, operated as a radar -- operation was at a frequency such that the radiation efficiency of the transmit and receive antennas allowed a significant amount of real power to be radiated and received once scattered by objects and material discontinuities in the media surrounding the drill casing. While ITR is termed a radar, it is not one in the traditional sense; that is, it is not representative to think of it in terms of waves propagating out towards the tube walls and being reflected. Due to the frequency of operation and the fact that the metallic heat exchanger tubes are directly adjacent to both transmit and receive "antennas," the ITR system does not radiate real power. Instead, the near-field coupling between the antennas is affected in different ways by the tube wall without and with defects. Basically, it produces an induction field that, in turn, generates currents in the wall of the tube. In the absence of defects (interior or exterior), these currents are symmetric and produce a null electric field at the location of the receive dipole or monopole. The presence of a defect perturbs the symmetry with the result that the on-axis electric field will become non-zero; that is, its magnitude will increase. By observing the received signal as a function of longitudinal position within the tube (as the sensor moves) defects may be located. One of the primary differences between the ITR technique and the eddy current method is the reception of the signal. The ITR sensor uses an axial electric antenna (dipole or monopole) while the eddy current receive "antenna" is another magnetic antenna (loop). This gives the eddy current method greater coupling efficiency between the transmitter and receiver for two reasons: 1) the transmitter and receiver are in closer proximity and 2) the inductive coupling between two loops is more efficient at the required frequency of operation. However, an advantage of ITR is that it does not require comparison of the sensor output to that of one in a standard tube; it is a stand-alone device.

\subsection{System Architecture and Conceptual Description}

The proposed ITR sensor was envisioned to work with Inconel heat exchanger tubes, which have a diameter of about $d=0.875 "(2.225 \mathrm{~cm})$ and a wall thickness of $t=0.05 "(0.127 \mathrm{~cm})$. The conductivity of Inconel was taken to be $\sigma=8.2 \times 10^{5} \mathrm{~S} / \mathrm{m}$ in the calculations below.

Additionally, the relative permittivity and relative permeability were taken to be $\varepsilon_{\mathrm{r}}=1$ and $\mu_{\mathrm{r}}=1$, respectively. In SI units, the permittivity and permeability of free space are $\varepsilon_{0}=8.854 \times 10^{-12} \mathrm{~F} / \mathrm{m}$ and $\mu_{0}=4 \pi \times 10^{-7} \mathrm{H} / \mathrm{m}$, respectively. For the actual steam generator tubes, the frequency of operation would be selected such that the wall thickness is half a skin depth; that is, the frequency is chosen so that the depth of penetration equal to the two-way path through the wall. First, the propagation coefficient within the metallic wall is given by

$$
\gamma=\sqrt{j \omega \mu_{r} \mu_{o}\left(\sigma+j \omega \varepsilon_{r} \varepsilon_{o}\right)}=\alpha+j \beta
$$


The attenuation and phase coefficients are $\alpha$ and $\beta$, respectively. The depth of penetration, or skin depth, is given by the $1 / \mathrm{e}$ point. In a good conductor the conduction current is much greater than the displacement current so that we can write

$$
\gamma \approx \sqrt{j \omega \mu_{r} \mu_{o} \sigma}=\frac{(1+j)}{\sqrt{2}} \sqrt{\omega \mu_{r} \mu_{o} \sigma}=\alpha+j \beta
$$

Therefore the attenuation coefficient is

$$
\alpha \approx \sqrt{\frac{\omega \mu_{r} \mu_{o} \sigma}{2}}=\sqrt{f \mu_{r} \mu_{o} \sigma}
$$

This results in a skin depth of

$$
\delta_{s}=\frac{1}{\sqrt{f \mu_{r} \mu_{o} \sigma}}
$$

To achieve $\delta_{\mathrm{s}}=2 t$, we select the frequency to be $f=47.88 \mathrm{kHz}$ for Inconel tubes with $0.127 \mathrm{~cm}$ wall thickness.

To construct "antennas" to operate at this frequency as well as the necessary electronics to fit in such a restricted space would require specialized chip design(s). To circumvent this problem, a scaled version was proposed so that the electronics could be developed using discrete components. Based upon the size of the discrete electronic components, a scaled steam tube of nominal 3.5" $(8.89 \mathrm{~cm})$ diameter was proposed. To have the interior region of the tube be of the same electrical size, that is, size relative to wavelength, necessitated operation at a lower frequency, in this case, $\mathrm{f} \approx 12 \mathrm{kHz}$. The resulting wall thickness to achieve a two-way path equal to the skin depth at this frequency was $0.1 "(0.254 \mathrm{~cm})$. Thus, the sensor design began; the overall diameter (including a self-centering mechanism) was constrained to be the ID of the scaled tube. The diameter of the sensor (excluding the self-centering mechanism) was chosen to be $1.75 "(4.445 \mathrm{~cm})$.

A photograph of the prototype sensor is shown in Figure 2-1. The two major sections, transmit and receive are joined in the middle by a coupling nut. The protrusion on the left-hand end houses a fiber optic unit; it is recommended that fiber optics be eliminated from future versions of the sensor. 


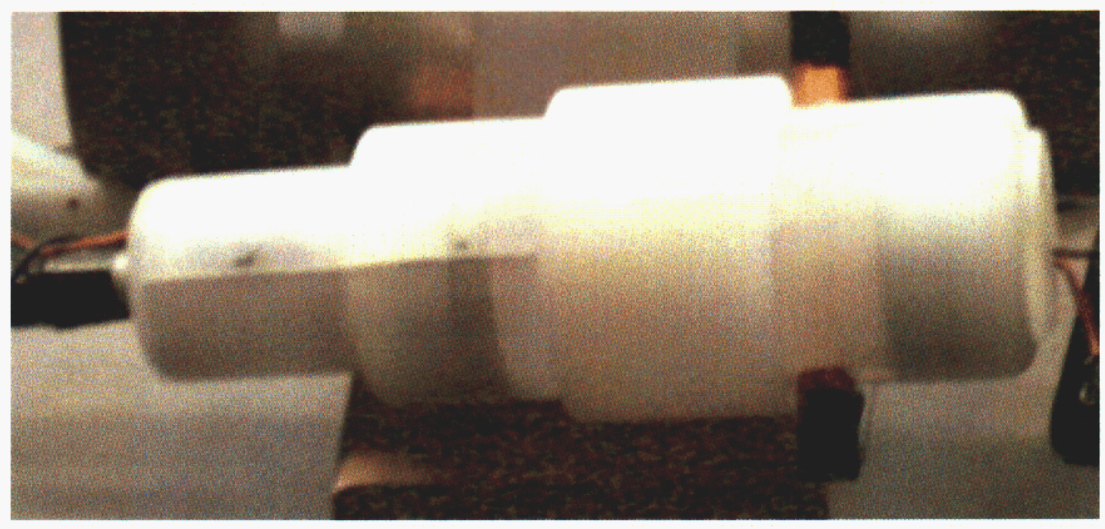

Figure 2-1. Photograph of Prototype ITR Sensor

A block diagram of the proposed ITR sensor is depicted in Figure 2-2. Starting in the upper lefthand corner, a computer with control/data acquisition board is depicted. The computer generates a digital waveform that is sent through a low-pass filter to produce a sinusoidal signal. The transmit signal is generated in the computer so that it is available as a reference during processing of the received signals. For transmission to the in-tube sensor, the signal is applied to a fiber optic transmitter. Within the sensor, a fiber optic receiver transforms back to the low-frequency electrical signal, which is then amplified. In the proposed sensor, a switch selected one of the two crossed loops to excite in sequence. The switch would be activated by a separate signal from the control board that is also sent to the in-tube sensor via fiber optic cable. The two transmit antennas are shown as coils in Figure 2-2.

The receive antenna was selected to be a monopole over ground, which was required to conveniently integrate the electronics. The ground plane provided some isolation of the receiver electronics; however, as discussed below, shielding was required to reduce system noise. The signal at the terminals of the receive antenna is then amplified, applied to a fiber optic transmitter, sent through a fiber to the optical receiver, and subsequently connected by wire to the data acquisition board.

Fiber optic cables were used to couple the test and control signals in to, and out of, the in-tube sensor. Since the actual sensor tubes as well as the scaled mockup operate well below cutoff from cylindrical waveguide modes at the frequencies required for penetration of the walls, it was originally thought that eliminating a second conductor, and hence the prospect of a TEM mode propagating longitudinally along the tube, was required. In practice it was found that metallic conductors forward and behind the sensor had little impact upon the received signals.

The block diagram for the prototype ITR sensor developed during this investigation is shown in Figure 2-3. It was based upon a single transmit antenna and thus required no switch or the associated control line. 


\section{ITR SCHEMATICS: MAIN BLOCK DIAGRAM}

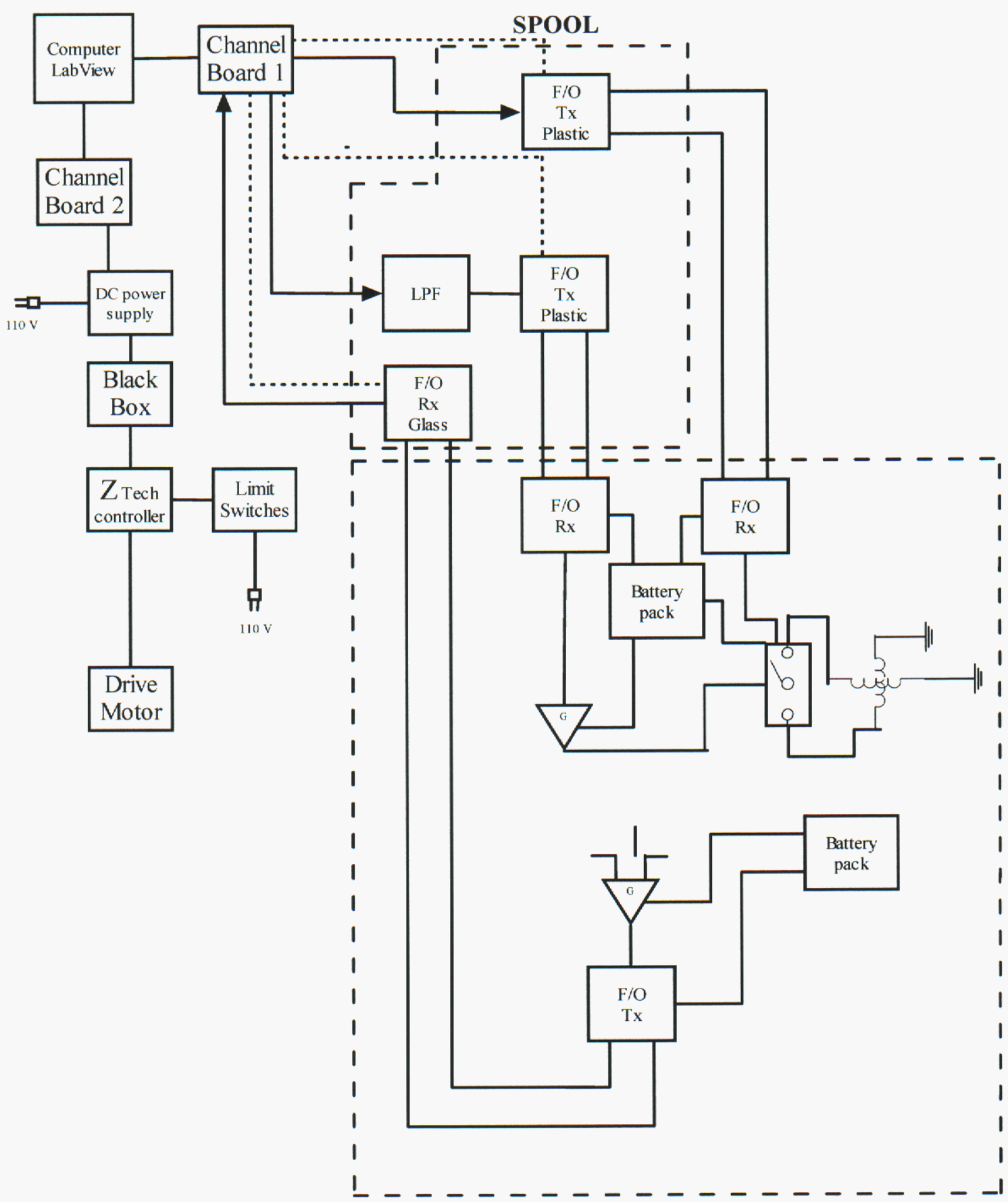

Figure 2-2. Proposed ITR Sensor Block Diagram 


\section{ITR SCHEMATICS}

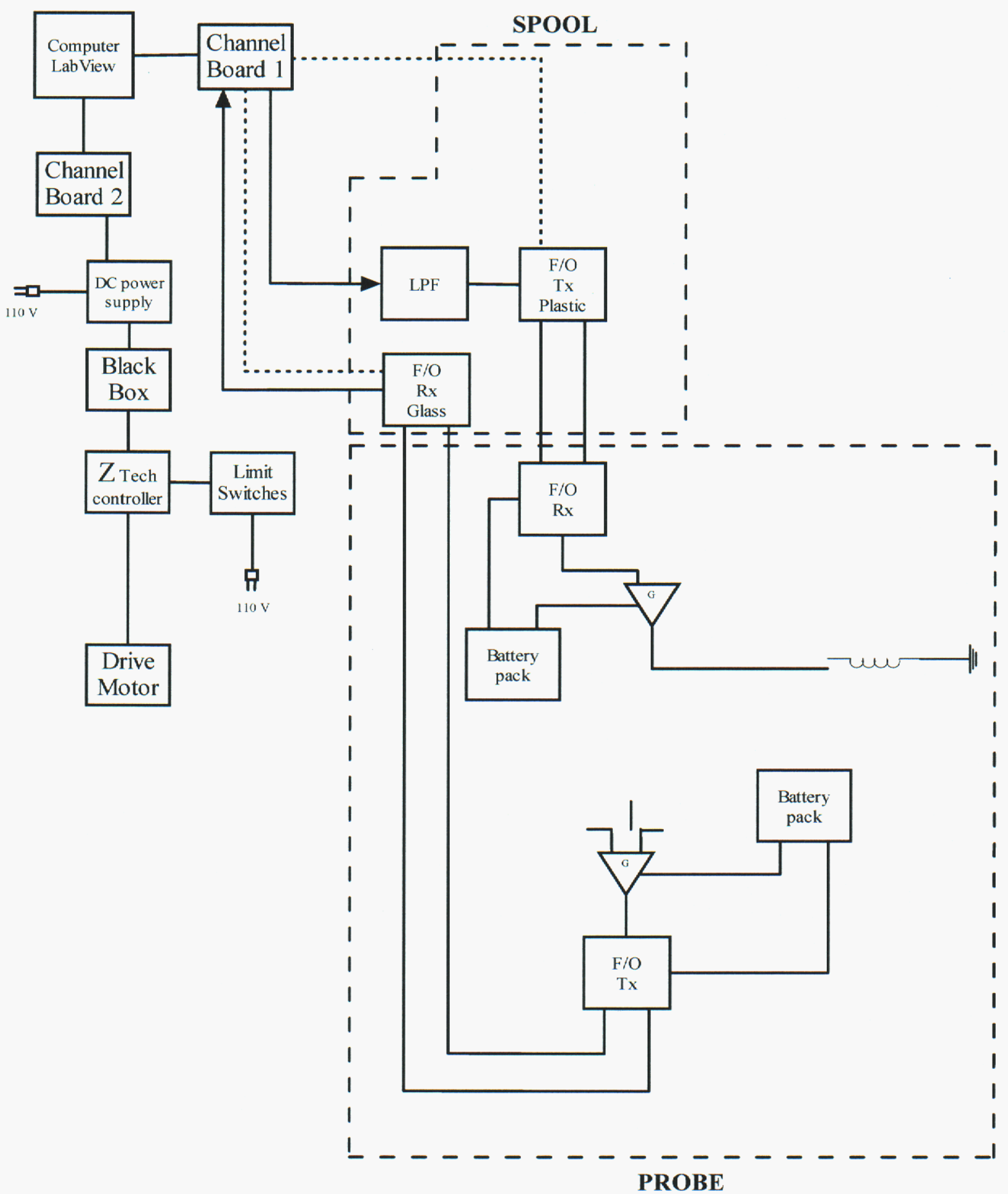

Figure 2-3. Block Diagram for the Prototype ITR Sensor

The next two subsections will document transmit and receive circuits as well as the associated electronics for the prototype sensor. 


\subsection{Signal Generation/Tx Circuit}

This section will focus upon the in-tube transmitter components. The digital generation of the transmit sine wave in the data acquisition/control board will be discussed in Section 3, this section will focus upon the path between the output of the board and the transmit antenna. We begin by considering the waveform at the output of the control board and then discuss the lowpass filtering required to achieve a sine wave from the quantized output.

The computer in conjunction with the data acquisition/control board generates a sampled version of a sine wave for transmission to the sensor itself. To eliminate the high frequency content that occurs using the discrete stair-step quantization of the sine wave, the output of the data acquisition/control board is low-pass filtered. Figure 2-4 shows the output of the board without filtering; Figure 2-5 that of the filtered response. The $3^{\text {rd }}$ order filter used to achieve this performance is shown in Figure 2-6.

$10 \mathrm{kHz}$ signal generated by computer 10 points per cycle Scan Rate $(\mathrm{Hz})$ : 200000.000 Column 1: Reference Signal

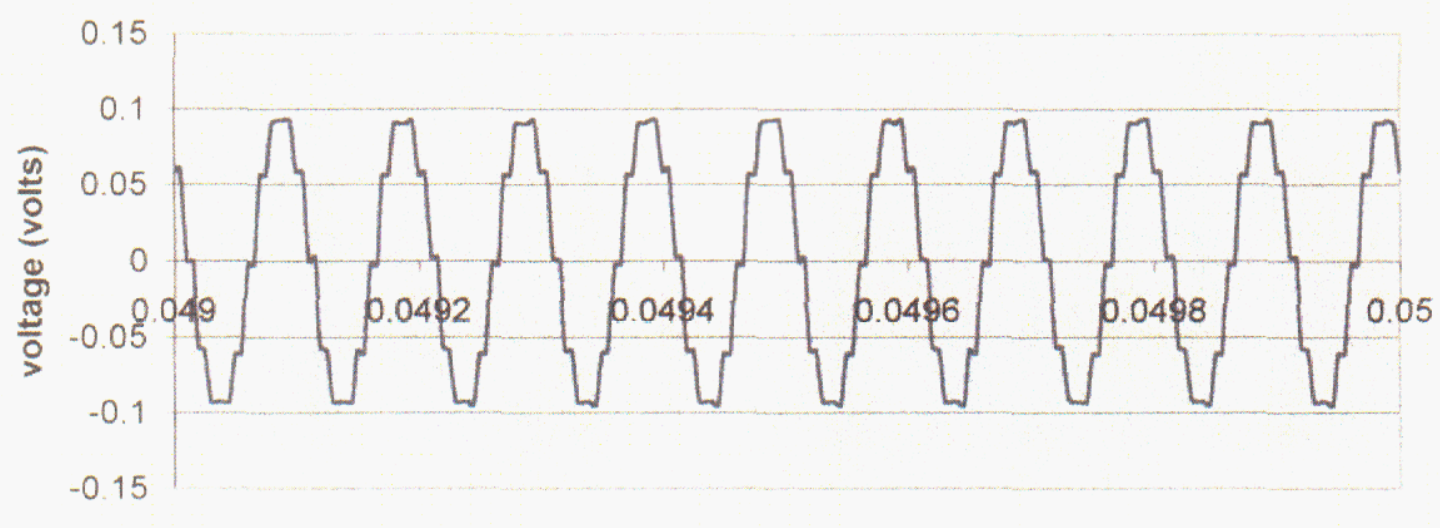

time (seconds)

Figure 2-4. Digitally generated Sine Wave 
$10 \mathrm{kHz}$ signal from filter 10 points per cycle Scan Rate $(\mathrm{Hz}): 200000.000$

Column 1: Reference Signal

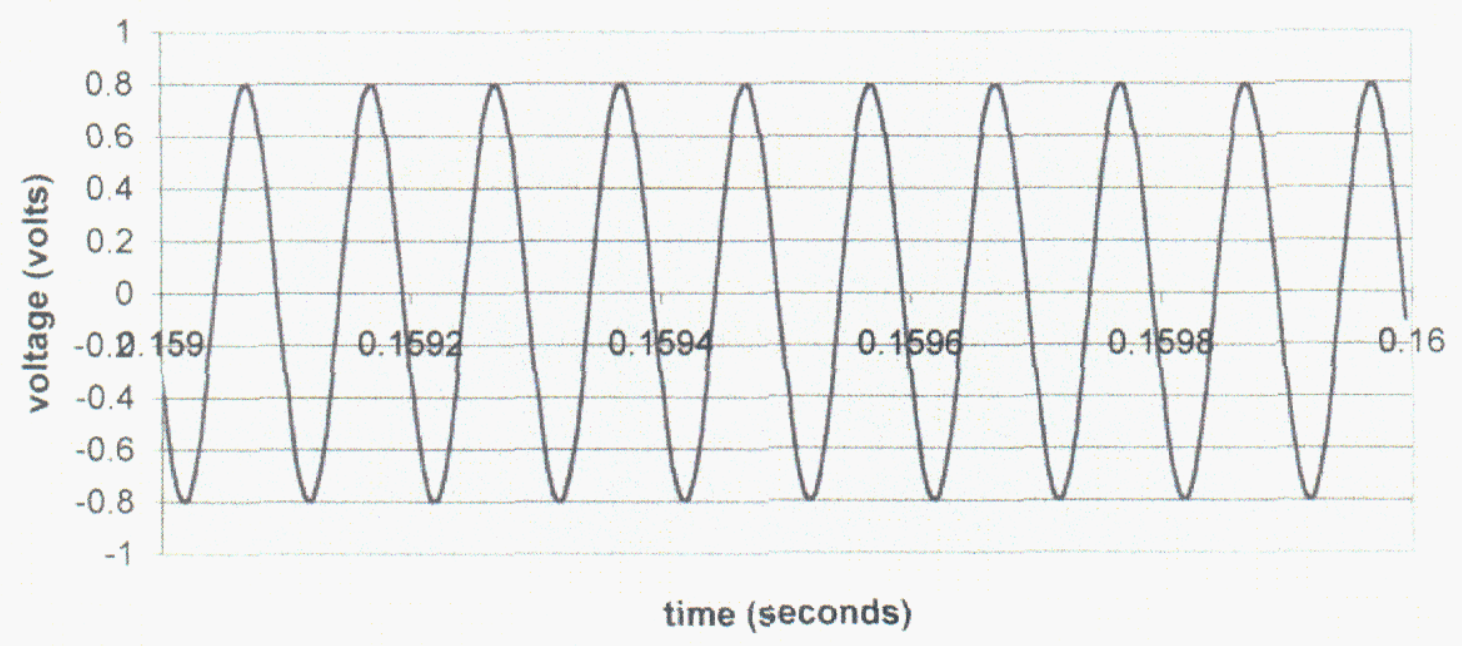

Figure 2-5. Digitally generated Sine Wave after Low-pass Filtering

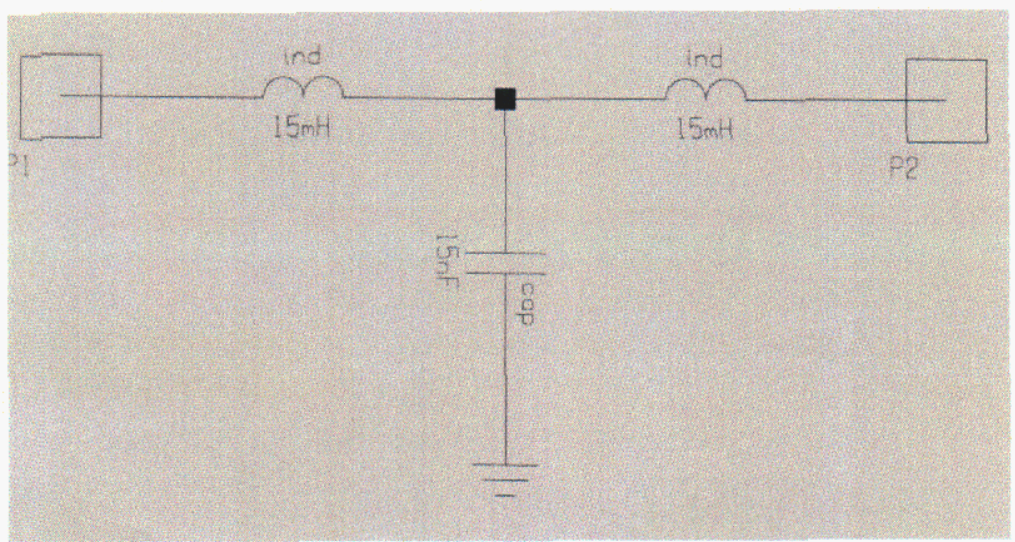

Figure 2-6. Low-pass Filter

The low-pass filter is constructed from two series inductors and a single shunt capacitor. The two inductors are $\mathrm{L}=15 \mathrm{mH}$ each, the shunt capacitor is $\mathrm{C}=15 \mathrm{nF}$. 
The output of the low-pass filter is applied to a plastic fiber optic transmitter, which converts electrical waveform into a corresponding optical signal. The circuit for the fiber optic transmitter is shown in Figure 2-7. The source of the optical signal is an emitting diode whose wavelength is 1300 nanometers. This allows the transmit waveform to be sent to the in-tube sensor without the need for metallic conductors (wires), that could perturb the electromagnetic fields as well as provide entry points for noise into the measurement system. For this link, a plastic fiber optic cable is used.

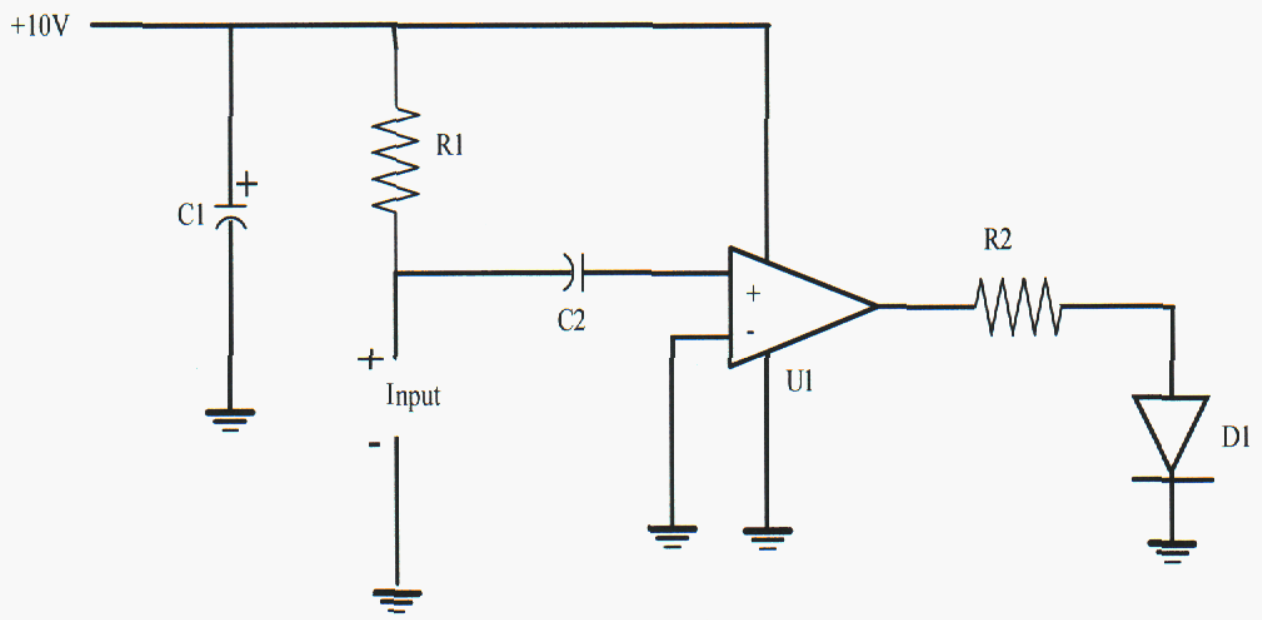

Figure 2-7. Plastic Fiber Optic Transmitter

Table 2-1. Plastic Fiber Optic Transmitter Parts List.

\begin{tabular}{|c|c|c|}
\hline Part & Description & Digi-Key* PN \\
\hline C1 & CAP Elect. 220UF 10V KS Radial & P964-ND \\
C2 & CAP 47000PF 10V Ceramic X\%R 0402 & PCC2142CT-ND \\
R1 & RES $22.1 \mathrm{~K}$ (ohms) $1 / 4 \mathrm{~W} 1 \% 1210 \mathrm{SMD}$ & P22.1KAACT-ND \\
R2 & RES 100(ohm) $1 / 4 \mathrm{~W} 1 \% 1210$ SMD & P100AACT-ND \\
U1 & IC AMP Audio Power ShutdownV8-SOIC & LM386M-1-ND \\
D1 & Photodiode Fiber/Optic Plastic & FB118 \\
\hline
\end{tabular}

* Digi-Key Corporation, Thief River Falls, Minnesota

Figure 2-8 shows the in-tube sensor electronics associated with the transmitter section. A primary difference between the ITR sensor and the eddy current method is that, in the former, crossed magnetic dipoles are situated about the centerline of the sensor, and therefore the tube, while in the latter, a coil is placed in closed vicinity to the wall of the steam generator tube. Thus, the ITR sensor, implemented with crossed antennas, would have the capability to sense the circumferential location of a defect whereas the eddy current method requires rotation of the sensor itself. In the prototype ITR sensor one of the magnetic dipoles (actually a ferrite-loaded loop or coil) is not implemented. To simulate the response of a double antenna system, data was simply taken in two orthogonal orientations. Again, it should be emphasized that rotation of the ITR sensor would not be required in the final design. 


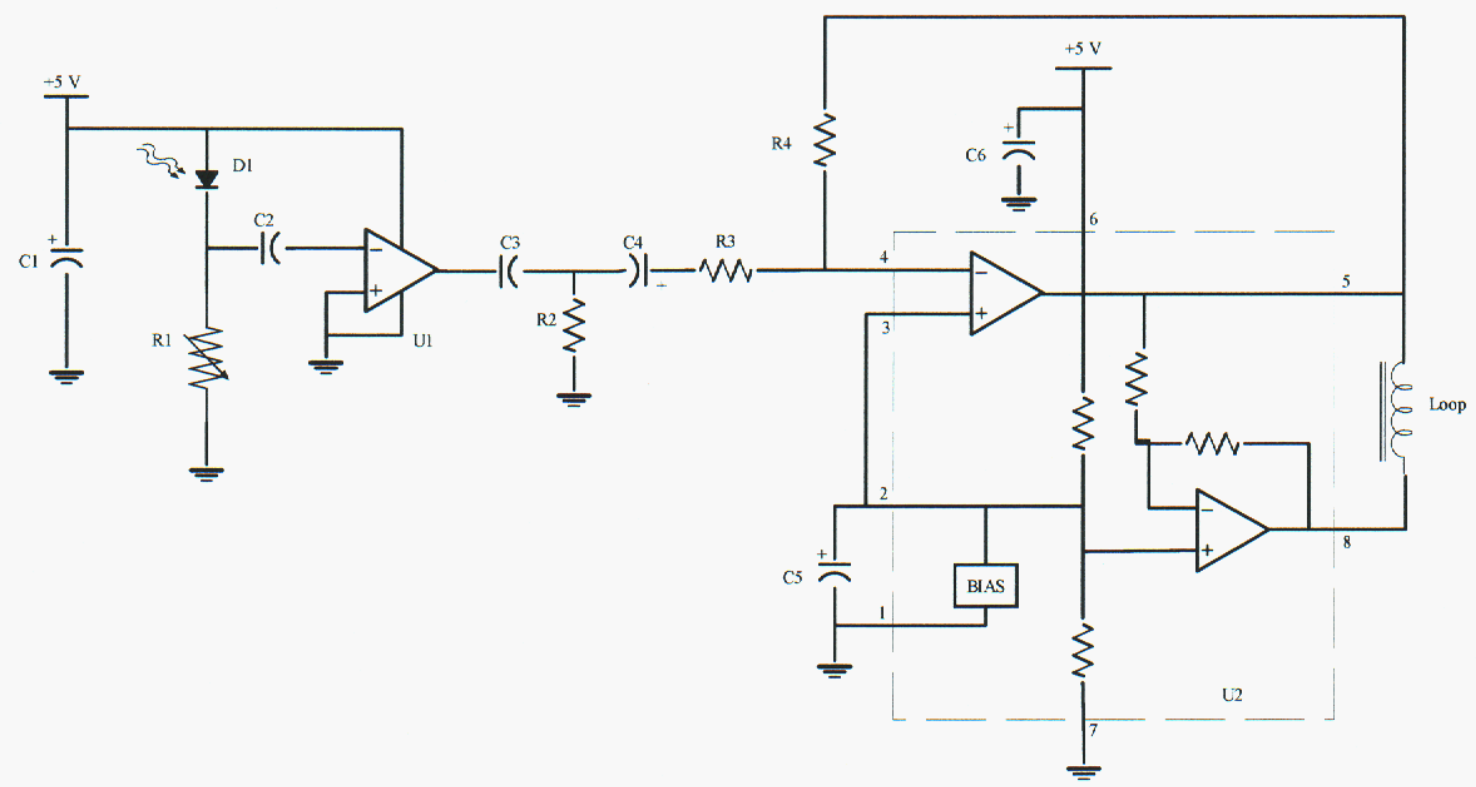

Figure 2-8. Transmitter Electronics and Antenna

Referring to Figure 2-8, the optical signal received and converted to the low-frequency sinusoid by diode D1 is subsequently amplified and applied to the transmit antenna. The amplifier, U1, at the output of the diode, D1, has a gain of about $G=100$. The signal is then applied to the input of the transmit amplifier, U2, through coupling capacitors. The gain of the transmit amplifier is set to be $\mathrm{G}=4.5$. The electronic chips and discrete components were integrated onto a doublesided PC board that fit within the inside diameter of the in-tube sensor housing. Grooves were machined on the interior wall of the housing to accept and hold the PC board in place. The coil antenna was wound with 120 turns of 30 -gauge wire on a ferrite rod 0.25 " in diameter (trimmed to a length 1.7"). The relative permeability of the ferrite rod used was $\mu_{\mathrm{r}}=125$. As the radiation resistance of the transmit antennas was negligible and the ohmic losses were not very large, the "antennas" presented primarily an inductive load on the output of the transmit amplifier.

Amplifier U2 was configured to drive this highly inductive load. A parts list for the in-tube transmitter components are shown in Table 2-2. 
Table 2-2. Transmitter Electronic Components

\begin{tabular}{|c|c|c|}
\hline Part & Description & Digi-Key*Part Number \\
\hline $\mathrm{C} 1$ & $\begin{array}{l}\text { CAP Elect 220UF } \\
10 \text { V KS Radial }\end{array}$ & P964-ND \\
\hline $2, \mathrm{C} 3$ & $\begin{array}{l}\text { CAP 47000PF 10V } \\
\text { Ceramic X5R 0402 } \\
\end{array}$ & PCC2142CT-ND \\
\hline $\mathrm{C} 4$ & $\begin{array}{l}\text { CAP .47UF 25V } \\
\text { Tant Te Series }\end{array}$ & PCS5474CT-ND \\
\hline $\mathrm{C} 5, \mathrm{C} 6$ & $\begin{array}{l}\text { CAP 1.0UF } 16 \mathrm{~V} \\
\text { Tant Te Series } \\
\end{array}$ & PCS3105CT-ND \\
\hline $\mathrm{D} 1$ & $\begin{array}{l}\text { Photodiode Fiber } \\
\text { Optic Plastic }\end{array}$ & FB120-ND \\
\hline $\mathrm{R} 1$ & $\begin{array}{l}\text { TRIM POT } 12 \\
\text { Turnn SMT } 5 \mathrm{~K} \Omega\end{array}$ & SM4W502-ND \\
\hline R2 & $\begin{array}{l}\text { RES } 100 \Omega 1 / 4 \mathrm{~W} \\
1 \% 1210 \mathrm{SMD}\end{array}$ & P100AACT-ND \\
\hline R3 & $\begin{array}{l}\text { RES } 22.1 \mathrm{~K} \Omega 1 / 4 \mathrm{~W} \\
1 \% 1210 \mathrm{SMD}\end{array}$ & P22.1KAACT-ND \\
\hline $\mathrm{R} 4$ & $\begin{array}{l}\text { RES } 100 \mathrm{~K} \Omega 1 / 4 \mathrm{~W} \\
1 \% 1210 \mathrm{SMD} \\
\end{array}$ & P100KAACT-ND \\
\hline U1 & $\begin{array}{l}\text { IC AMP Audio } \\
\text { Power Low V 8- } \\
\text { SOIC }\end{array}$ & LM386M-1-ND \\
\hline $\mathrm{U} 2$ & $\begin{array}{l}\text { IC AMP Audio } \\
\text { Power Shutdown 8- } \\
\text { SOIC }\end{array}$ & LM4871M-ND \\
\hline Loop & Ferrite Rod \& Wire & Amidon R61-025-400 \\
\hline
\end{tabular}

It should be noted that the original prototype sensor was designed to operate from within a dielectric housing; this was done to minimize the impact upon the fields produced by the transmit coil. However, due to coupling between the transmitter and receiver circuits other than through the antennas, that is, between PC board traces, component leads and wires. Both circuits were shielded in the final prototype sensor design. 


\subsection{Signal Reception/Rx Circuit}

As stated previously, a primary difference between the proposed ITR sensor and the eddy current method is that in the former, the reception of signals is accomplished using an electric antenna. This is a key feature of the proposed method; the defects on, or within, the tube wall, are sensed through observation of the axial electric field - by changing the excitation of the system using one of the two crossed magnetic dipoles at a time, the circumferential location of a defect can be determined, regardless of the orientation of the probe. The sense mechanism does not have to be in close vicinity to the wall as in the eddy current technique. The proposed ITR antenna was a dipole; however, space and isolation requirements forced this to be a monopole over ground in the prototype sensor, this antenna is located along the axis of the sensor, and therefore the tube, to measure the axial electric field. The receive electronics are shown in Figure 2-9.

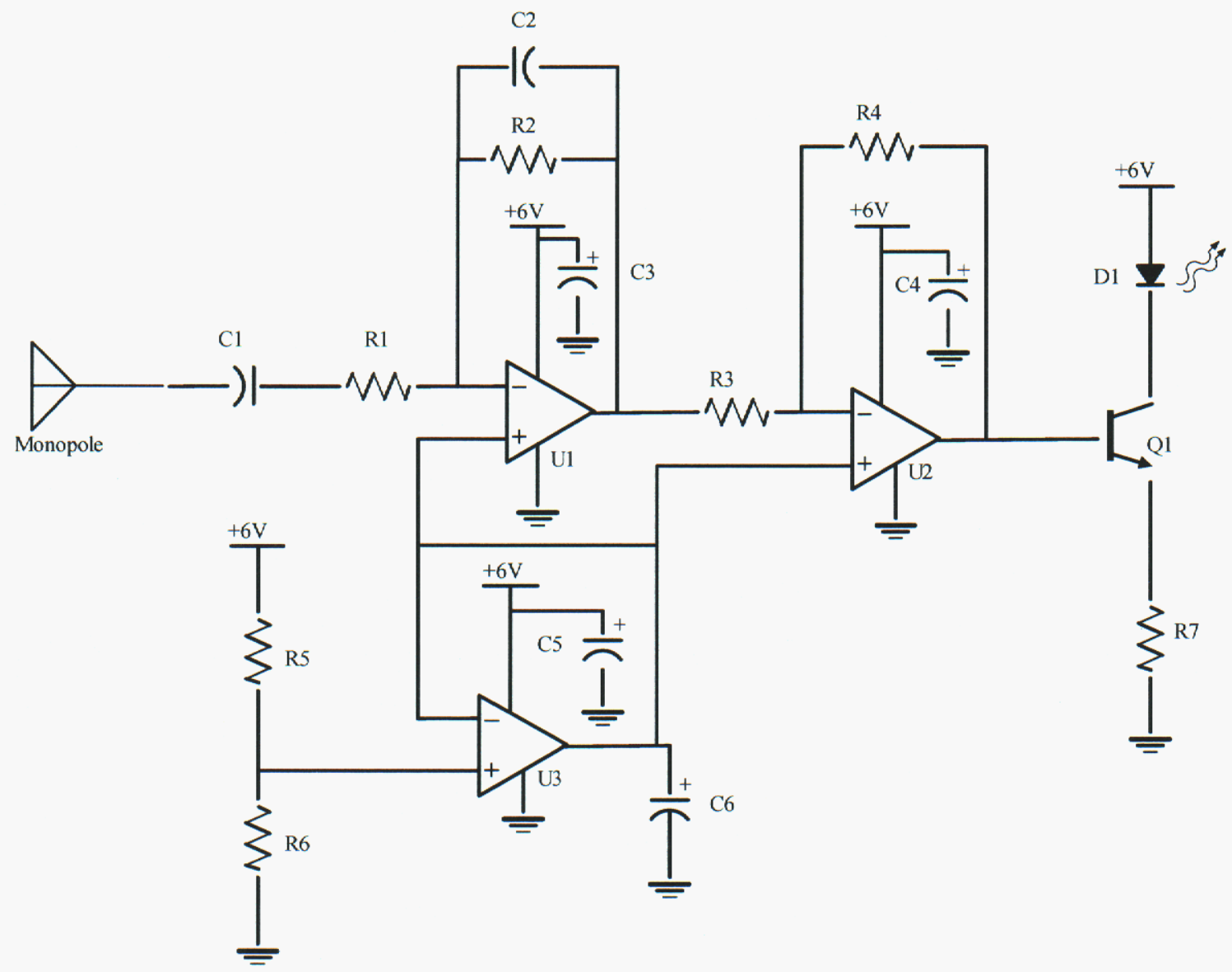

Figure 2-9 Receiver Electronics and Antenna

The parts list for the receiver components is shown in Table 2-3. 
Table 2-3. Receiver Electronic Components

\begin{tabular}{|c|c|c|}
\hline Part & Description & Digi-Key* Part Number \\
\hline $\mathrm{Cl}$ & $\begin{array}{c}0.47 \mathrm{uF} 25 \mathrm{~V} \\
\text { Tant TE Series }\end{array}$ & PCS5474Ct-ND \\
\hline $\mathrm{C} 2$ & $\begin{array}{c}\text { CAP Ceramic } \\
100 \mathrm{pF} 50 \mathrm{~V} 0603 \\
\text { SMD }\end{array}$ & PCC101ACVCT-ND \\
\hline $\mathrm{C} 3, \mathrm{C} 4, \mathrm{C} 5, \mathrm{C} 6$ & $\begin{array}{l}\text { CAP } 10 \mathrm{UF} 10 \mathrm{~V} \\
\text { Tant TE Series }\end{array}$ & PCS2106CT-ND \\
\hline D1 & $\begin{array}{c}\text { Transmitter LED St } \\
\text { Board Mt. }\end{array}$ & A20209-ND \\
\hline Q1 & $\begin{array}{l}\text { NPN SS GP Amp } \\
\text { Transistor TO92 }\end{array}$ & 2N3903-ND \\
\hline $\mathrm{R} 1, \mathrm{R} 3$ & $\begin{array}{c}\text { RES } 10.0 \mathrm{k} \Omega 1 / 4 \mathrm{~W} \\
1 \% 1210 \mathrm{SMD}\end{array}$ & P10,0KAACT-ND \\
\hline $\mathrm{R} 5, \mathrm{R} 6$ & $\begin{array}{c}\text { RES } 100 \mathrm{k} \Omega 1 / 4 \mathrm{~W} \\
1 \% 1210 \mathrm{SMD}\end{array}$ & P100KAACT-ND \\
\hline $\mathrm{R} 2, \mathrm{R} 4$ & $\begin{array}{c}\text { RES } 750 \mathrm{k} \Omega 1 / 4 \mathrm{~W} \\
1 \% 1210 \mathrm{SMD}\end{array}$ & P750KAACT-ND \\
\hline R7 & $\begin{array}{l}\text { RES } 18 \Omega 1 / 4 \mathrm{~W} \\
1 \% 1210 \text { SMD }\end{array}$ & P18AACT-ND \\
\hline $\mathrm{U} 1, \mathrm{U} 2, \mathrm{U} 3$ & $\begin{array}{l}\text { IC Dual In/Output } \\
\text { Op Amp 8-MSOP }\end{array}$ & LMC6482IMMCT-ND \\
\hline Monopole & $0.9 "$ long, $0.036 " \mathrm{dia}$ & RG-142 \\
\hline
\end{tabular}

* Digi-Key Corporation, Thief River Falls, Minnesota

The signal from the receive monopole is $\mathrm{AC}$ coupled through a capacitor, $\mathrm{C} 1$, to the input of a cascade of two amplifiers, each of which has a gain of $G=75$. The output of the amplifier cascade is then applied to the fiber optic transmitter. Again, it should be noted that the original prototype sensor was designed to operate from within the dielectric housing. However, on the receive side it was particularly important to shield the electronics - in many cases there was more coupling to the circuit board than to the receive antenna. Once shielding enclosed the electronics, this was no longer a problem.

The monopole was constructed from RG-142 coaxial cable. The diameter of the center conductor was 0.036 " and its length was $0.9 "$ ". A circular ground plane was constructed from printed circuit board; copper was left on the backside. The diameter of the ground plane was 1.7". A 0.116 " diameter hole was drilled in the center of the ground plane, the braided shield was pulled back and the dielectric of the coaxial line was inserted through the hole. Once the shield was soldered to the ground plane, the dielectric was trimmed back even with the outer surface of the ground plane. To make the monopole more rigid, the stranded center conductor was tinned with solder.

Once the signal is sufficiently amplified, it is applied to the fiber optic transmitter, which is used with a glass fiber optic cable. The fiber optic receiver circuit utilized to convert this back to an electrical signal outside the tube is shown in Figure 2-10. The associated parts list is shown in Table 2-4. 


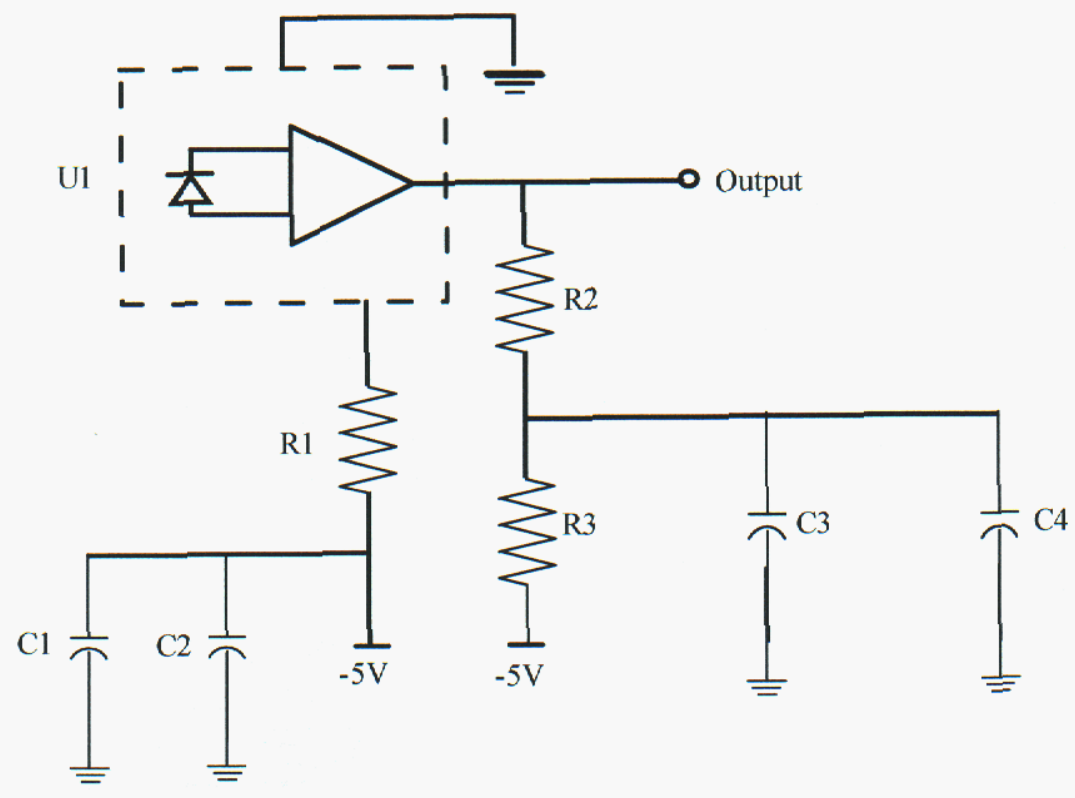

Figure 2-9. Glass Fiber Optic Receiver Circuit

Table 2-4. Glass Fiber Optic Receiver Circuit Parts List New Glass Fiber Optic Receiver

\begin{tabular}{|c|c|c|}
\hline Part & Description & Digi-Key* PN \\
\hline $\begin{array}{l}\mathrm{C} 1, \mathrm{C} 3 \\
\mathrm{C} 2, \mathrm{C} 4 \\
\end{array}$ & $\begin{array}{l}\text { CAP Ceramic } 100 \mathrm{pF}, 50 \mathrm{~V} 0603 \mathrm{SMD} \\
0.1 \mu \mathrm{F} 25 \mathrm{~V} \text { ceramic disc capacitor (p.516) }\end{array}$ & $\begin{array}{l}\text { PCC101ACVCT-ND } \\
\text { P4201-ND }\end{array}$ \\
\hline U1 & RECEIVER PREAMP ST BOARD MT & A20211-ND \\
\hline R1 & $10 \Omega$ & P10.0AACT-ND \\
\hline $\mathrm{R} 2, \mathrm{R} 3$ & RES $22.1 \mathrm{k}(\mathrm{Ohms}) 1 / 4 \mathrm{~W} 1 \% 1210 \mathrm{SMD}$ & P22.1KAACT-ND \\
\hline
\end{tabular}

*Digi-Key Corporation, Thief River Falls, Minnesota 
This page intentionally left blank 


\section{System Operation}

The experimental setup for the In Tube Radar (ITR) system is shown in Figure 3.1. The setup consists of a computer, probe, ZETEC pusher assembly, National Instruments NI 6711 analog output board, National Instruments PCI-MIO-16E-1 analog input/output board, two National Instruments SCB-68 connector blocks, probe pusher interface box, power supply, test tube, and safety switches. Two LabView ${ }^{\circledR}$ programs are used to control all aspects of communication between the probe and computer, control of the probe pusher, and power requirements to the various components. In this section of the report we will begin with an overview of the system operation, followed by a description of the LabView programs, and ending with a description of each component of the setup.

\subsection{Overview of System Operation}

The In Tube Radar (ITR) system is controlled and operated using two LabView programs, the probe positioning program and the data acquisition program. Here it is assumed that the probe is connected to the probe pusher feeder cable and is inside the test tube. Before the tube can be interrogated with the ITR probe, the probe must first be moved to one end of the tube or any desired location inside the tube. The task of positioning the probe within the tube is accomplished by the probe-positioning program.

Once the probe is at the desired location the user runs the data acquisition program. The data acquisition program records and stores the probe receiver antenna signal as the probe is pulled through the tube. A detailed discussion of the data acquisition program is provided below. After the probe stops, the process of repositioning the probe and running the data acquisition program can be repeated. Safety switches are utilized at each end of the tube to prevent the probe from being pushed or pulled out of the tube.

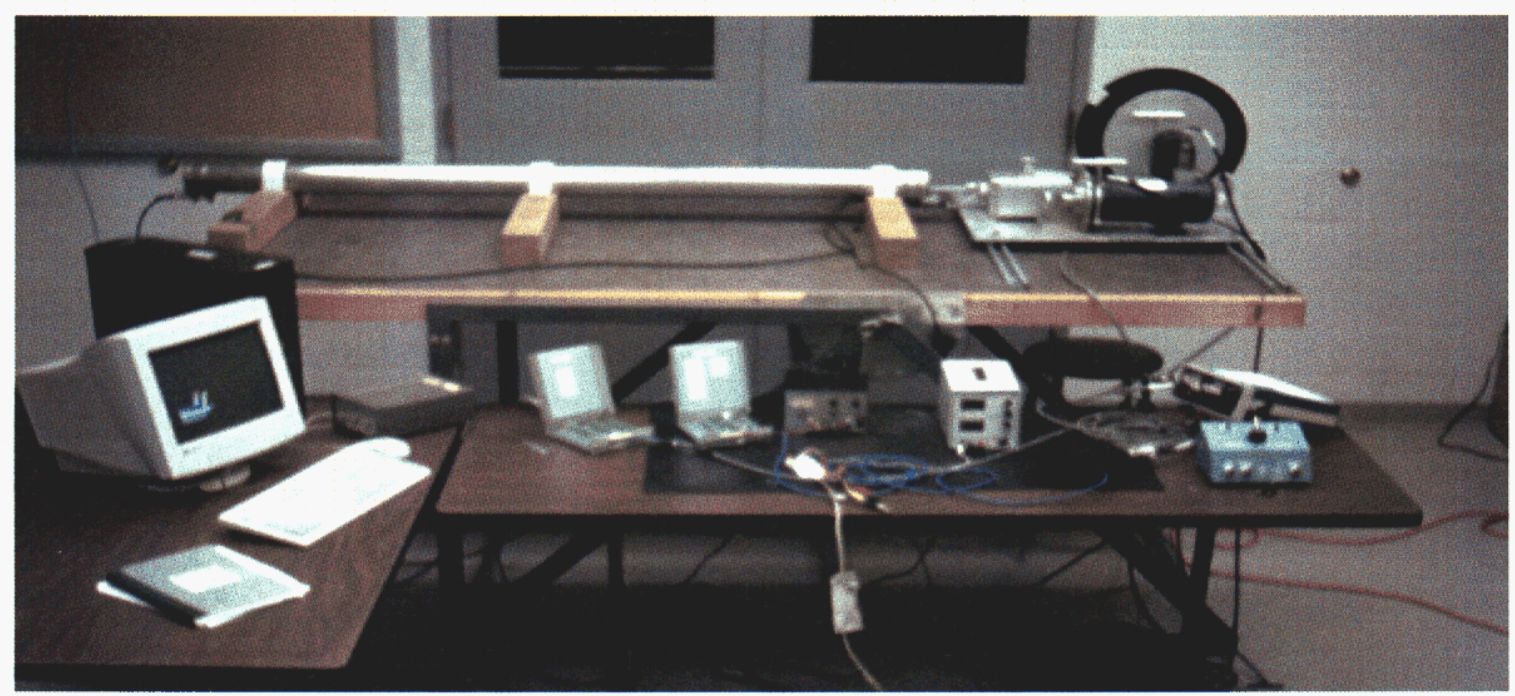

Figure 3-1. In Tube Radar System 


\subsection{Lab View Programs}

\section{Probe Positioning Program}

The probe-positioning program is used to position the probe inside the test tube. The program allows the probe to be moved continuously or in small increments (jog) in the forward or reverse directions. Figure 3.2 shows the user interface for the probe-positioning program. The speed at which the probe moves down the test tube is controlled by the sliding scale. Here the user simply adjusts the slider to the desired voltage level to achieve the desired speed at which the probe moves through the tube. Once the voltage is set the user depresses and holds the forward or reverse control buttons to push or pull the probe. Course movement of the probe is provided by the forward and reverse control buttons and fine movement is provided by the forward and reverse jog buttons. Figure 3.3 shows the wiring diagram for the probe-positioning program.

Control of the ZETEC probe pusher, using the probe positioning program, is accomplished with the PCI-MIO-16E-1 analog input/output board, SCB-68 connector block, \pm 15 Volt power supply, and the probe pusher interface box. The interface box (see Figure 3.8) contains an ISO122 isolation amplifier, which is used to eliminate a ground loop problem with the ZETEC probe pusher. The +15 and -15 Volt power supply signals are the supply power to the isolation amplifier. The PCI-MIO-16E-1 analog input/output board is installed in one of the PCI slots in the computer and is connected to the SCB-68 connector block. The voltage output channel from the PCI-MIO-16E-1 is connected to the input side of the isolation amplifier and the output signal from the isolation amplifier is the signal that controls the speed and direction of the probe pusher. $A+10$ Volt signal will push the probe at maximum speed and a -10 Volts signal will push the probe at maximum speed. Note that the isolation amplifier does not amplify the input signal and is used primarily to eliminate the ground loop problem. This system of components allows one to bypass the manual control box thus enabling control of the ZETEC probe pusher through LabView.

\section{Data Acquisition Program}

The data acquisition program performs the following: supplies power to optical transmitters and receivers, provides the signal to pull the probe through the test tube, provides the drive signal for the probe transmitter, and collects and stores data from the probe receiver antenna. Figure 3.4 shows the user interface for the data acquisition program and Figure 3.5 shows the wire diagram.

Before the program is run, the user enters the following quantities: frequency of the sine wave used to drive the probe transmitter, filename for the output data file, sample frequency, and sample time. All other values such as probe drive voltage, power requirements for optical components, drive signal amplitude, etc. are controlled within the wire diagram. The frequency of the sine wave is dependent on the tube material and thickness. The output data file contains the sample frequency and the sampled receiver antenna signal. This file can be opened at a later time to interrogate the receiver antenna signal. The sample frequency is the number of samples of the receiver antenna signal that are acquired per second. The sample time controls the length of time data is collected and the length of time for which the probe is pulled through the tub.

The function of the data acquisition program is accomplished with the components used by the probe positioning program, the NI 6711 analog output board, a second SCB-68 connector block, fiber optic components, and the probe. When the data acquisition program is run, it first supplies power to the fiber optic components external to the probe and outputs the probe pusher 


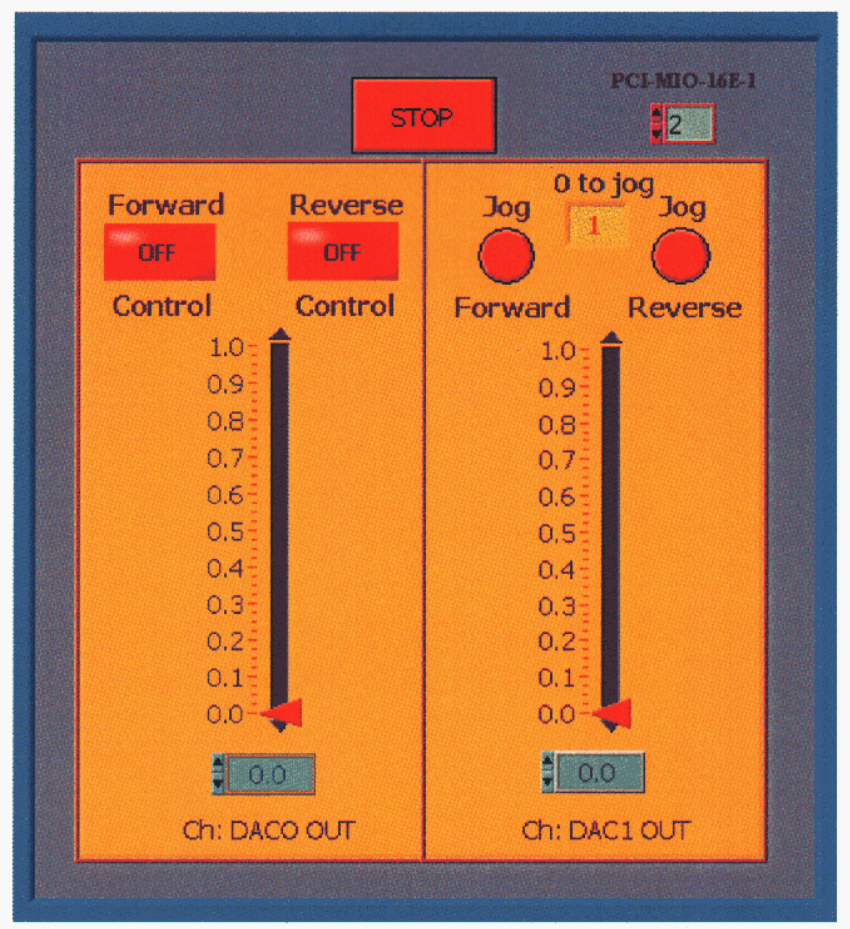

Figure 3-2. User Interface for Probe Positioning Program

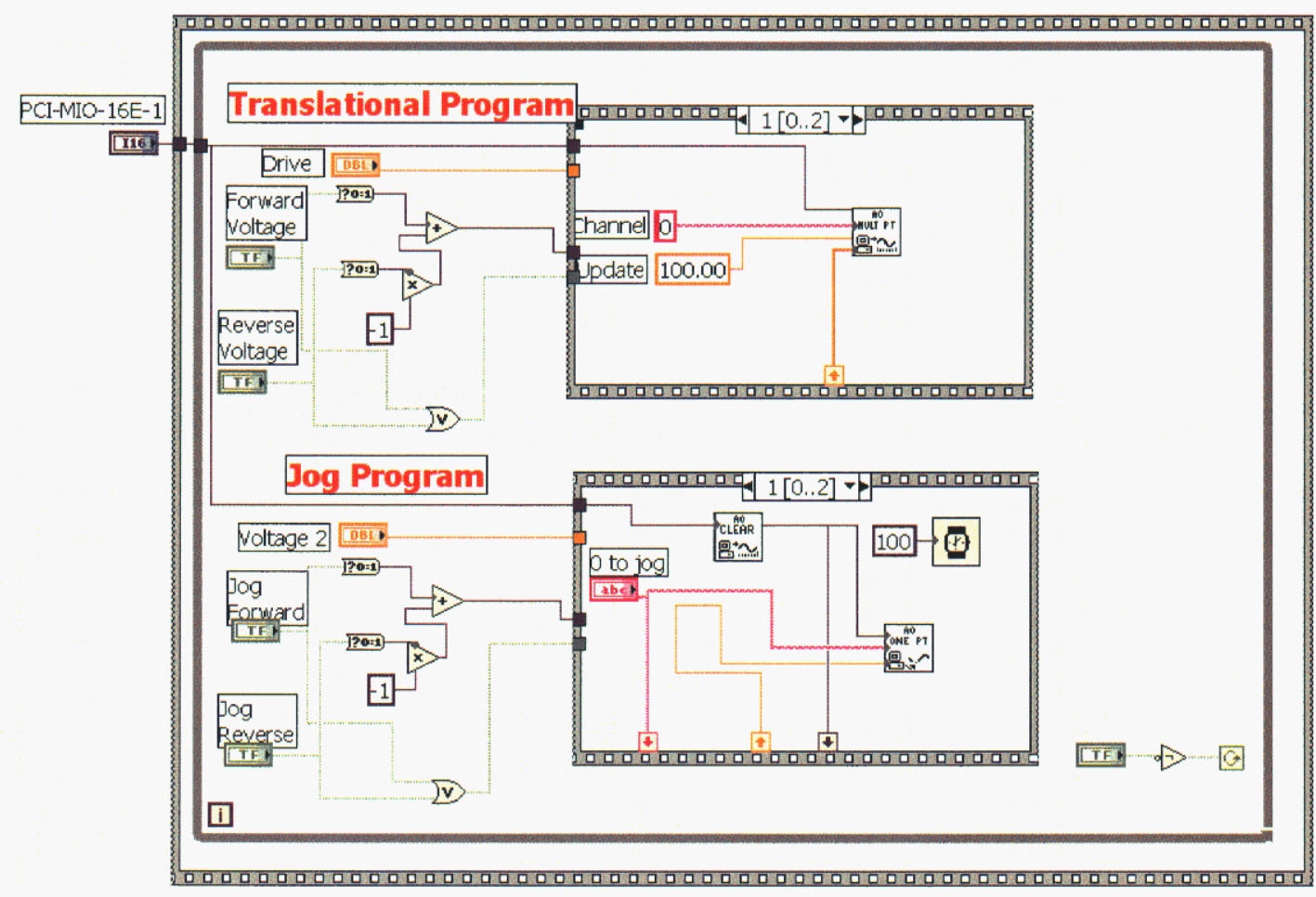

Figure 3-3. Wire Diagram for Probe Positioning Program 


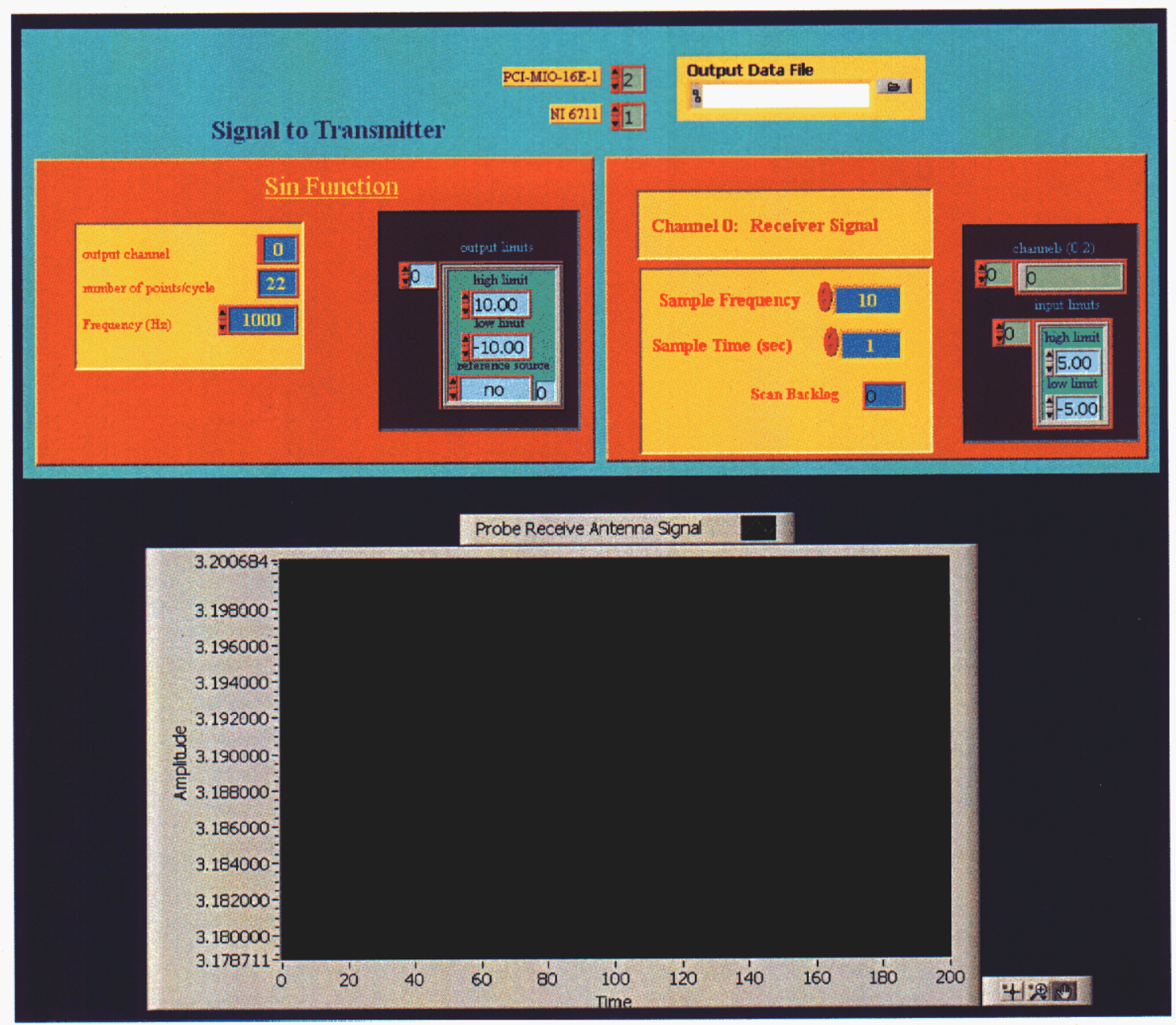

Figure 3-4. User Interface for Data Acquisition Program

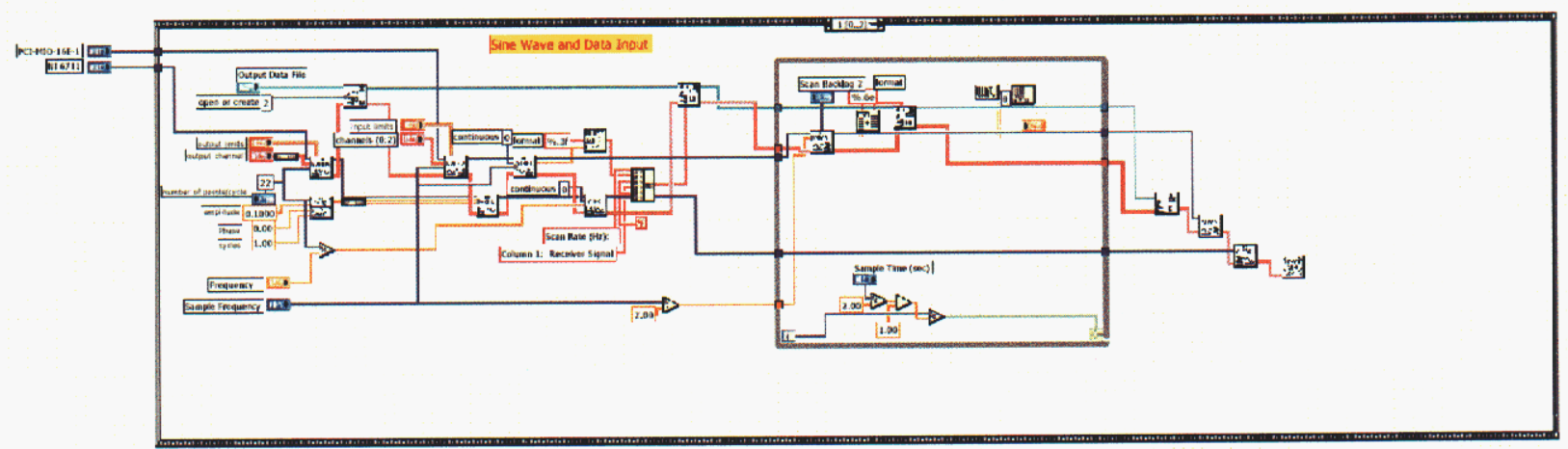

Figure 3-5. Wire Diagram for Data Acquisition Program

drive signal used to start pulling the probe. This is accomplished with the NI 6711 analog output board. Batteries located within the probe are used to supply power to the probe circuits. Next the output data file is opened and the sine wave is generate. Once the sine wave is generated it is converted to an analog signal and sent to a fiber optic transmitter. The fiber optic transmitter 
converts the electrical signal to an optical signal and transmits the signal via fiber optic cable to the probe. A fiber optic receiver on the probe converts the sine wave signal back to an electrical signal and then drives the probe transmitter with this signal. The probe receiver antenna signal is converted from an electrical signal to an optical signal. This signal is then transmitted via fiber optic cable to a fiber optic receiver. This is accomplished with a fiber optic transmitter located in the probe. The fiber optic receiver converts the optical signal to an electrical signal. This signal is then converted from an analog signal to a digital signal and is written to the output data file and is also displayed on the front panel display. The signal that is sent to the probe is a continuous signal. The signal from the probe receiver antenna is double buffered. This ensures that data points are not lost due to the speed at which data are written to a file. The process of reading the probe receiver antenna signal and writing it to a file is done in one second intervals and is controlled by a while loop (see Figure 3.5). The while loop keeps track of how many seconds of data has been written to the output file. Once the number of seconds of data written to the file is equal to the sample time that was entered on the front panel, the program exits the while loop, stops the probe, and resets all supply power voltages to zero. After the probe stops, the process of repositioning the probe and running the data acquisition program can be repeated.

\subsection{System Components}

\section{ZETEC Probe Pusher Assembly}

The ZETEC probe pusher assembly is shown in Figure 3.6. The main function of the probe pusher is to pull and push the probe through the test tube. The fiber optic plastic transmitter and circuit and the fiber optic glass receiver and circuit are housed inside the spool. A slip ring is used to ensure that the wiring to this circuit does not twist as the spool rotates.

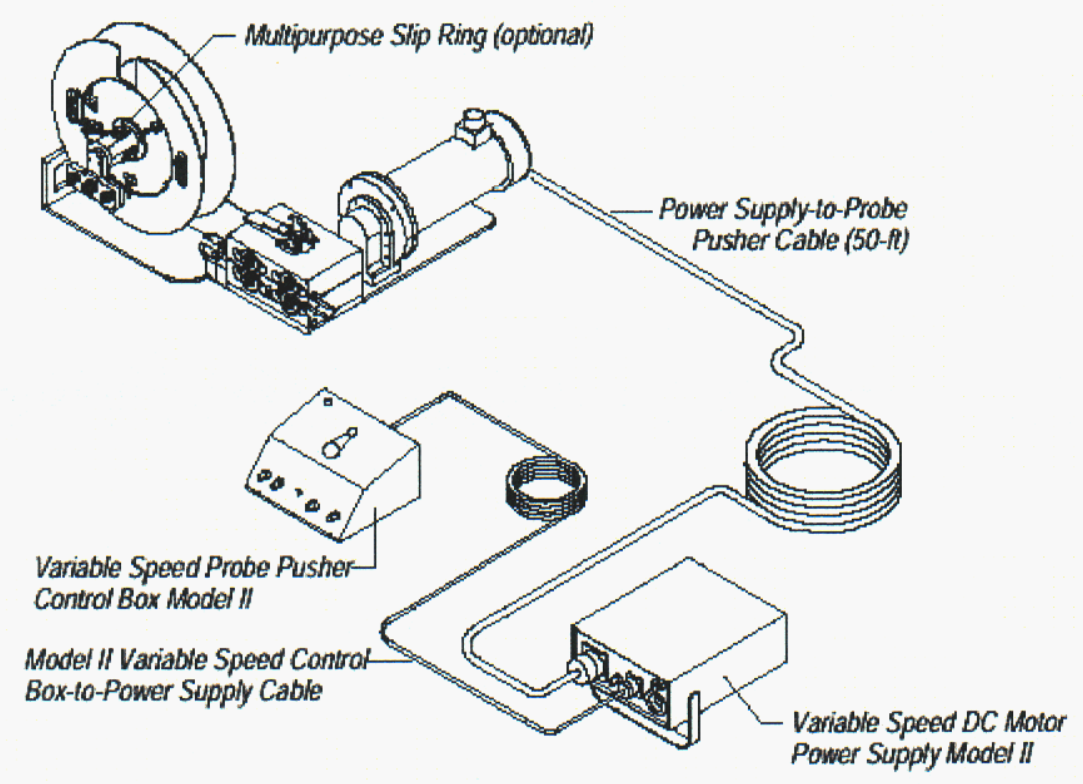

Figure 3-6 ZETEC Probe Pusher Assembly

\section{Power Supply}

The DC power supply (Figure 3.7 ) is used to provide $\mathrm{a}+15$ and -15 Volt signal to power the ISO122 isolation amplifier located in the probe pusher interface box. 

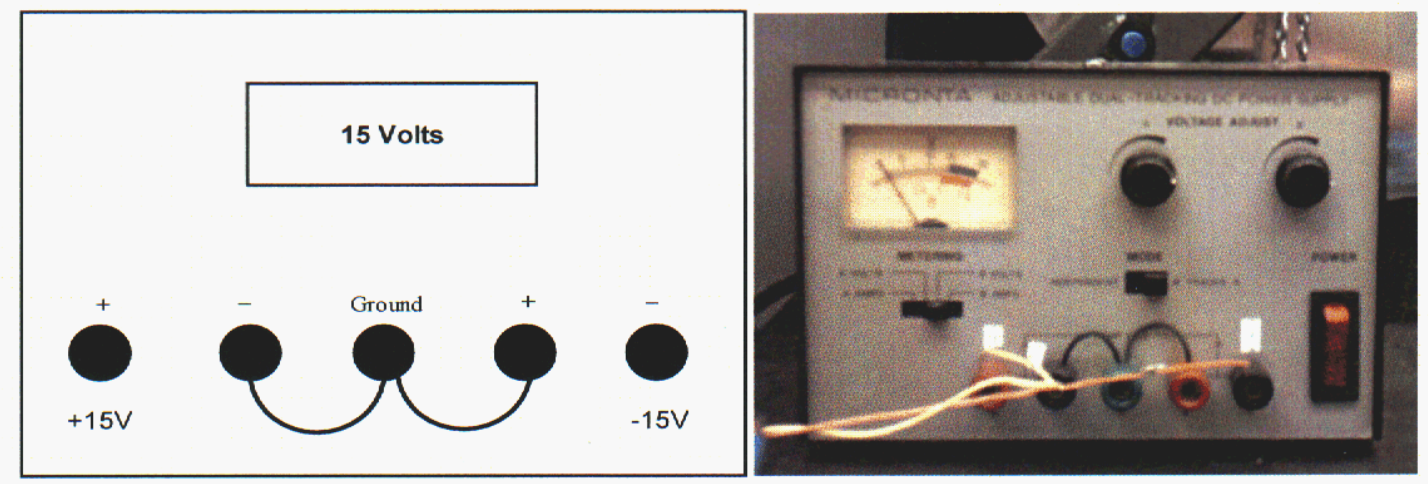

Figure 3-7. DC Power Supply

\section{Probe Pusher Interface Box}

The probe pusher interface box (Figure 3.8) contains an ISO122 isolation amplifier, which is used to eliminate a ground loop problem with the ZETEC probe pusher. The +15 and 15 Volt power supply signals from the DC power supply are the supply power to the isolation amplifier. The voltage output channel from the PCI-MIO-16E-1 is connected to the input side of the isolation amplifier and the output signal from the isolation amplifier is the signal that controls the speed and direction of the probe pusher. The probe pusher interface box allows one to bypass the manual control box thus enabling control of the ZETEC probe pusher through LabView.

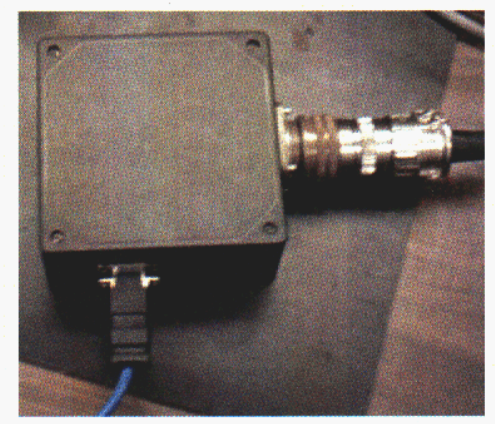

Figure 3-8. Probe Pusher Interface Box

\section{PCI-MIO-16E-1 Analog I/O Board}

The National Instruments PCI-MIO-16E-1 analog input/output board contains 8 differential analog input channels and 2 differential analog output channels. This board is capable of acquiring 1.5-mega samples/second and outputting 1 mega samples/second. Both input and output channels have a 12-bit resolution. The PCI-MIO-16E-1 board is installed in a PCI slot in the computer and is connected to a SCB- 68 connector block. All the electrical connects to the PCI-MIO-16E-1 board are accomplished through the SCB- 68 connector block.

The PCI-MIO-16E-1 board serves two functions: (1) to provide the analog signal needed to control the probe pusher (probe positioning program and data acquisition program) and (2) to acquire or sample data from the probe receiver antenna. This particular arrangement allows the probe receiver antenna signal to be sampled at $1.5 \mathrm{MHz}$. Figure 3.9 shows the SCB- 68 connector 
block along with the wiring. Note that only 4 wire are actually used in the finial arrangement, 2 wires for the input signal and 2 wires for the output signal. The two unconnected wires shown in the figure contain the input signal from the probe receiver antenna. The 2 wires that are connected contain the sine wave signal that is used to drive the probe transmitter. The sine wave signal was used to help write and fine-tune the data acquisition program. Figure 3.10 shows the wiring schematic for the PCI-MIO-16E-1 board.

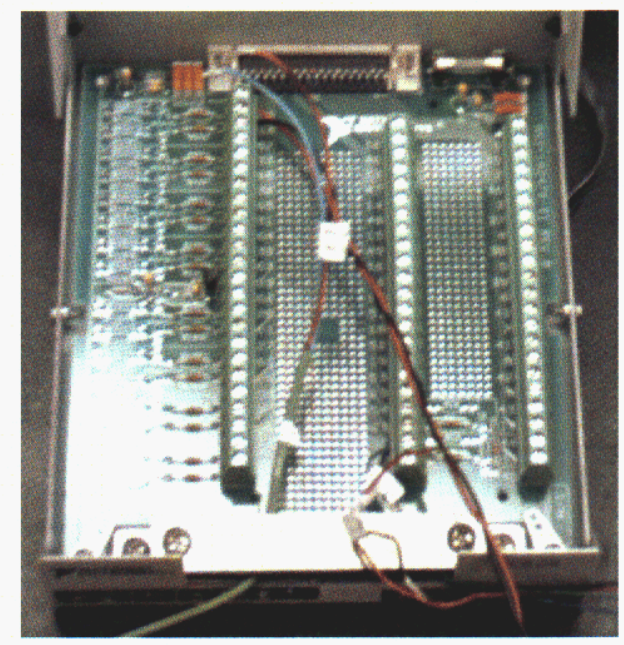

Figure 3-9. SCB-68 Connector Block for the PCI-MIO-16E-1 Board

\section{NI 6711 Analog Output Board}

The National Instruments NI 6711 analog output board contains 4 analog output channels. This board is capable of outputting $1 \mathrm{mega}$ samples/second/channel. The output channels have a 12bit resolution. The NI 6711 board is installed in a PCI slot in the computer and is connected to a SCB-68 connector block. All the electrical connects to the NI 6711 board are accomplished through the SCB-68 connector block.

The NI 6711 board serves 4 functions: (1) to provide the sine wave drive signal for the probe transmitter, (2) provide power to the optical transmitter (probe transmitter drive signal) located within the probe pusher spool, (3) provide power to the optical receiver (probe receiver antenna signal) located within the probe pusher spool, and (4) provide power to an amplifier used in the optical transmitter circuit. This particular arrangement allows the frequency of the drive signal to be on the order of $200 \mathrm{kHz}$ if needed. Figure 3.11 shows the SCB- 68 connector block along with the wiring and Figure 3.12 shows the wiring schematic for the NI 6711 board. 


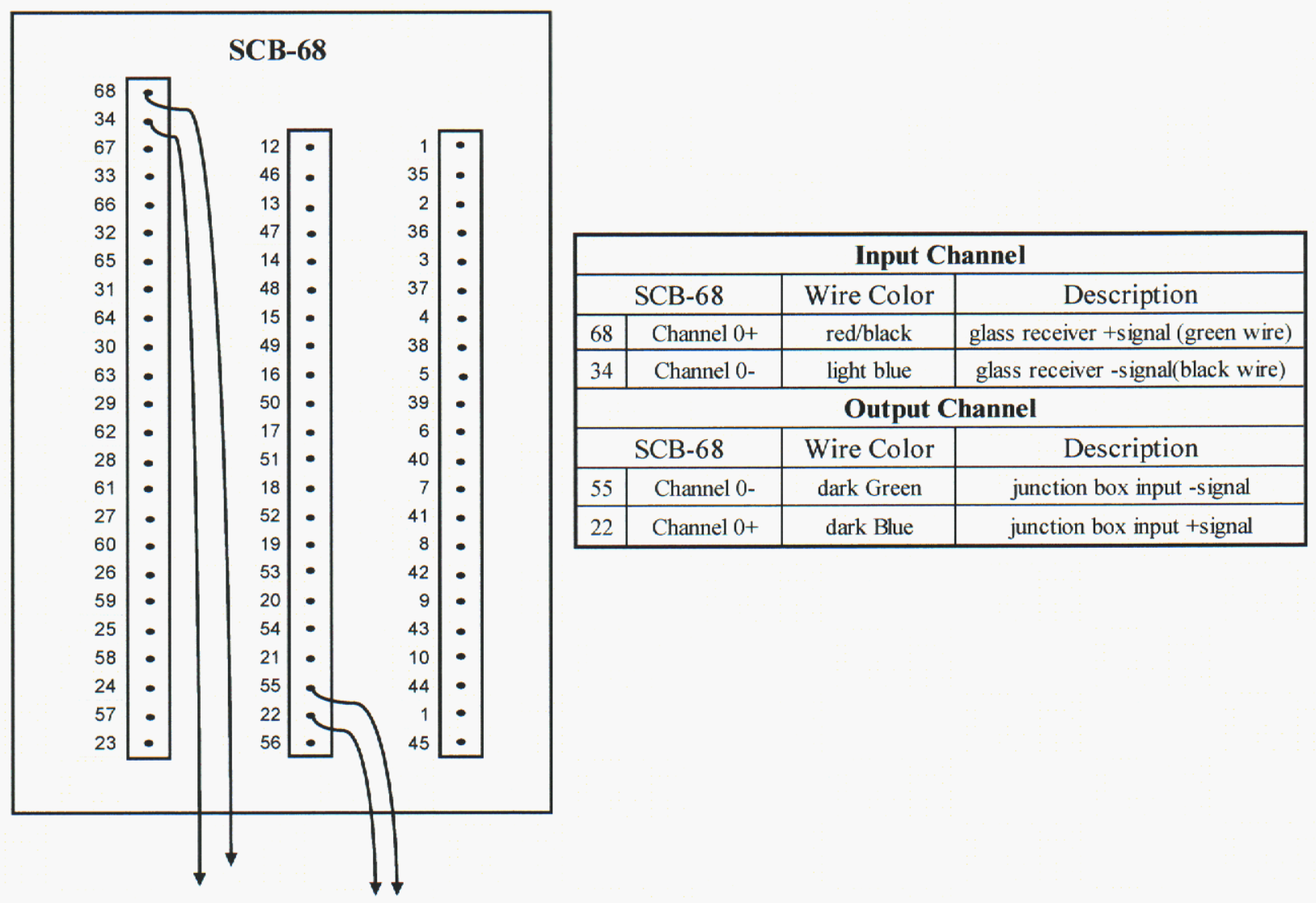

Figure 3-10. Schematic for PCI-MIO-16E-1 Board

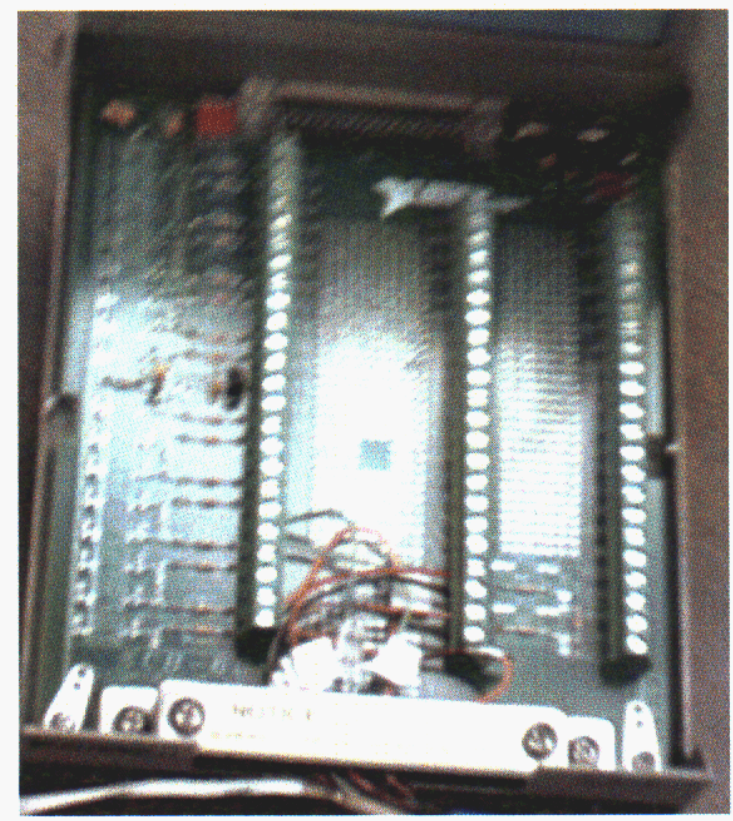


Figure 3-11. SCB-68 Connector Block for the NI 6711 Board

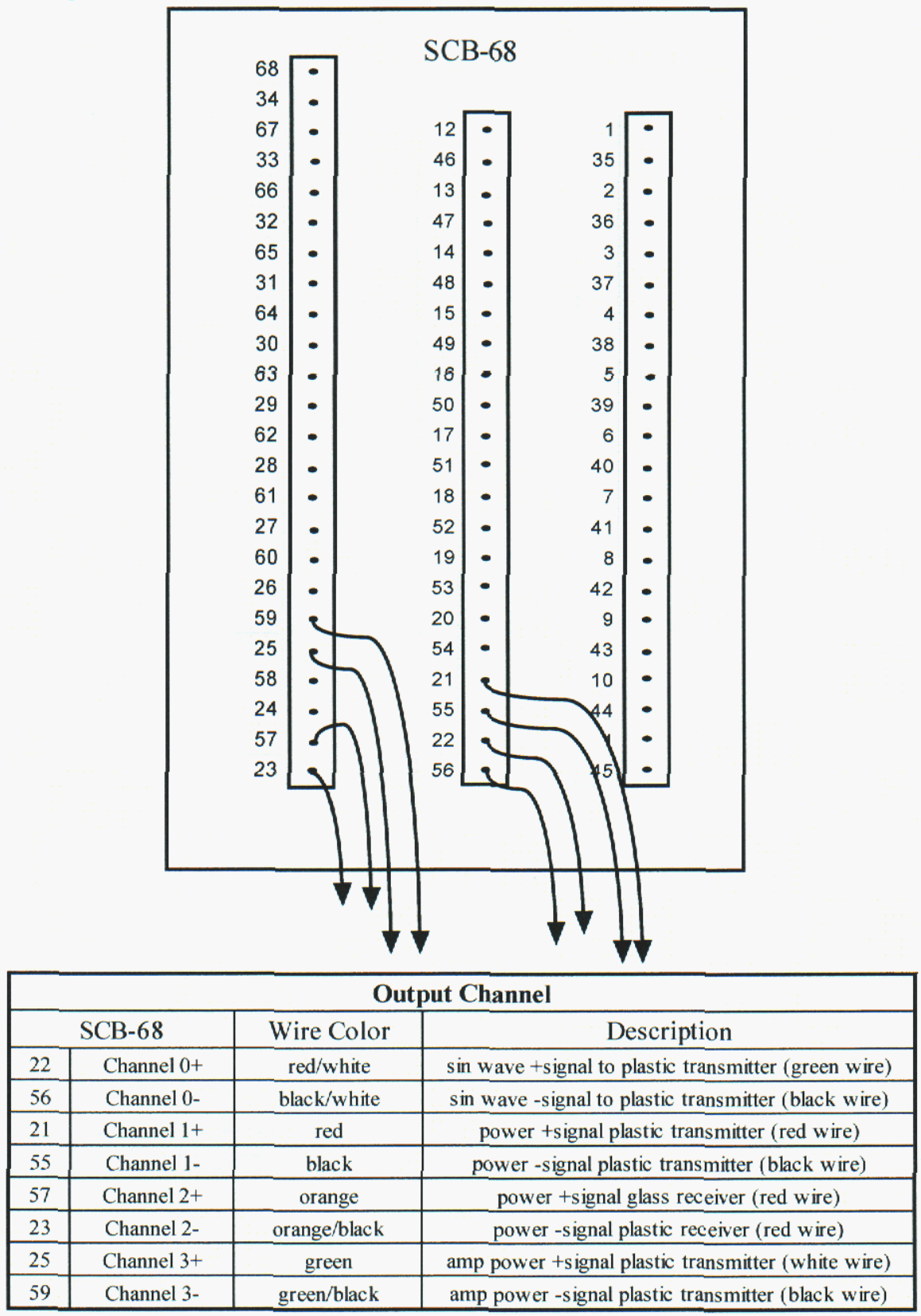

Figure 3-12. Schematic for NI 6711 Board

\section{Safety Switch Assembly}

The safety switch assembly is a cutoff device that protects the probe from damage in the event of a drive system malfunction. Limit switches prevent the probe from moving past the switches located at the ends of the tube. Without these switches, the probe could be pulled into the ZETEC pusher assembly or pushed out of the end of the tube, possibly damaging the probe. Figure 3.13 shows one of the safety switches mounted on the test tube. 


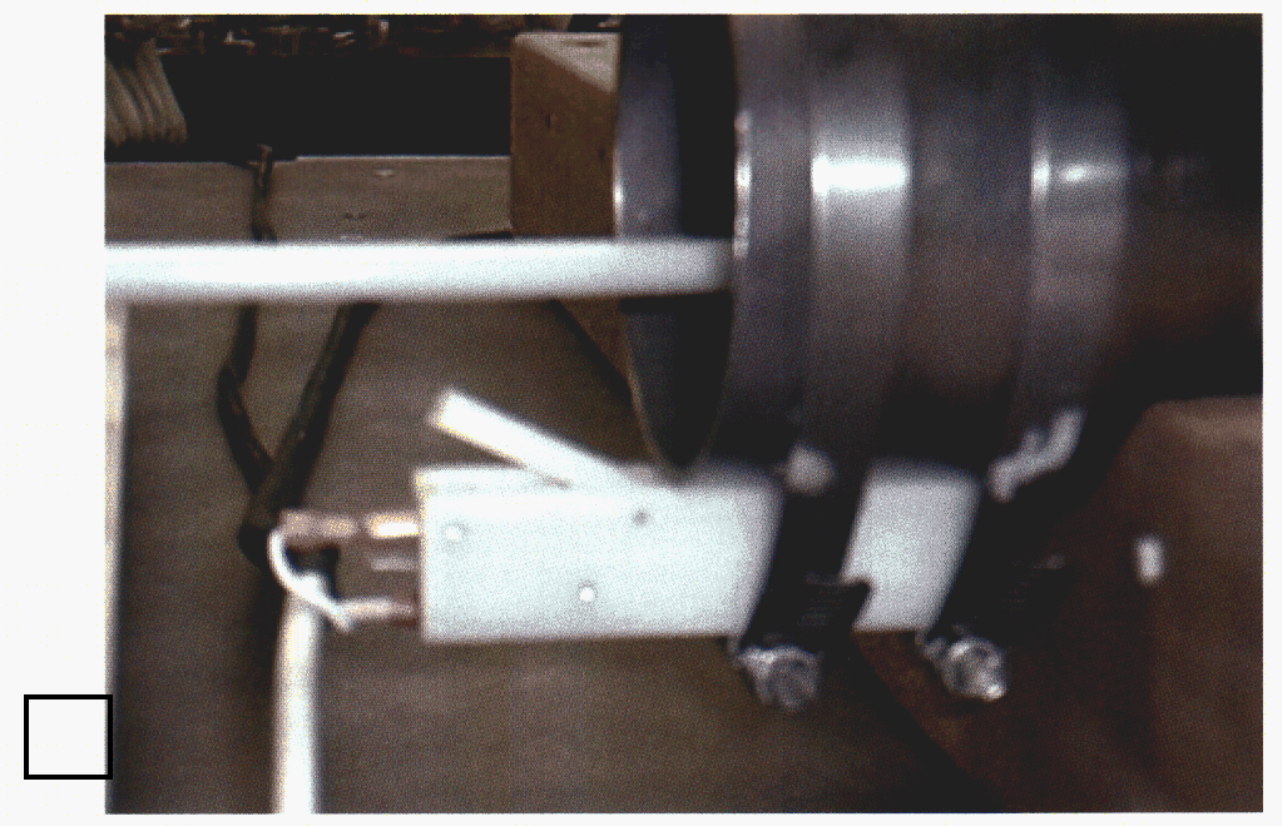

Figure 3-13 Safety Switch

\section{Probe Assembly}

Figure 3.14 shows the probe that is used to interrogate the test tube. The probe transmitter is driven by a sine wave and data are collected from the probe receiver antenna. The probe is described in sections 2 and 5 of this report.

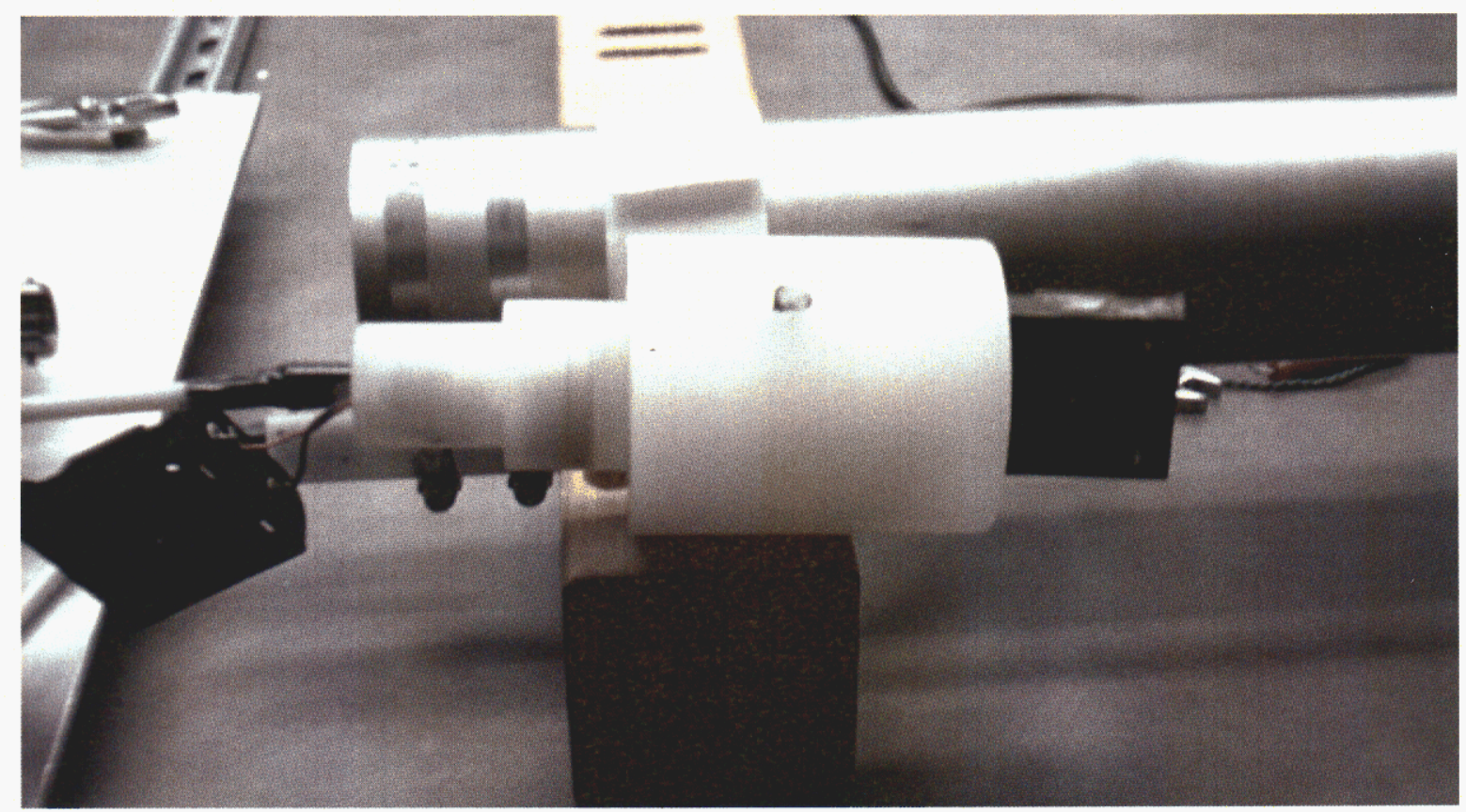

Figure 3-14 Probe Assembly 


\section{Measurements}

The development of the prototype ITR sensor was accomplished in steps. That is, each circuit subsystem was developed independently and then integrated into the system. To quantify the performance of the transmit $(\mathrm{Tx})$ and receive $(\mathrm{Rx})$ antennas, bench-top tests were run using standard laboratory instrumentation prior to integration with the electronics. Once the antenna configurations were complete it was then possible to complete the design of the amplifiers, etc. Once the prototype sensor was fabricated, tests were run in different tubes with varying defects. This chapter is split into two main sections: 1) out-of-tube testing of antennas and 2) in-tube testing. The former was done using a function generator to excite the transmit antenna; the signal at the terminals of the receive antenna was measured using an oscilloscope. In the latter, the function generator substituted for the digitally generated signal but the computer was used for data acquisition.

\subsection{Out-of-Tube Testing}

Tests of the coupling between antennas were done to study the performance of both transmit and receive units. The first test completed was the coupling between an air-loaded transmit coil and a monopole receive antenna. The former was approximately 120 turns of 30 -gauge wire wound around a non-magnetic core. For convenience a wooden dowel was used. The resulting transmit coil was 1.7 " long and 0.25 " in diameter. The monopole was 0.9 " long, the wire diameter was 0.036 " and the ground plane disk was 1.7" in diameter. The basic test is depicted in Figure 4-1. The function generator was set to $20 \mathrm{~V}_{\mathrm{p}-\mathrm{p}}$ and an oscilloscope was used to measure the received signal at a variety of frequencies: $10,50,500$ and $1000 \mathrm{kHz}$. For all measurements, the loop was displaced below the receive monopole by $15 / 8$ " as depicted in Figure 4-2; that is, the center of the transmit coil was below the monopole. The received voltage as a function of distance is shown in Figure 4-3.

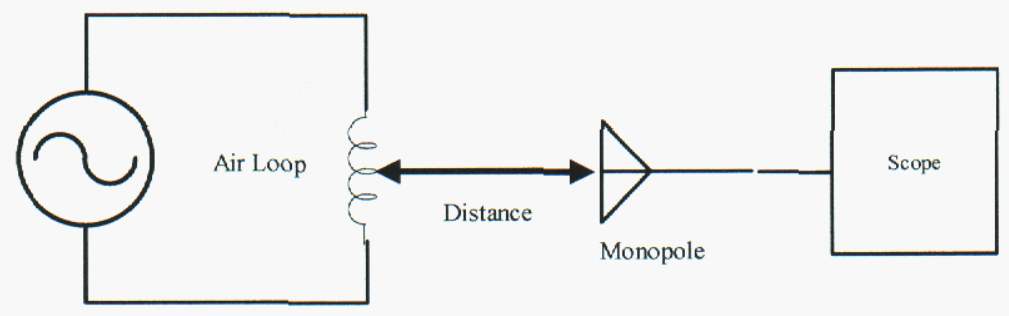

Figure 4-1. Air-loaded Loop and Monopole 


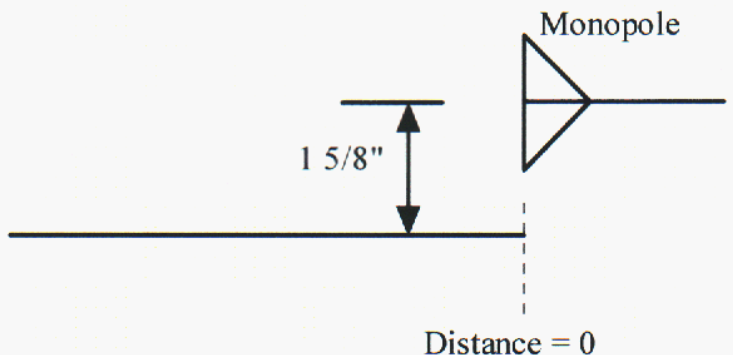

Figure 4-2. Lateral Displacement of Loop from Monopole

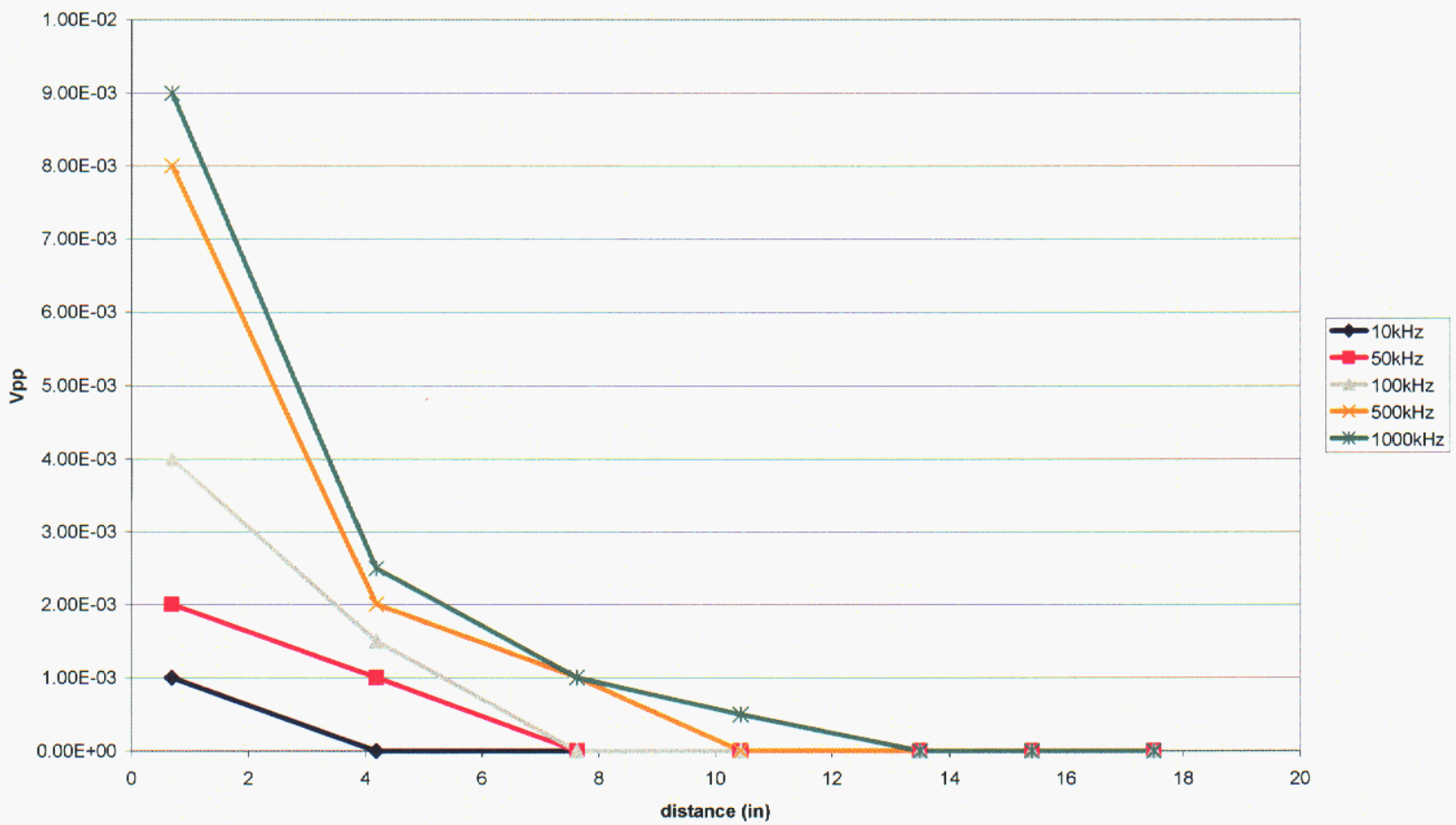

Figure 4-3. Received Voltage vs. Distance (Air-loaded Loop/Monopole)

It is apparent the received voltage has a strong dependence upon frequency. The test was repeated with a ferrite-loaded loop. Again, 120 turns of 30 -gauge wire were wound around a 0.25 " diameter ferrite rod that was 1.7 " long. The permeability of the ferrite rod was $\mu_{\mathrm{r}}=125$. The basic test is depicted in Figure 4-4; the displacement of the center of the ferrite-loaded loop was displaced as shown in Figure 4-2. The results are plotted in Figure 4-5. The curves at $\mathrm{f}=50$ and $100 \mathrm{kHz}$ were identical as were those at 500 and $1000 \mathrm{kHz}$. 


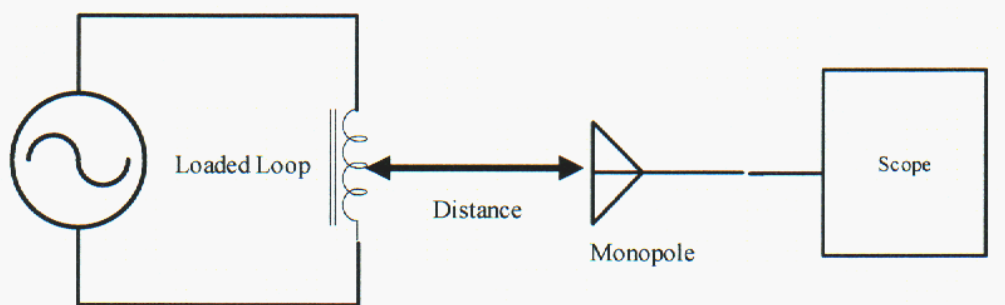

Figure 4-4. Air-loaded Loop and Monopole

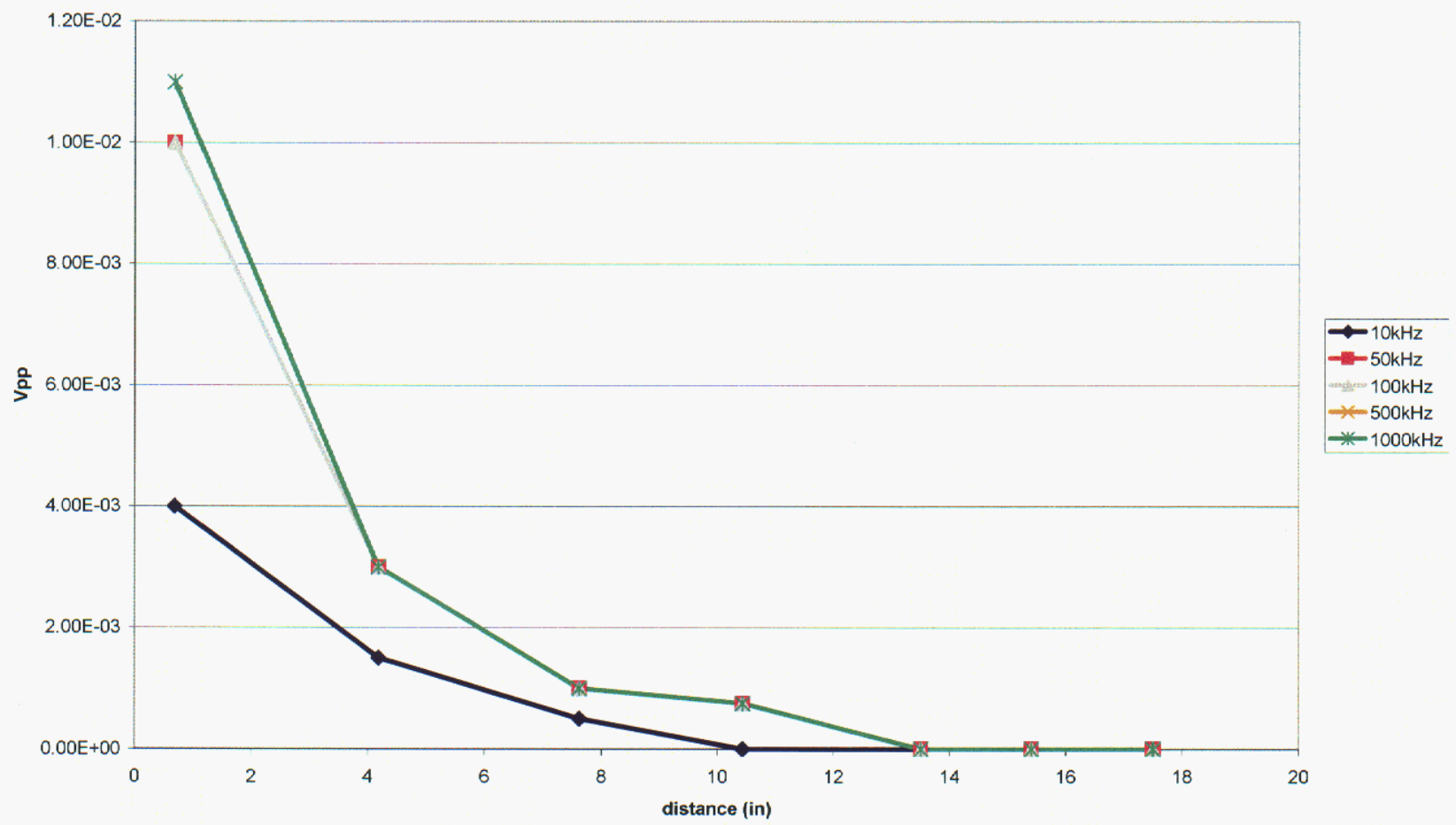

Figure 4-5. Received Voltage vs. Distance (Ferrite-loaded Loop/Monopole)

For the purpose of comparison, the receive voltage was measured with the monopole replaced by a second ferrite-loaded loop that was 0.5 " in diameter and 2 " long. It had correspondingly more turns, about 140 in this case. The basic test is depicted in Figure 4-6 and the results are given in Figure 4-7. It should be noted that the test was run with the loops parallel and in the same plane; there was no displacement such as that depicted in Figure 4-2. 


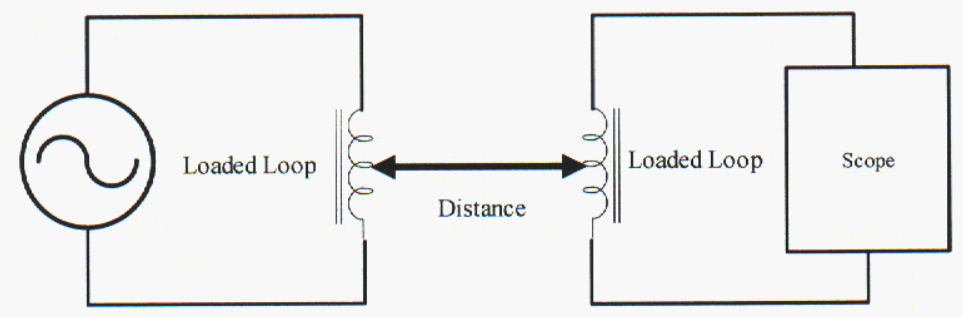

Figure 4-6. Ferrite-loaded Loops Test

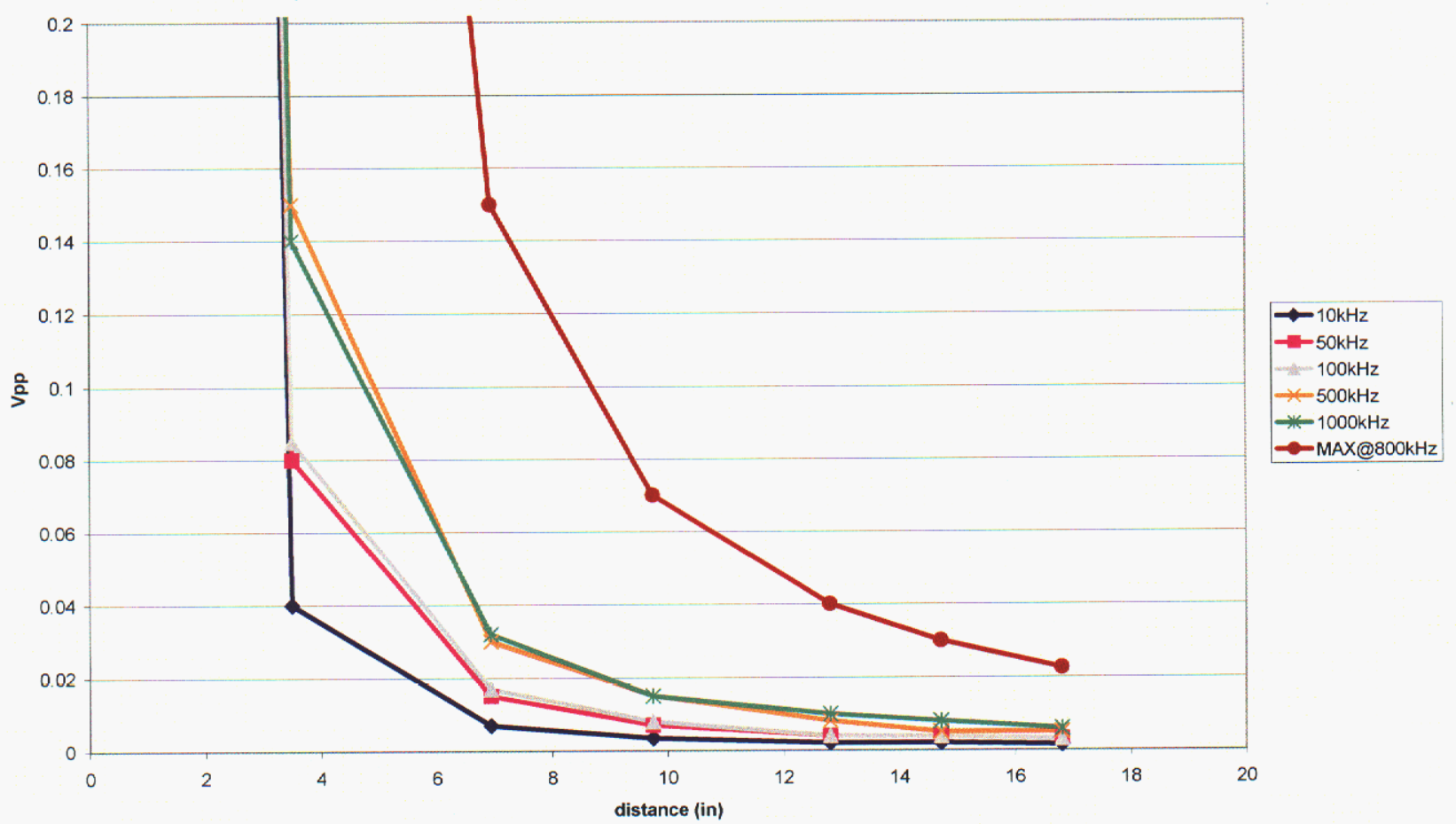

Figure 4-7. Received Voltage vs. Distance (Ferrite-loaded Loop/Ferrite-loaded Loop)

It is apparent that the coupling between the two magnetic antennas is significantly greater than that between a monopole and a coil displace from the axis of the electric antenna. 


\subsection{In-tube Tests}

In-tube tests were performed using the prototype sensor for three conditions: 1) an oversized, metallic tube (2.925" ID, wall thickness $0.150 "), 2$ ) a smaller metallic tube that was a relatively close fit to the outer diameter of the sensor housing without the guide mechanism (2.075"ID, wall thickness 0.050 "), and 3 ) an Inconel tube (3.45"ID, wall thickness $0.100 ")$. This section will document the test configurations, discuss the data processing method and then present the results.

The test configuration utilized the electronics shown in Figure 2-3 with one exception; the function generator was used to produce the sinusoidal signal applied to the fiber optic transmitter rather than the computer. Specifically, the data acquisition/control board and low-pass filter (LPF) were not used in this testing. This was done to facilitate the testing; the computer had difficulty addressing the Ztech controller and acquiring data simultaneously. Thus, the signal was produced by the function generator and the data received by the acquisition board with the computer.

For all tests, the function generator was set to $20 \mathrm{~V}_{\mathrm{p}-\mathrm{p}}$ and the computer acquired the data over a 4 second period, the interval between samples was $\Delta t=10 \mu \mathrm{sec}$. The first two tests were static; that is, the sensor was placed at a specific location (and orientation) within the tube under test and data acquired. The basic tube configuration for the first two tests is shown in Figure 4-8. Tests were performed with the sensor located within: 1) an intact section of tube (the sensor was located within the section of tube for which $\mathrm{z}<0$ ), 2) centered about a semicircular slit (the sensor was centered about the $x-y$ plane) and 3 ) in a half-tube section (the sensor was located in the half-tube section such that there was no metal over the housing). In all three cases consider a coordinate system oriented such that the $\mathrm{z}$ axis was coincident with the axis of the tube, the $\mathrm{y}$ axis is vertical and the $x$ axis orthogonal to the other two. For case 2), the $y$-axis went through the middle of the semicircular slit, which had a longitudinal length of about $2 \mathrm{~cm}$. In case 3 ), the tube above the $x-y$ plane was removed for a total of 4 inches about the sensor housing. The above tests were run for two orientations of the sensor transmit loop: A) with the axis of the coil (multiturn loop) vertical (axis aligned along $y$ ) and B) with the axis of the coil (multi-turn loop) horizontal (axis aligned along x). A photograph of the 2.075" ID tube is shown in Figure 4-9.

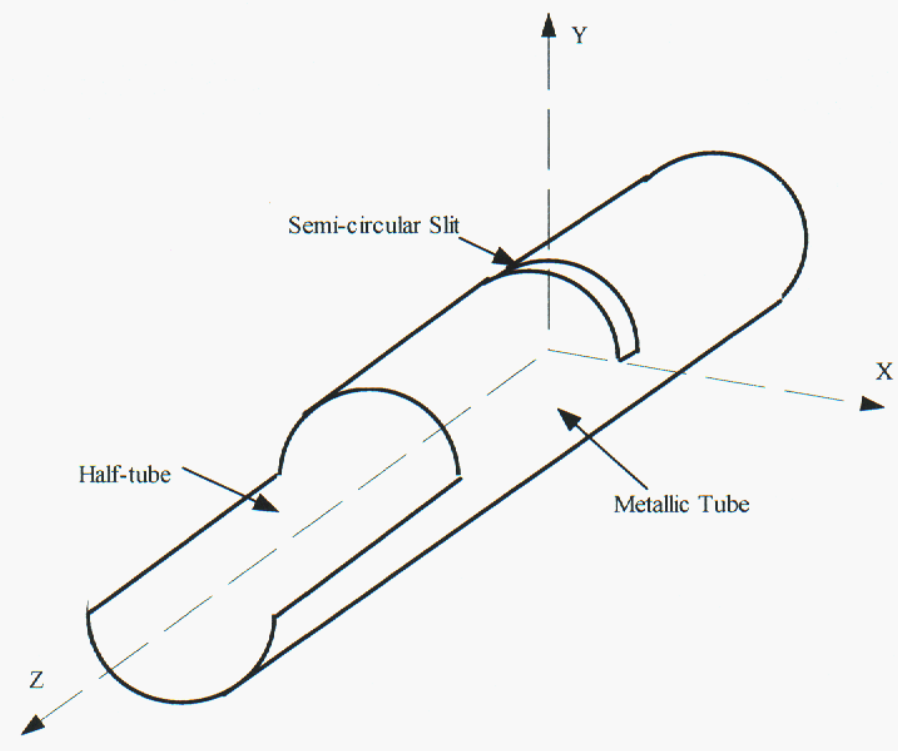


Figure 4-8. Tube Configuration for Static Tests

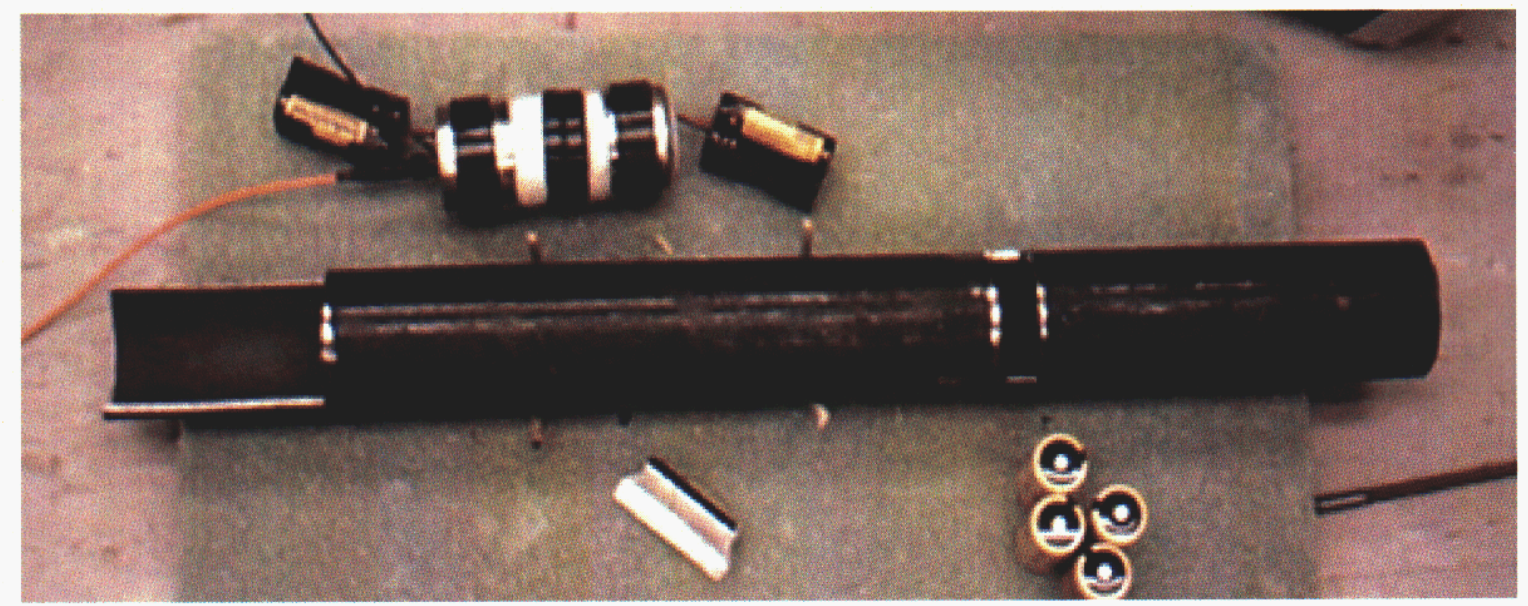

Figure 4-9. Photograph of Tube Testing Configuration

Regardless of the orientation of the sensor, or its location within the tube, the computer data acquisition board was used to digitize the voltage signal of interest, $V_{\text {raw }}$. Once the data file was created, the processing was completed in the following steps:

1) Perform a Fourier transform to obtain the data in the frequency domain.

2) Perform a band-pass filter operation on the frequency domain data.

3) Recreate the discretized frequency spectrum.

4) Transform back to the time domain.

For convenience, a built-in MathCad function was used in Step 1) to perform the Fourier transform. This built-in function was limited to a data sequence of 262,144 points. Thus, the raw data was truncated to the first 262,144 points prior to the Fourier transform operation. The transformed data was placed in vector $\widetilde{V}$ for further processing. The Fourier transform operates on a sequence of length $\mathrm{M}$ and produces a vector of the same length in the frequency domain.

We have

$$
\widetilde{V}_{n}=\mathfrak{S}\left(V_{r a w}\right)=\frac{1}{\sqrt{M}} \sum_{m} V_{r a w_{m}} e^{j \frac{2 \pi n}{M} m}
$$

The index $m$ varies over the range $m=1,2, \ldots M$ with $M=2^{18}$. The resultant discrete frequency domain is a vector ranging over $n=1,2, \ldots N$ with $N=2^{18}$. Since the input sequence, $V_{\text {raw }}$, is real valued, the magnitude of the frequency spectrum is symmetric about its midpoint while the phase response is skew symmetric about this same point. The time interval between sampled voltage data points was $10 \mu \mathrm{sec}$. Thus,

$$
\Delta t=10 \times 10^{-6} \quad \mathrm{sec}
$$


The frequency of the $n^{\text {th }}$ component in the discrete output frequency spectrum is then determined as

$$
f_{n}=\frac{n}{M \Delta t}
$$

Thus, for a particular source frequency setting we can filter the spectrum and emphasize the response at that specific frequency of interest. In the presentation of the data that follows, the filter was set according to the output of the frequency generator at that point in time; that is, since the function generator output frequency varied over time and there was a delay between each test, we simply filter the raw data knowing the frequency at that point in time, which is obvious upon inspection of the spectrum, rather than a fixed value such as $10 \mathrm{kHz}$. For example, the nominal frequency for the tube tests was $f=10 \mathrm{kHz}$, as set by the dial on the front panel of the instrument. However, for any given test the frequency was not, in fact, exactly equal to $10 \mathrm{kHz}$. Typically, the band-pass filter was set to weight the desired component and the surrounding ten lines at unity while weighting all other components in the transformed spectrum to zero. Once this was accomplished, the magnitude spectrum was made symmetric about the midpoint while the phase spectrum was forced to be skew-symmetric. To complete the processing, the inverse transform was then computed as

$$
V_{\text {proc } n}=\mathfrak{I}^{-1}(\widetilde{V})=\frac{1}{\sqrt{M}} \sum_{m} \widetilde{V}_{m} e^{-j \frac{2 \pi n}{M} m}
$$

We then plot the magnitude of $V_{\text {proc }}$ versus time, that is, $\left|V_{\text {proc }}(t)\right|$ and compare the values obtained from the sensor located near a defect to that when the sensor is in an intact portion of the tube (no defect). In the actual ITR application, $\left|V_{\text {proc }}(t)\right|$ would be observed over time and the location of defects identified.

We begin by considering for first set of tests, those performed with the 2.925 " ID diameter metal tube. As stated above, data was acquired over a 4 second span at an interval of $10 \mu \mathrm{sec}$. This produced 400,000 data points. Since the number of data points was truncated to 262,144 , the plots shown below only have data out to 2.62 seconds. Figure $4-10$ shows the response when the sensor was located in cylindrical section of the test tube; that is, a section without defects. For this particular plot, the axis of the coil (multi-turn loop) was vertical, or parallel to the y-axis. It should be noted, that rotation of the sensor in a cylindrical portion of the tube produced no change in the response. 


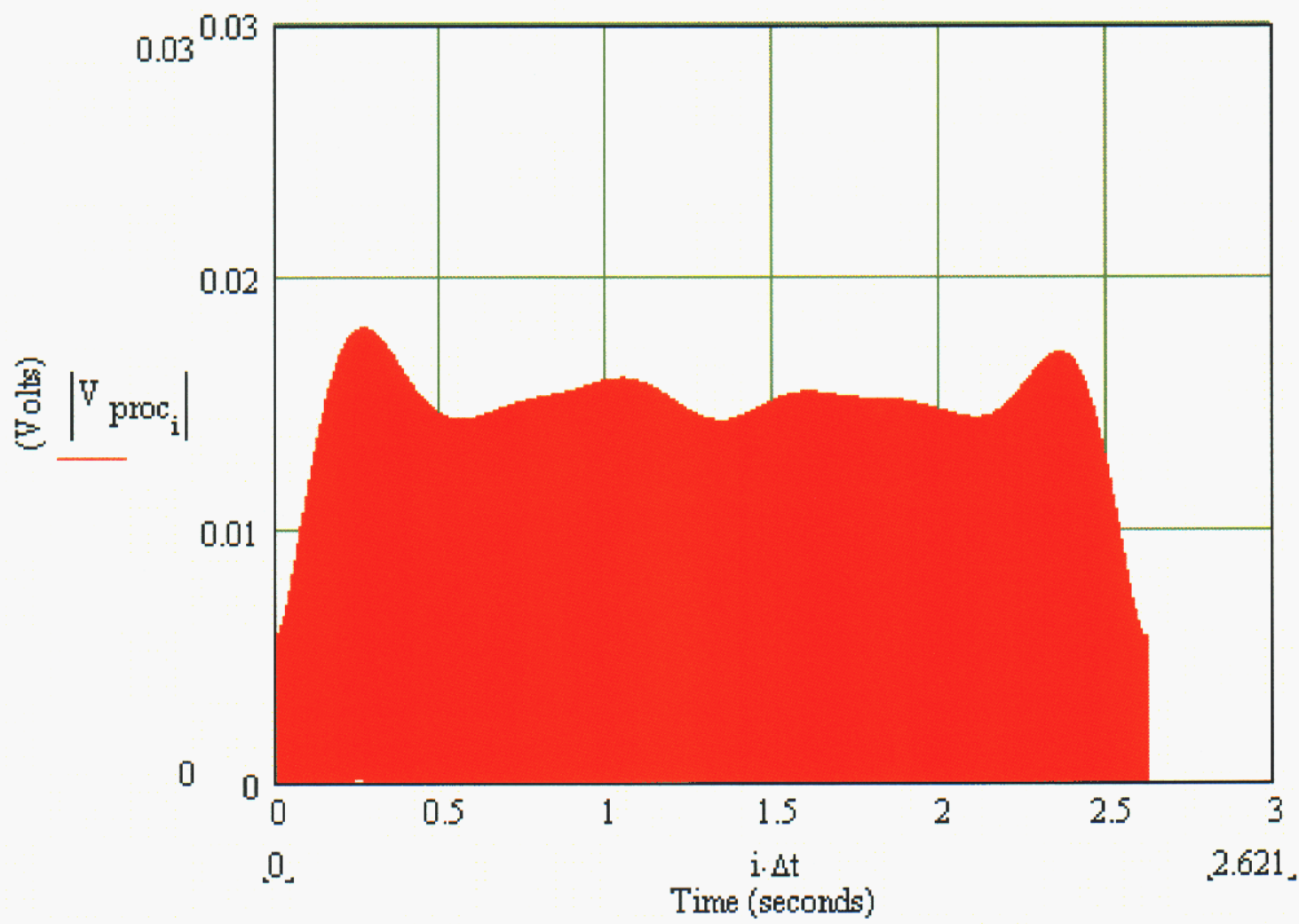

Figure 4-10. Test Case 1), No Defect

The sensor was then moved to the location of the semicircular slit depicted in Figure 4-8. With the sensor in any orientation, that is, with the axis of the transmit antenna horizontal or vertical, the nominal signal level did not change from that shown in Figure 4-10. Thus, the prototype ITR sensor did not have the ability to sense the semicircular defect.

The sensor was then moved to the half tube section shown on the left-hand end of Figure 4-8. First the sensor was positioned so that the axis of the transmit antenna (the coil) was horizontal, the resulting output voltage after filtering is shown in Figure 4-11. The nominal voltage is close to the same as that shown in Figure 4-10. It should be noted that having the axis of the sensor horizontal was the consistent with the proposed operation of the ITR unit; that is, it was supposed to detect defects broadside to the loop antenna. The sensor was then rotated 90 degrees so that the axis of the transmit coil was vertical. There are portions of the curve that show a larger response but the nominal voltage of the 2.62 second interval is not much different from the no defect case. 


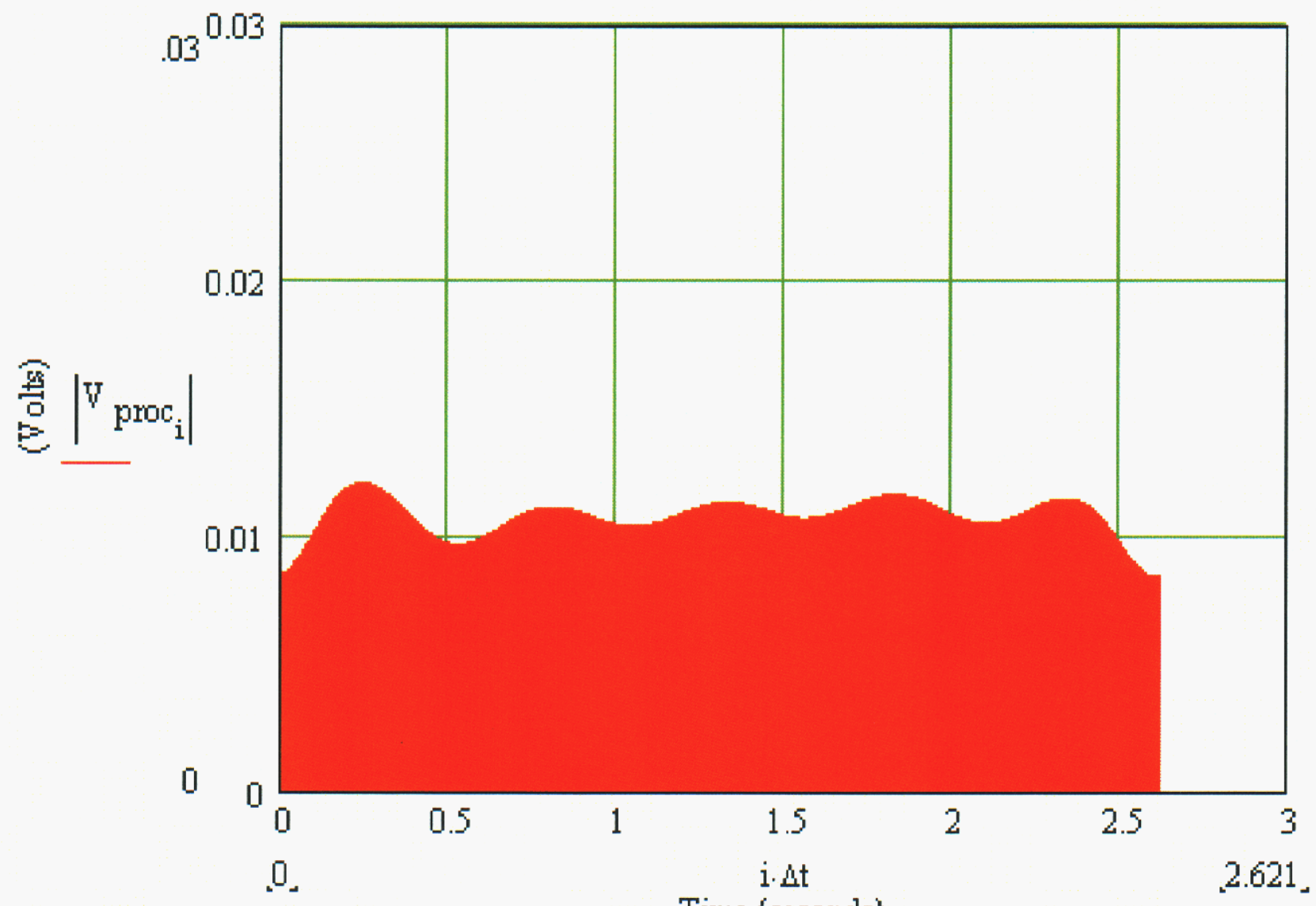

Time (seconds)

Figure 4-11. Test Case 1), Half-tube Defect (Coil Horizontal) 


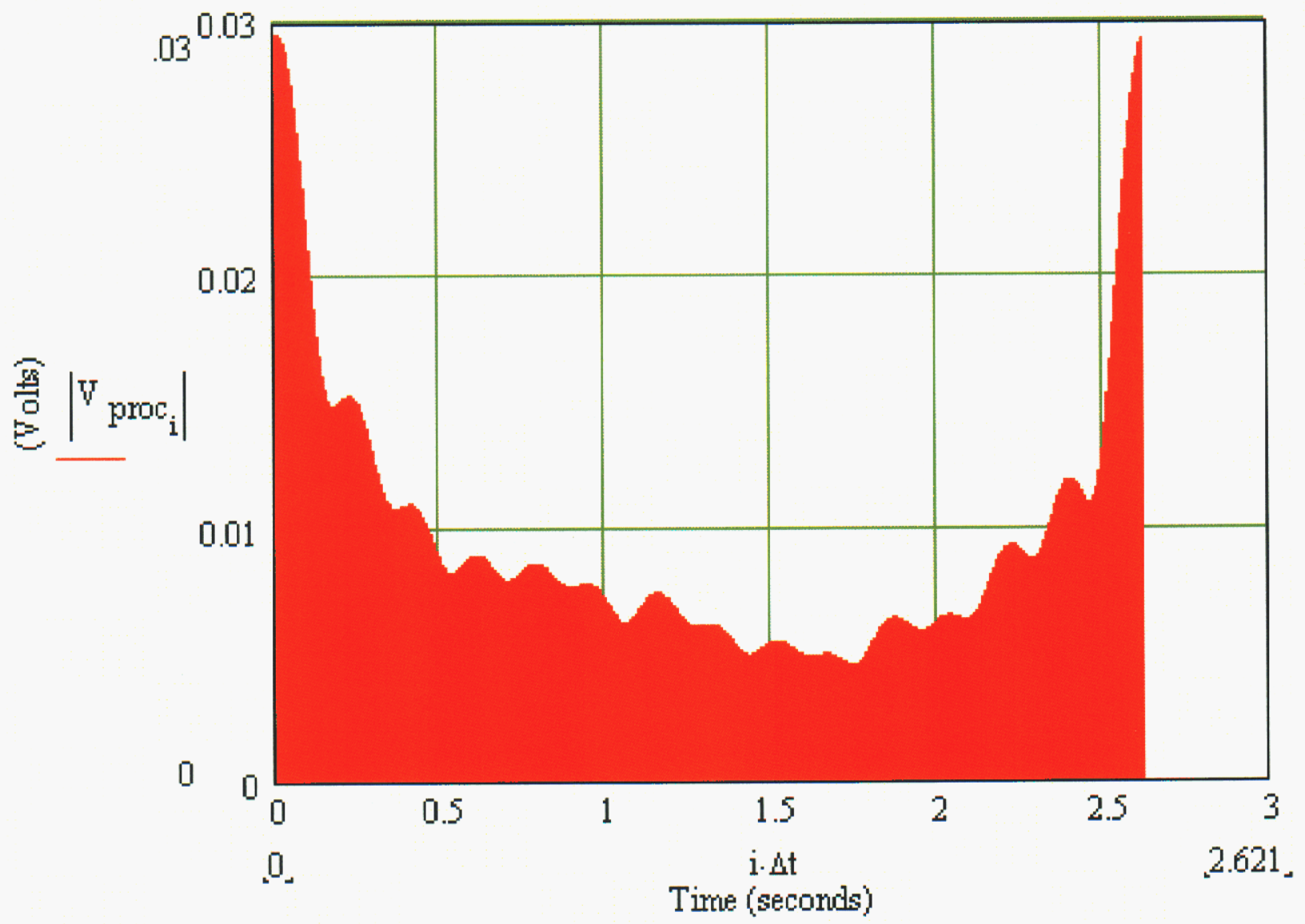

Figure 4-12. Test Case 1), Half-tube Defect (Coil Vertical)

A second tube that was approximately the same outside diameter as the sensor was fabricated and the sequence was repeated, this is Test Case 2) referenced above. With the sensor in an intact portion of the tube, the measured voltage, after filtering, is given in Figure 4-13. Figure 4-14 shows the measured response with the sensor oriented with the axis of the coil horizontal. The received voltage in this case is only slightly greater; the nominal value is essentially the same. When the sensor was rotated 90 degrees, in the same tube, the measured voltage increased by more than an order of magnitude. This is shown in Figure 4-15. 


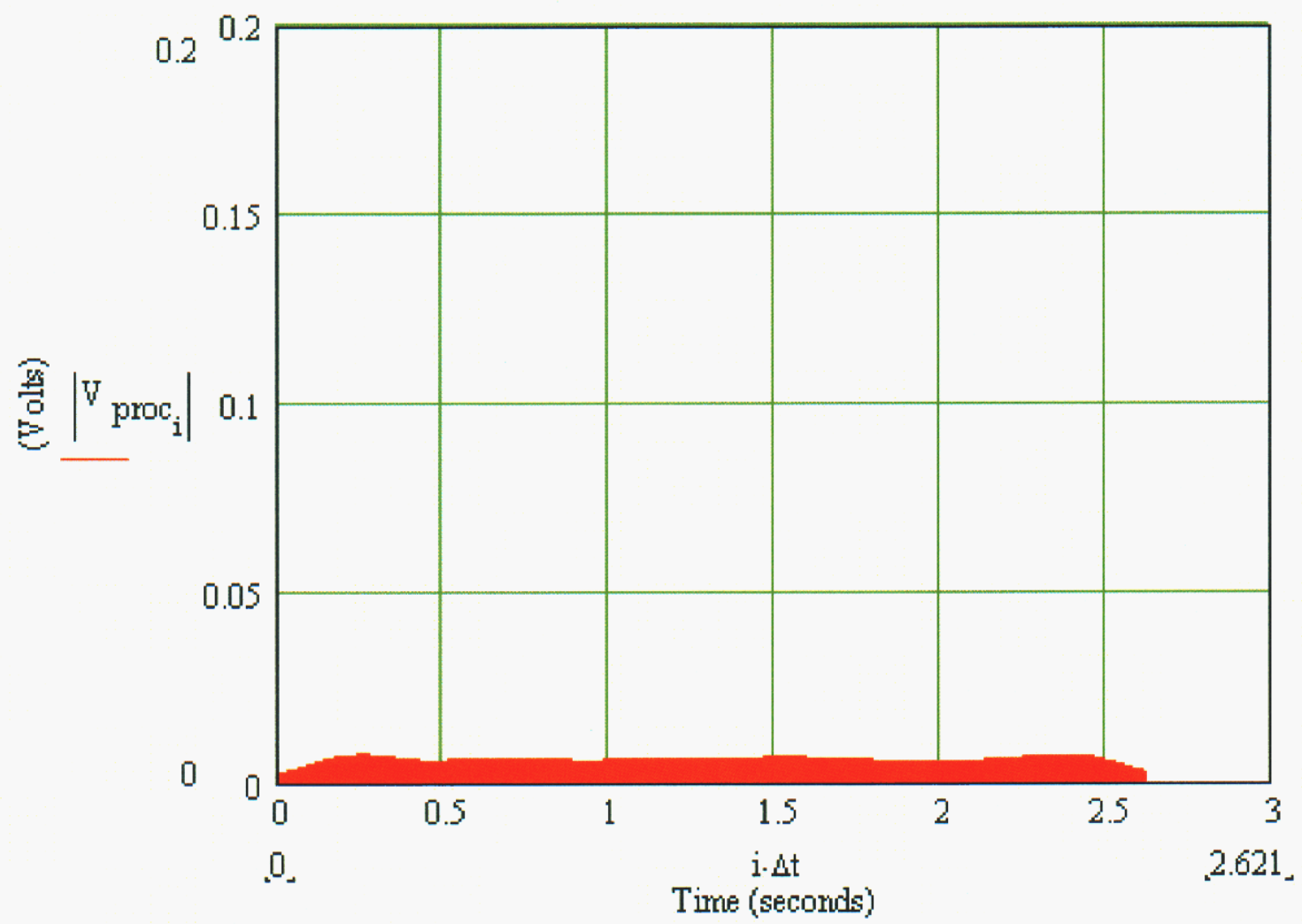

Figure 4-13. Test Case 2), No Defect

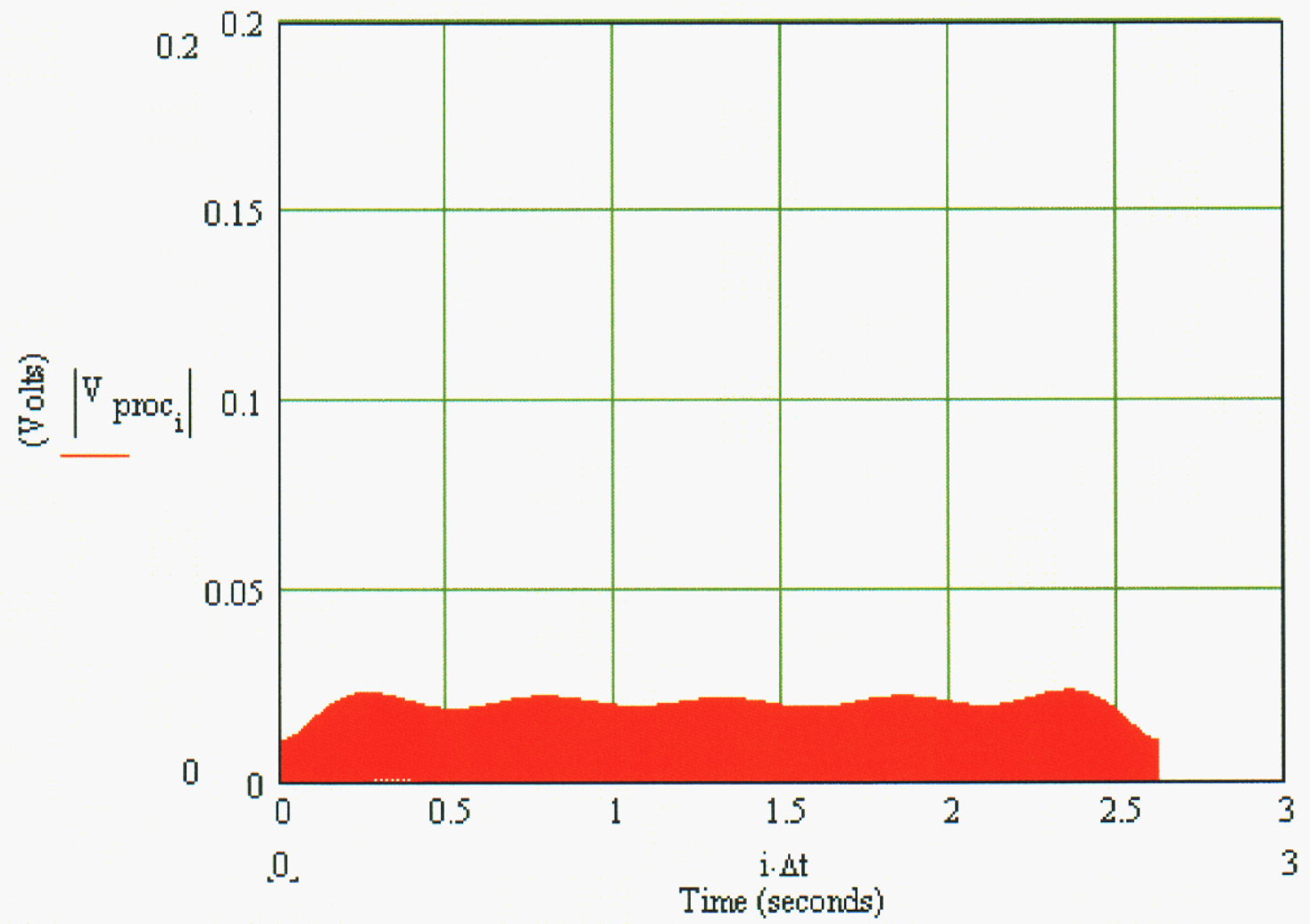

Figure 4-14. Test Case 2), Half-tube Defect (Coil Horizontal) 


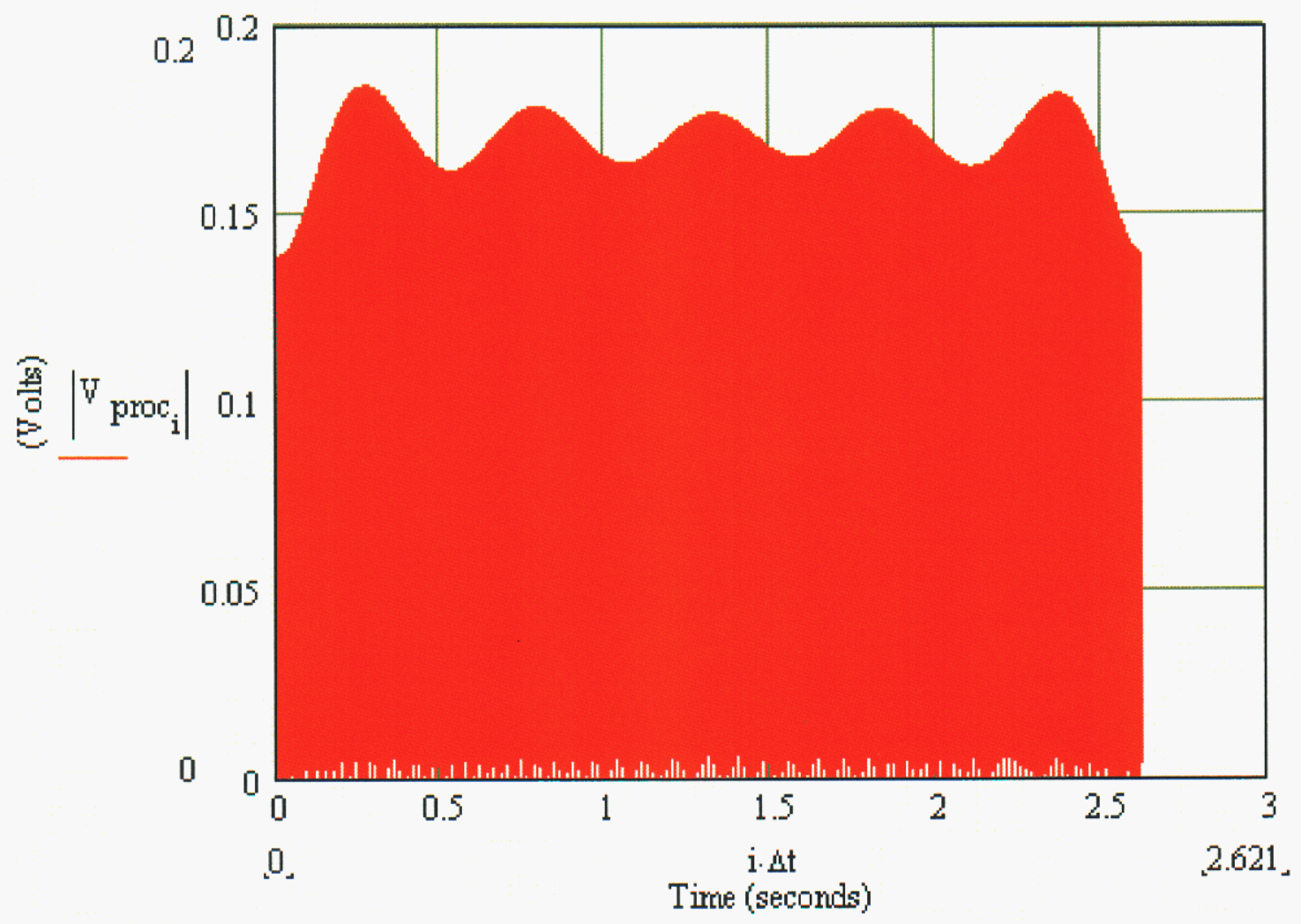

Figure 4-15. Test Case 2), Half-tube Defect (Coil Vertical)

The third test case was run using the computer to generate and acquire the signal; however, when the prototype ITR sensor was centered inside the 3.45" diameter Inconel tube (with no defects), no observable difference in the signal was noted. 


\section{MECHANICAL DESIGN}

The Mechanical Design of the Probe is described in reference number 6, which is an MS thesis by Mr. Sean Brooks, one of the graduate students who was supported by the ITR project.

Copies are available from NMSU library or can be provided by contacting the project principal investigators. 
This page intentionally left blank 


\section{Electromagnetic Modeling and Simulation}

The proposed electromagnetic modeling for this effort was to be limited to use of CTUBE, provided by Sandia National Laboratories. It was envisioned that this code would be used to determine the impact of off-axis sensor location and misalignments. However, the code was not fully functional for the off-axis sensor case and its formulation did not afford the possibility of including the presence of defects. Thus, several other approaches were considered during the course of this investigation. This included the development of a finite element code, FEMTUBE, to model the cylindrical tube geometry incorporating defects as changes in the material properties of some of the elements. This turned out to be more of an effort than originally thought and other techniques were applied to the problem. These included a finite element analysis using a commercially available code. In conjunction with NMSU's Physical Science Laboratory, Ansoft's High Frequency Structure Simulator (HFSS) was also used to model the steam generator tube without, and with, defect. Finally, the approach was entirely shifted by using an integral equation code, the Numerical Electromagnetics Code (NEC). These are discussed in the following sections.

\subsection{CTUBE}

CTUBE was a computer code developed by Sandia National Laboratories to model the fields in and around a cylindrical geometry such as those of interest in the borehole radar application. The use of this code in the steam generator tube application was discussed in Sandia report SAND970170 as well as the ITR proposal. CTUBE was based upon the work of Wait and Hill (James R. Wait and David A. Hill, "Electromagnetic Fields of a Dipole Source in a Circular Tunnel Containing a Surface Wave Line," International Journal of Electronics, 1977, Vol. 42, No. 4, pp 377-391.). This work was primarily done to model cylindrical exclusions (such as a mine) in a larger dielectric medium. That is, a cylindrical dielectric medium within a second infinite dielectric. The borehole radar application is somewhat different than the configuration necessary to inspect steam generator tubes. The ITR problem consists of the interior cylindrical source region, surrounded by a metallic wall of finite thickness, which is further enclosed by an exterior region filled with various objects and materials. Also, the frequency of operation in the borehole radar case was substantially higher than that required for the fields to penetrate the wall of the steam generator tube. Thus, the radiation efficiency of the transmit and receive antennas in that application, in addition to the absence of a metallic enclosure, allowed the hardware to function as a radar and be modeled based upon the work of Wait and Hill.

The CTUBE code was used by Sandia to make preliminary calculations that indicated the ITR method had several advantages above the eddy current method presently used to inspect steam generator tubes. First, the proposed ITR technique did not require comparison to a reference sensor located in a "standard" tube. Second, the proposed ITR method would be capable of identifying the circumferential location of a defect without rotation of the sensor itself. Third, it was conjectured that the inspection rate using the ITR technique would be considerably faster than the eddy current methods presently used.

The primary purpose of using CTUBE in this investigation was to determine the sensitivity of the ITR method to misalignment of the sensor; that is, it was envisioned that the anticipated increase in axial electric field could be quantified as a function of the radial displacement of the sensor from the axis of the tube. However, the code originally supplied by Sandia was not fully 
functional for the off-axis case. Thus, it was decided to pursue other modeling techniques that would incorporate a finite thickness tube wall embedded between two media (the interior and exterior regions).

\subsection{FEMTUBE}

The problem geometry is depicted in Figure 6-1. The computational domain consists of: 1) the interior region, the air-filled region inside the tube, 2) the tube wall itself, and 3 ) the exterior region. Figure 6-1 shows a small defect in the exterior wall of the tube. The overall problem domain was truncated by using a perfectly matched layer at the outer boundary of the exterior region. To model defects, the material properties of specific finite elements within, or on, the tube wall were changed; that is, instead of the finite element having the properties of Inconel it could be changed to air, or, for an intermediate defect some appropriate electrical parameter in between. The finite-element method (FEM) was used to solve Maxwell's equations, subject to the appropriate boundary conditions, to determine the axial electric field for excitation with a magnetic source (multi-turn loop antenna).

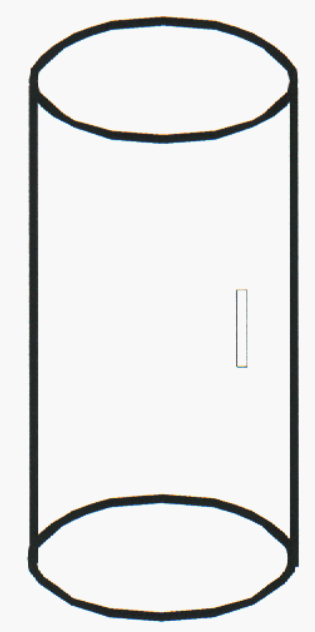

Figure 6-1. Steam Generator Tube with Longitudinal External Defect

We begin with Maxwell's equations in time-harmonic form:

$$
\begin{gathered}
\bar{\nabla} \times \bar{E}=-j \omega \mu_{o} \overline{\overline{\mu_{r}}} \bar{H}-\overline{M_{i}} \\
\bar{\nabla} \times \bar{H}=-j \omega \varepsilon_{o} \overline{\overline{\varepsilon_{r}}} \bar{E}+\overline{J_{i}}
\end{gathered}
$$

This representation allows for magnetic as well as electric losses given the following tensor forms. 


$$
\begin{gathered}
\overline{\overline{\varepsilon_{r}}}=\left[\begin{array}{ccc}
\varepsilon_{r x}-j \frac{\sigma_{x}}{\omega \varepsilon_{o}} & 0 & 0 \\
0 & \varepsilon_{r y}-j \frac{\sigma_{y}}{\omega \varepsilon_{o}} & 0 \\
0 & 0 & \varepsilon_{r z}-j \frac{\sigma_{z}}{\omega \varepsilon_{o}}
\end{array}\right] \\
\overline{\overline{\mu_{r}}}=\left[\begin{array}{ccc}
\mu_{r x}-j \frac{\sigma_{x}^{*}}{\omega \mu_{o}} & 0 & 0 \\
0 & \mu_{r y}-j \frac{\sigma_{y}^{*}}{\omega \mu_{o}} & 0 \\
0 & 0 & \mu_{r z}-j \frac{\sigma_{z}^{*}}{\omega \mu_{o}}
\end{array}\right]
\end{gathered}
$$

In addition, these tensor forms allow the perfectly matched layer of Berenger to be implemented in a convenient manner.

For this problem the impressed electric current density, $\bar{J}_{i}$, will be zero. Since we will use a magnetic antenna, $\bar{M}_{i} \neq 0$, and the electric field form will be utilized. To achieve this form we solve for $\bar{H}$ in the first curl equation and upon substitution into the second we have the vector wave equation.

$$
\bar{\nabla} \times \bar{\mu}_{r}^{-1} \bar{\nabla} \times \bar{E}-\omega^{2} \mu_{o} \varepsilon_{o} \bar{\varepsilon}_{r} \bar{E}=\bar{\nabla} \times \bar{\mu}_{r}^{-1} \overline{M_{i}}
$$

Apply the method of weighted residuals by forming an appropriate inner product.

$$
\iiint_{V} \bar{T} \cdot\left\{\bar{\nabla} \times \bar{\mu}_{r}^{-1} \bar{\nabla} \times \bar{E}-\omega^{2} \mu_{o} \varepsilon_{o}=\overline{\varepsilon_{r}} \bar{E}\right\} d v=-\iiint_{V} \bar{T} \cdot \bar{\mu}_{r}^{-1} \bar{\nabla} \times \overline{M_{i}} d v
$$

By a vector identity and Gauss' divergence theorem the first term is split into volume and surface integral terms.

$$
\iiint_{V}(\bar{\nabla} \times \bar{T}) \cdot\left(\bar{\mu}_{r}^{-1} \bar{\nabla} \times \bar{E}\right) d v-\oiint_{S} \bar{T} \times\left(\bar{\mu}_{r}^{-1} \bar{\nabla} \times \bar{E}\right) \cdot \overline{d s}-\omega^{2} \mu_{o} \varepsilon_{o} \iiint \bar{T} \cdot \bar{\varepsilon}_{r} \bar{E} d v=-\iiint_{V} \bar{T} \cdot \bar{\mu}_{r}^{-1} \bar{\nabla} \times \overline{M_{i}} d v
$$

The domain is discretized and a continuous approximation to the electric field, due to excitation by the magnetic source, is determined throughout the domain simply by specifying the material properties throughout the region. The computational size of the domain is restricted by imposing 
a perfectly matched layer (PML) at the perimeter. As shown in Figure 6-2, the free space region outside the heat exchanger tube is also modeled using the finite element method. At the outer edge of the top, bottom and cylindrical surfaces PML elements are placed adjacent to the perimeter. We select the permeability and permittivity tensors (for a boundary parallel to the top and bottom surfaces of the domain) as shown below.

$$
==\left[\begin{array}{ccc}
1-j \frac{\sigma^{*}}{\omega \mu_{o}} & 0 & 0 \\
0 & 1-j \frac{\sigma^{*}}{\omega \mu_{o}} & 0 \\
0 & 0 & \left(1-j \frac{\sigma^{*}}{\omega \mu_{o}}\right)^{-1}
\end{array}\right]
$$

The permeability, permittivity and conductivities ( $\Phi$ and $\left.\Phi^{*}\right)$ are selected to minimize the reflection coefficient at all angles and also produce dissipation so that the minimal energy is present by the time the wave propagates to the edge of the computational domain. The permeability and permittivity tensors are similarly selected on the outer cylindrical surface as well. 


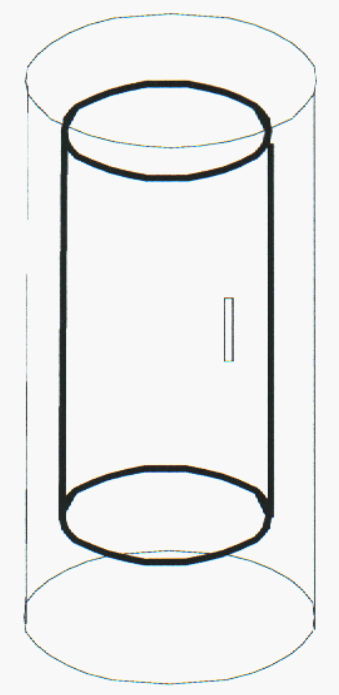

\section{Figure 6-2. Computational Domain showing Exterior Region}

FEMTUBE was implemented using pentahedral and hexahedral elements. That is, the region along the centerline of the tube was discretized using five-sided elements while the remainder of the domain was split into 6-sided shapes.

The code was run on a geometry corresponding to that of the prototype sensor within the 3.5 " inside diameter Inconel tube. The transmit antenna was modeled as an infinitesimal magnetic radiator located along the axis of the tube. The on-axis electric field was then calculated without, and with, a defect present. In this case, the defect was a semicircular slit completely cut through the wall of the tube; the longitudinal extent of the slit was $2 \mathrm{~cm}$. Figure 6-3 shows the calculated electric field across a cut plane through the domain. The horizontal axis has units of meters, the inside of the 3.5 " ID tube is obvious at $\pm 0.04445 \mathrm{~m}$ to the left and right of center, which is 0 on the horizontal scale. The vertical axis is not scaled to any particular absolute value it should simply be used to quantify the relative difference between the no defect/defect cases. The electric field was calculated along a line in the $y-z$ plane $2 \mathrm{~cm}$ above the point source magnetic transmitter. The solid line is the calculated response with no defect present. The dashed curve is the on-axis field with a semi-circular section removed from the tube. Unfortunately, the results were quite sensitive to the mesh and convergence tests were not possible due to limitations in the matrix solution on the computer for which the code was developed. This approach was abandoned in favor of use of the commercial Ansoft product. 


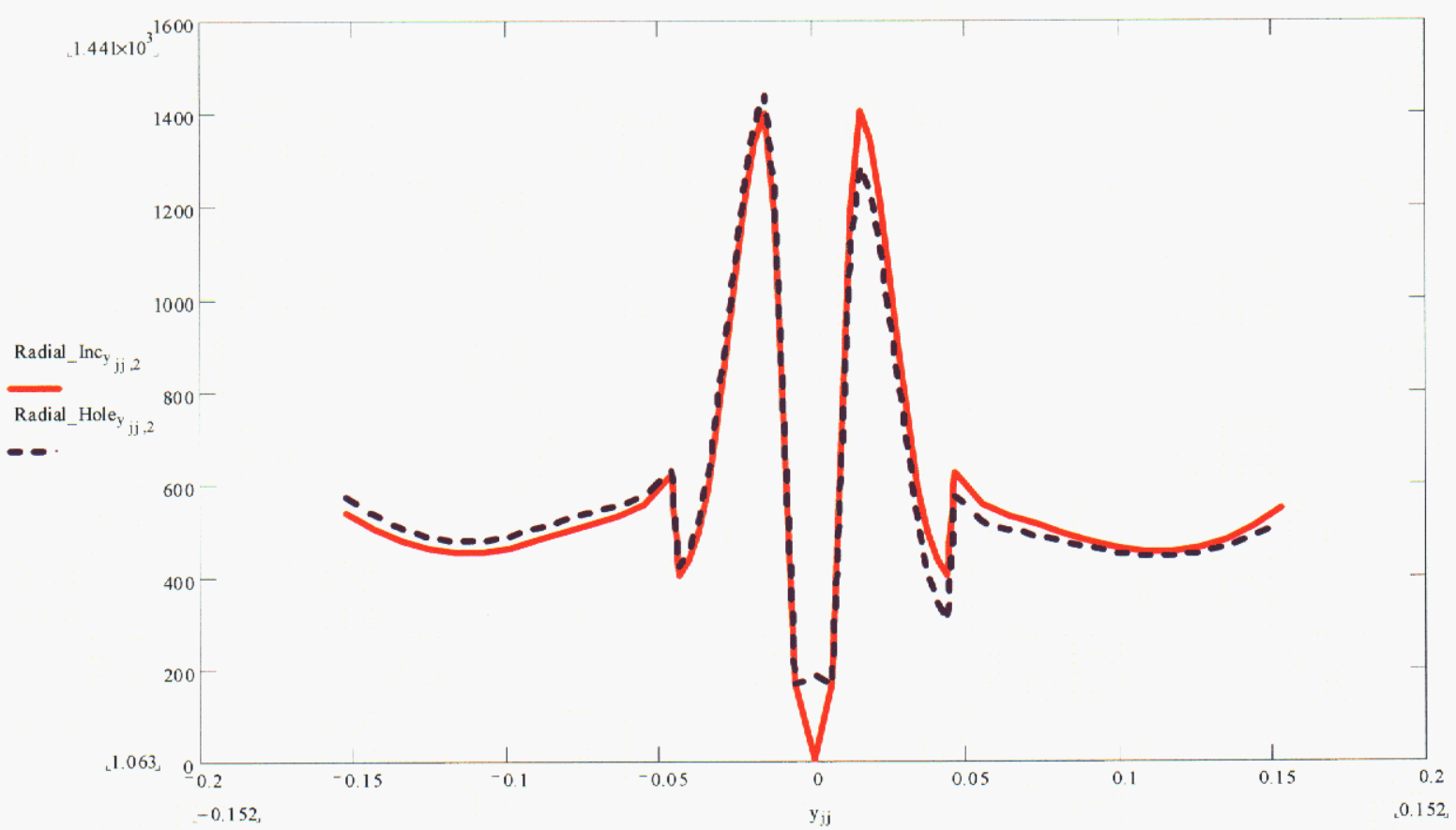

Figure 6-3. Electric Field across the Y-Z Plane $2 \mathrm{~cm}$ above Source

\subsection{High Frequency Structure Simulator (HFSS)}

In conjunction with the Physical Science Laboratory at NMSU, Ansoft's HFSS simulation software was used to model the steam generator tube geometry. Although a widely used code, HFSS does not have the capability to model a point magnetic source; it was necessary to excite the structure by modeling an actual loop antenna located on the axis of the tube. The magnetic source antenna, along with the tube and defect are depicted in Figure 6-4. 


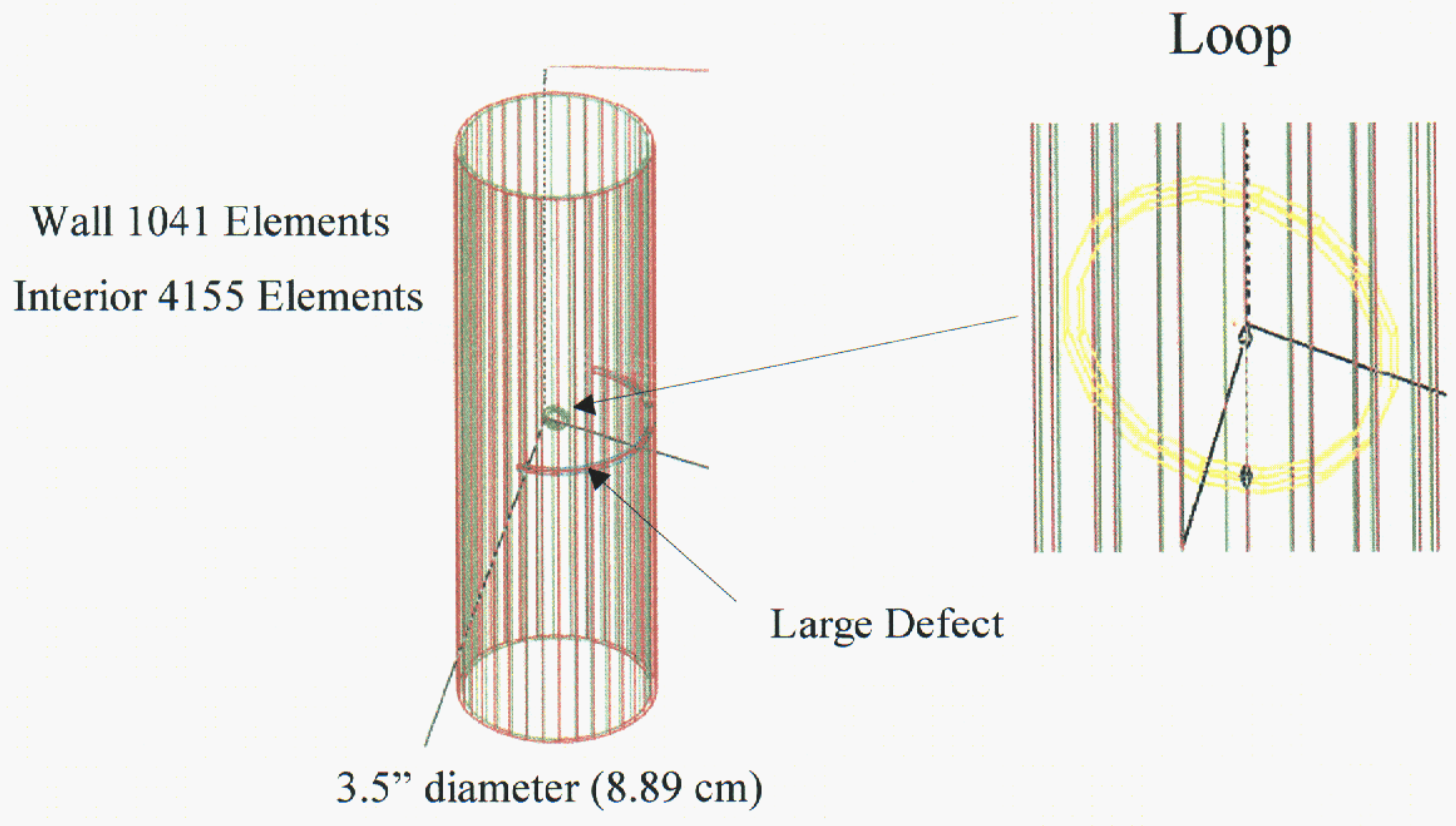

Figure 6-4. HFSS Model

The monopole receive antenna was not modeled. The HFSS code calculates the fields $(\mathrm{E}$ and $\mathrm{H})$ at any point within the domain so it was not necessary to include the details of the monopole receiver. Again, an analysis was done for the two cases, no defect and semicircular defect. The longitudinal extent of the slit was $2 \mathrm{~cm}$. The resulting electric field, parallel to the axis of the tube, is shown in Figure 6-5. The green curve is for no defect; the red curve is for the semicircular slit. It should be noted that the vertical scale is in $\mathrm{dB}$ for Figure 6-5.

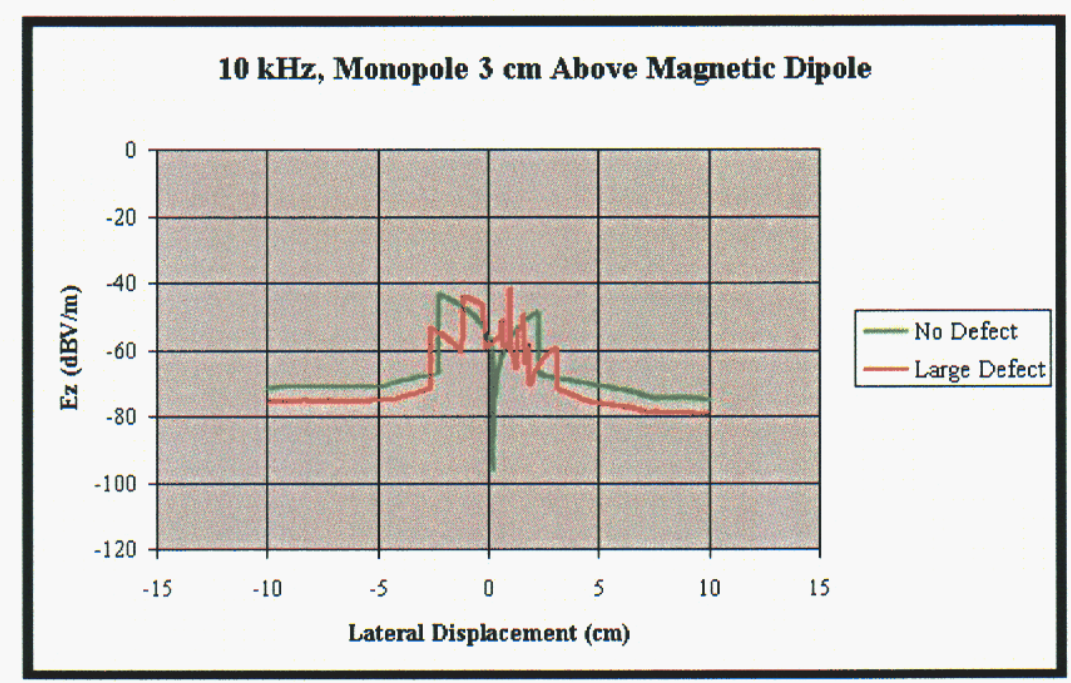

Figure 6-5. Electric Field versus Lateral Displacement 
The modeling performed using HFSS suffered from much the same problem as that encountered with the in-house developed FEMTUBE, the solution of the resulting partial differential equations could not be effectively accomplished at such low frequencies. Basically, the structure was too small with respect to a wavelength (and too much geometric detail was required to implement the magnetic source, or loop) that HFSS failed to provide a solution as the mesh was refined and convergence attempted. Thus, an alternative simulation method was considered to circumvent this problem.

\subsection{Numerical Electromagnetic Code (NEC)}

The numerical electromagnetic code, or NEC, is an integral-equation-based solution of Maxwell's equations. Instead of discretizing an entire domain, it is only necessary to represent the conductors in the system and use Green's functions to compute the fields while enforcing the boundary conditions. This is done using the method of weighted residuals; in electromagnetics the application of said method using integral equations is termed the method of moments (MOM). The NEC MOM code was applied to the geometry shown Figure 6-6. The tube was constructed from 8 -sided polygons, 33 such structures were stacked vertically along the z-axis. The tube was then formed by interconnecting the nodes of the polygons using vertical wires. Close observation of Figure 6-6 shows the transmit loop antenna (positioned along the centerline of the tube) as well as a receive dipole antenna. In this simulation, we simply compare the magnitude of the currents induced in the receive antenna for the no defect and defect cases. The ratio of these two currents would be proportional to the ratio of the axial electric fields for the two cases (and hence the voltage ratio at the receiver). A tube with a defect is shown in Figure 6-7. The resulting current distribution is shown in Figure 6-8 for no defect, in Figure 6-9 for a large defect, which was formed by removing the wires in a semicircle about half the circumference of the tube as well as two and a half 8 -sided polygons. The magnitude of the source was set to the same value in both simulations. Perusal of Figure 6-7 and 6-8 show that no discernable difference in the receive antenna currents is observable in the presence of a defect. Thus, a receiver connected to the terminals of either antenna would read the same voltage. 


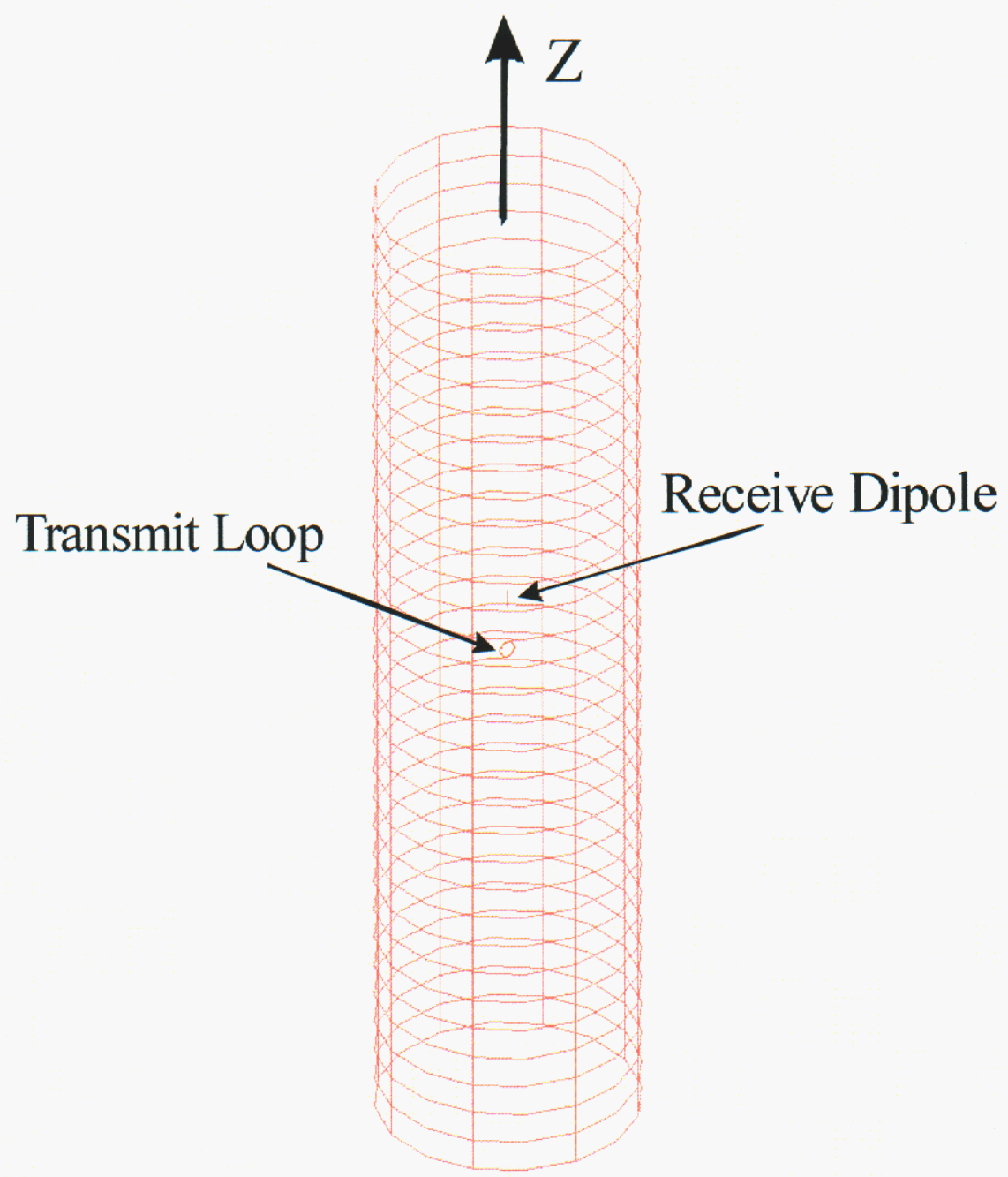

Figure 6-6. View of Wireframe Tube Model 


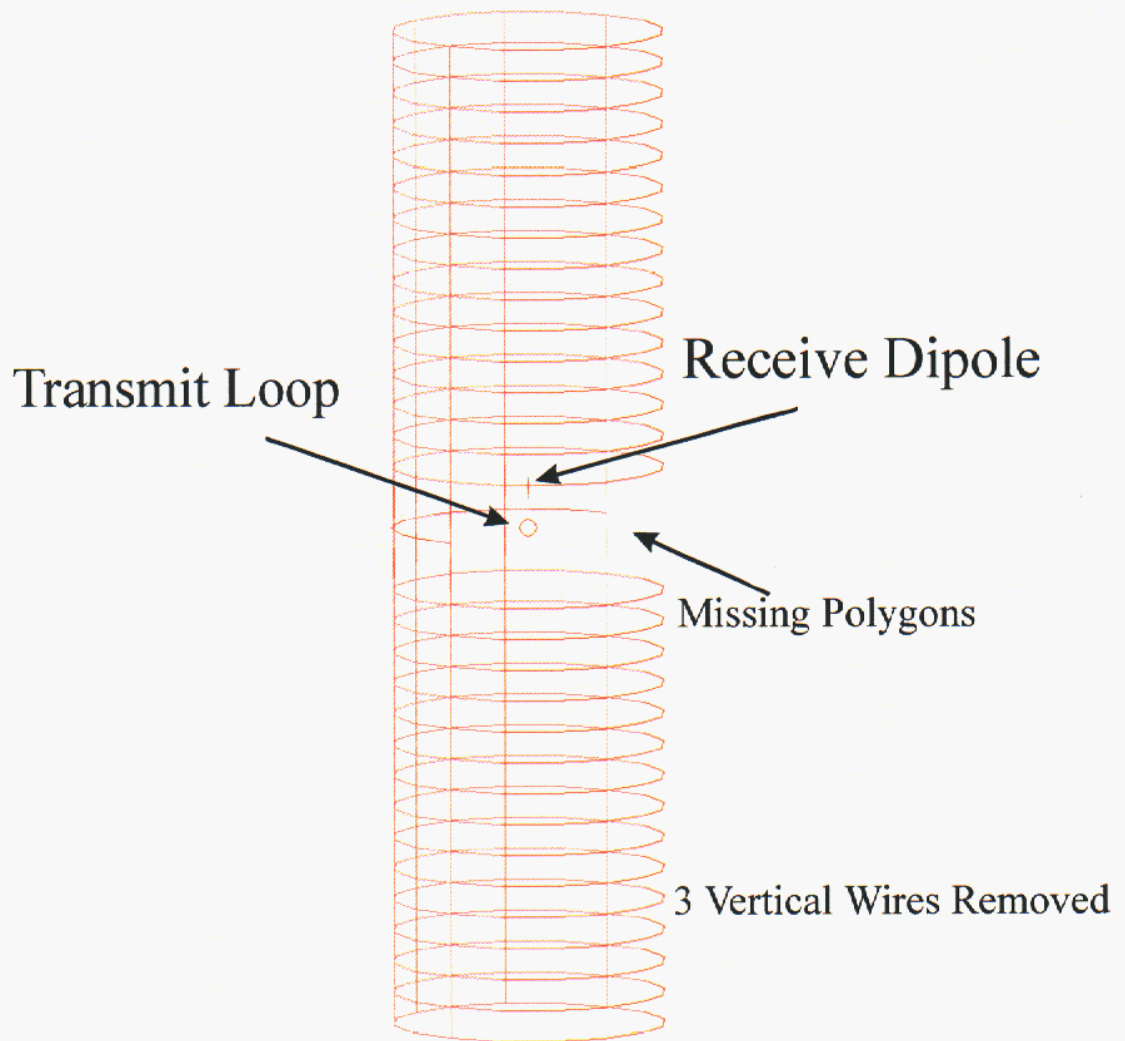

Figure 6-7. Wireframe Tube Model with Defects 
Segment Currents

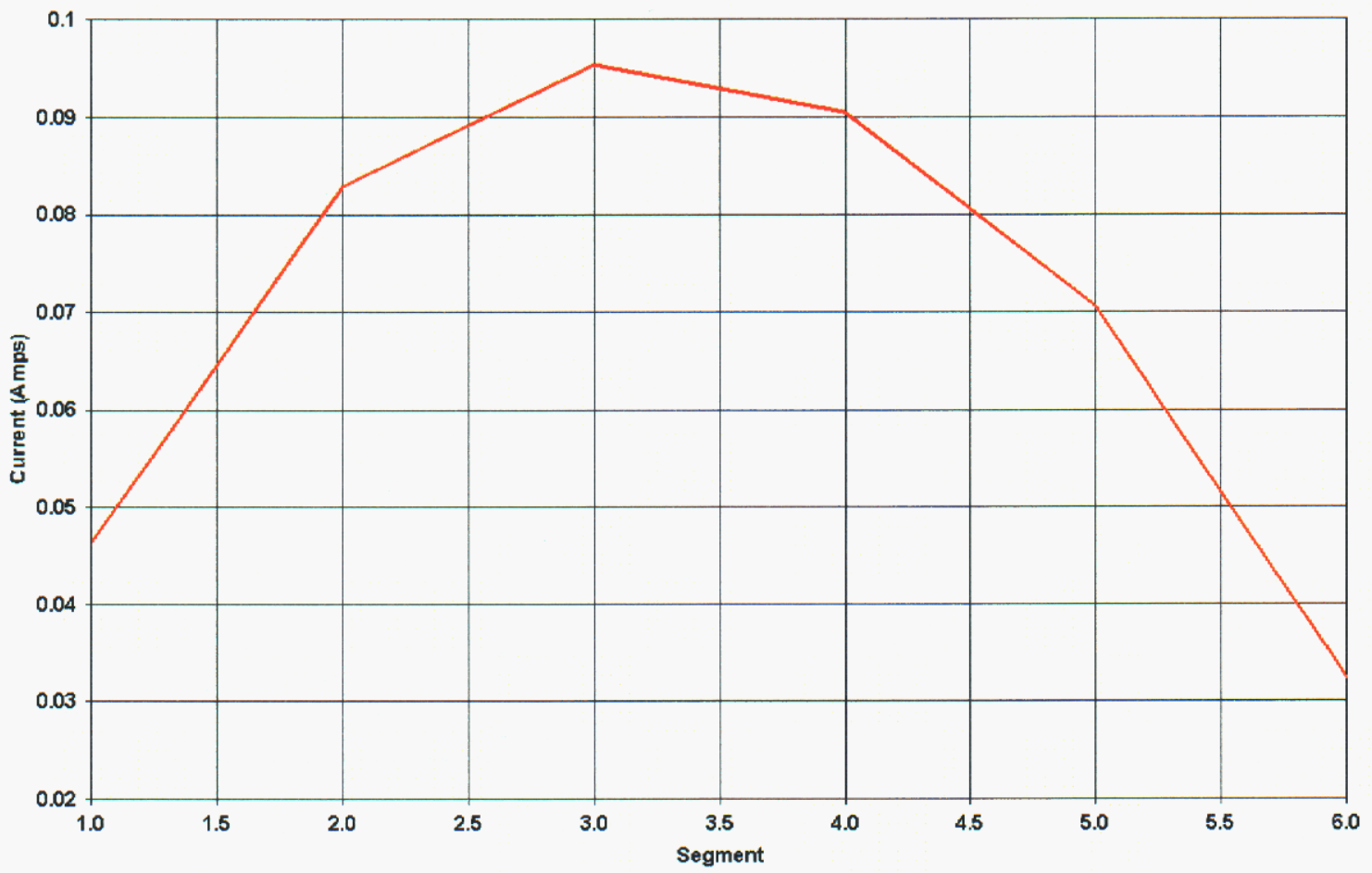

Figure 6-8. Current Distribution on Receive Dipole (No Defect)

Figure 6-9. Current Distribution on Receive Dipole (Defect) 
This page intentionally left blank 


\section{Conclusions and Recommendations for Future Work}

This three-year project centered on a very innovative concept that introduced a "radar" into a metallic tube to sense the integrity of the tube wall. It takes advantage of asymmetries that exist in the electromagnetic field distribution when defects are present. The initial vision of the project was to use computational modeling to numerically model and design the instrument. However, the computational modeling of the asymmetry in the signal was not as straightforward as was originally believed. Here we anticipated the merging of software developed at NMSU with government laboratory developed software and commercially available software. After some time it was concluded that the commercial software was not capable of analyzing the electromagnetic field at the scale that was needed for this project. The development of new software was beyond the scope of this project so at this point our efforts were refocused on developing the laboratory scaled-up prototype without the benefit of a detailed computational model.

A complete scaled up prototype of the device was designed and manufactured. In addition, an Inconnel test tube was purchased for the purpose of evaluating the probe. The following conclusions summarize our findings with respect to the laboratory testing of the concept:

- Our laboratory experimentation demonstrated that we can observe a signal change when an asymmetry is present in the tube wall (for example, part of the wall missing).

However, more work is needed to design a better probe that could detect small defects in the tube wall.

- The original concept was based on a transmitter and receiver design centered on the tube axis of symmetry. Our experiments indicate that much better results are obtained when the transmitter is adjacent to the tube wall. This change is significant in the theory of the operation of the device.

Our laboratory prototype development has resulted in development of a new controller for the probe driver and development of software for automated collection of the data. These developments will facilitate automation of the tube testing regardless of the probe technology; i.e., either in-tube radar or eddy current probe. Future enhancements to this software may include coupling to an automated defect detection software program which is being developed under DOE/NEPO/EPRI funding. This effort is promising because it could further enhance the current testing technologies.

The circuits and the prototype designed reflect contributions to fiber optics based communication with the probe. The use of fiber optics communication with the probe was necessary for the intube radar technology. Fiber optics communication may also enhance the current eddy-current devices by reducing the signal to noise ratio.

This work also resulted in significant investigation of the current state-of-the-art modeling in electromagnetics of the in-tube radar problem. There exist significant deficiencies in both commercial software as well as government software in modeling this electromagnetics problem. Perhaps a new code development effort focusing on this particular class of problem will result in a much better understanding of the physics of the in-tube radar problem and may provide some insight as to the difficulties that were encountered and why. The investigator team hopes to pursue this modeling effort in the future. 
This page intentionally left blank 


\section{References}

J. A. Stratton, 1941, “Electromagnetic Theory," McGraw-Hill, New York, pp 354-361, and p532.

James R. Wait and David A. Hill, 1977, "Electromagnetic Fields of a Dipole Source in a Circular Tunnel Containing a Surface Wave Line, " International Journal of Electronics, 1977, Vol. 42, No. 4, pp 377-391.

\section{REFERENCES}

1. Caffey, T. "Elements of a Continuous-Wave Borehole Radar," Technical Report SAND971995, Sandia National Laboratories, Albuquerque, NM, August 1997.

2. Caffey, T. "A Tool to Detect External Cracks from within a Metal Tube," Technical Report SAND97-0170, Sandia National Laboratories, Albuquerque, NM, August 1997.

3. Wait, J. R. and Hill, D. A., "Electromagnetic Fields of a Dipole Source in a Circular Tunnel Containing a Surface Wave Line", International Journal of Electronics, Vol. 42, No. 4, pp 377-391.

4. Jedlicka, R.P., Castillo, S.P., and Warne, L.K., "Coupling Through Tortuous Path Narrow Slot Apertures into Complex Cavities," IEEE Transactions on Antennas and Propagation, Volume 48, No. 3, pp. 456 - 466.

5. Lazarev, Y. N. et al. "Three-Dimensional Unified Codes for Solving the Forward Problem of Singlewell Electromagnetic Logging", Report on Task 4A of Contract AS-5553, Sandia National Laboratories, Albuquerque, NM, March 2000.

6. Brooks, S. "Continuous Wave In-Tube Radar: Development of an Experimental Prototype," M.S.M.E. thesis, New Mexico State University, Las Cruces, NM, 2000. 
This page intentionally left blank 


\section{Appendix A. Concept of the In-Tube Radar}

The basic idea is to position a transmitter and receiver within the hollow interior of a tube, and to transmit an electric field into the tube wall that will reflect from an anomaly and return to the receiver. It is assumed that that the interior of the tube is filled with air, and that the return from an anomaly, including a stress/corrosion crack on the outer surface, will be distinguishable from that of an acceptable part of the tube wall. A pulse-type radar system cannot be used because the small dimensions of boiler tubes, whose inner diameters are generally $10 \mathrm{~cm}$ or less, would require such a high frequency that the thickness of the metal wall would completely absorb the electromagnetic field before the field could reach the exterior.

A single-frequency, continuous-wave system, in which the transmitting and receiving antennas are designed to minimize the crosstalk between them, is presented here. The cross talk constitutes a 'self-clutter' that will set the lower bound to the signal-to-noise ratio of the system. The frequency must be low enough that the round-trip attenuation along the two-way path in the metal will be small enough to provide a detectable returned signal at the receiver. This attenuation constraint upon the frequency ensures that the cylindrical waveguide formed by the tube is operated below the cutoff frequency so that the receiver cannot be excited by a waveguide mode.

The sixteen possible combinations of elementary dipole antennas that could be used for a radar system within a small cylindrical tube were discussed in a Sandia report, and the appropriate antenna choices are as follows [Caffey, 1997]:

Transmitter -A magnetic dipole whose moment is oriented along the positive $\mathrm{X}$-axis, and centered upon the origin of coordinates where the $Z$-axis is coincident with the axis of the tube. This source provides an electric field component, $E_{Z}$, which is parallel to the Z-axis.

Receiver-An electric dipole, or disc-monopole probe, oriented along, and located upon, the Z-axis.

Assuming time-dependence as $\exp (j \omega t)$, the $E_{Z}$-field is given by:

$$
E_{Z}=\frac{-j \mu \omega I d A}{4 \pi} \sin \Theta \sin \Phi\left(\frac{1+j k R}{R^{2}}\right) e^{-j k R}, \quad \mathrm{~V} / \mathrm{m}
$$

where:

$$
\begin{aligned}
& j=\sqrt{-1} \\
& \mu=\text { permeability of free space, Henries } / \text { meter; } \\
& \omega=2 \pi f=\text { radian frequency, seconds }{ }^{-1} \\
& k=\alpha-j \beta=\text { the propagation factor, } \text { meters }^{-1}
\end{aligned}
$$$$
\text { with } \alpha=\frac{\omega \sqrt{\varepsilon_{r}}}{c} \cdot \sqrt{\frac{+1+\sqrt{1+g^{2}}}{2}} \text {, }
$$ 


$$
\begin{aligned}
& \beta=\frac{\omega \sqrt{\varepsilon_{r}}}{c} \cdot \sqrt{\frac{-1+\sqrt{1+g^{2}}}{2}}, \\
& g=\frac{\sigma}{\omega \varepsilon_{r} \varepsilon_{o}}=\text { the loss tangent; } \\
& \mathcal{E}_{o}=\text { the dielectric constant of free space, Farads } / \mathrm{m} ; \\
& \varepsilon_{r}=\text { the relative dielectric constant; } \\
& \sigma=\text { conductivity, Siemens } / \mathrm{m} ; \\
& c=\text { speed of electromagnetic propagation in free space, } \mathrm{m} / \mathrm{s} ; \\
& I=\text { dipole current, peak Amperes; } \\
& d A=\text { dipole area, meter } ;
\end{aligned}
$$

and $(R, \theta, \varphi)$ are the usual spherical coordinates. This formula for $E_{Z}$ is not defined at the origin, $R$ $=0$, where the magnetic dipole is located. The magnitude of $E_{Z}$ is a maximum in the plane $\Theta=$ $90^{\circ}$ and along the direction $\Phi= \pm 90^{\circ}$, and is zero whenever either $\Theta$ or $\Phi$ are either $0^{\circ}$ or $180^{\circ}$. In the Cartesian coordinates, $E_{Z}$ is a maximum along the $\pm Y$-axis and is zero in the $X Z$-plane aside from the origin.

When the magnitude of $E_{Z}$ is written-out, the terms in $R$ may be collected into one factor as shown below:

$$
\left|E_{z}\right|=\frac{\mu \omega I d A}{4 \pi}\left(\frac{\sqrt{(\alpha R)^{2}+(1+\beta R)^{2}}}{R^{2}} \exp (-\beta R)\right) \sin \theta|\sin \phi| \quad \mathrm{V} / \mathrm{m}
$$

If $\left|E_{Z}\right|$ is normalized by its maximum value, the relative distribution of $\left|E_{Z}\right|$ depends only on the $(\sin \Theta \cdot|\sin \Phi|)$ - product. That is, the angular distribution of $\left|E_{Z}\right|$ is the same for any spherical radius greater than zero, and is independent of both the operating frequency and the media parameters.

This distribution is shown in Figure 1 in which the half-power beam width of each lobe is $90^{\circ}$, and the two lobes are out-of-phase with each other. The bi-lobed distribution describes the primary field even when a cylindrical metal tube encloses the magnetic dipole.

The basic concept is that a very small electric antenna, sensitive to only the Ez-field, and placed anywhere in the $X Z$-plane, aside from the origin, would be completely uncoupled from the transmitter. However, the electric antenna will always have a finite cylindrical dimension, and its effect on self-clutter will be examined later. 


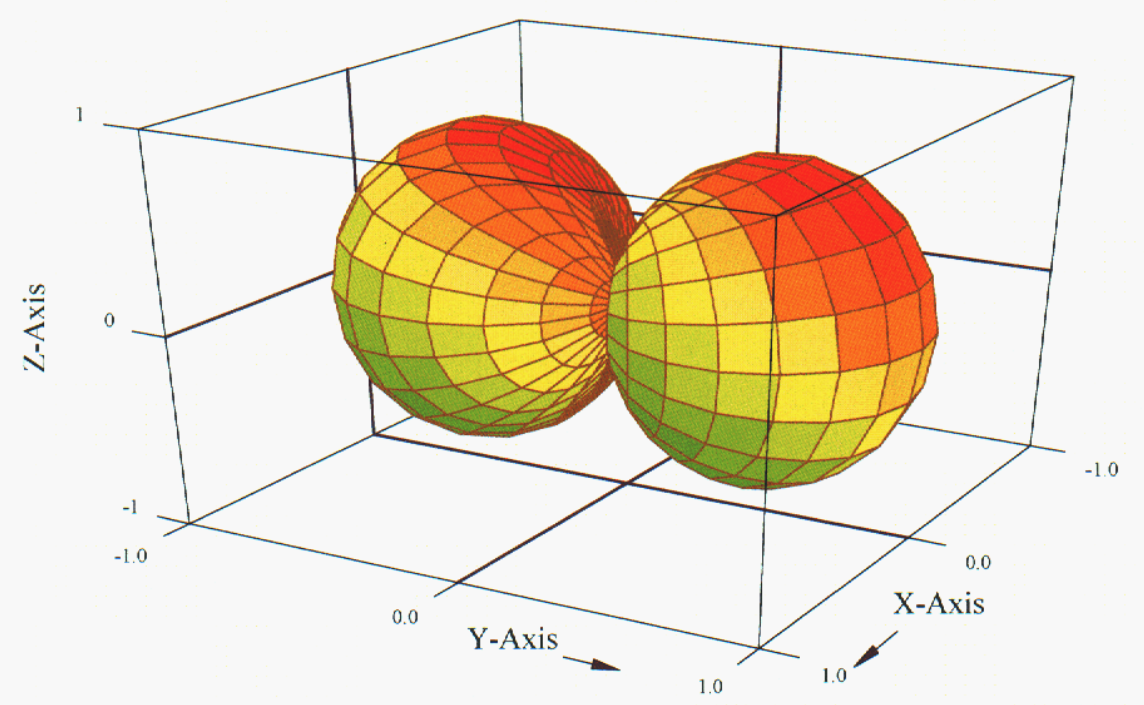

Figure 0-1. Relative distribution of the $E_{z}-$ field from an $X$-directed magnetic dipole

Crosstalk will occur when either of the two antennas is displaced from the ideal location because the geometry will be unsymmetrical. The distortion of the fields when the two antennas are translated together parallel to the Z-axis will be shown in the next section.

David Hill and the late Jim Wait developed the solution for the electromagnetic fields within an infinitely long cylindrical void surrounded by a complex homogeneous medium, and applied it to communication in a circular tunnel [Wait and Hill, 1977]. The theory is summarized below with the authors' notation in which time-harmonic fields are assumed to vary as $\exp (j \omega t)$.

Figure 2 depicts the XY-plane intercepted by a circular cylinder of radius ' $a_{o}$ ' whose axis is coincident with the $\mathrm{Z}$-axis. A magnetic dipole, with its moment parallel to the $\mathrm{X}$-axis, is located at cylindrical coordinates $\left(\rho_{l}, \varphi_{l}, 0\right)$ where $\rho_{l}<a_{0}$, and the field point is at $(\rho, \varphi, z)$ with $\rho \leq a_{0}$. The cylinder is air-filled with permeability $\mu_{O}$ and dielectric constant $\varepsilon_{O}$, and the surrounding homogeneous media has conductivity $\sigma_{e}$ with permeability $\mu_{e}$ and dielectric constant $\varepsilon_{e}$.

The $E_{Z}$ field is represented as a superposition of modes in terms of the Hertz potentials that supply the modal contributions:

$$
E_{Z}=\sum_{m=-\infty}^{+\infty} \int_{-\infty}^{+\infty}-v^{2}\left\{A_{m}(\lambda) K_{m}(v \rho)+P_{m}(\lambda) I_{m}(v \rho)\right\} \exp \left[-j m\left(\phi-\phi_{1}\right)\right] \exp (-j \lambda z) d \lambda
$$

where $I_{m}(v \rho)$ and $K_{m}(v \rho)$ are modified Bessel functions, $v^{2}=\lambda^{2}+\gamma_{o}^{2}$ and $\gamma_{o}=j \omega / c$. The functions $A_{m}(\lambda)$ and $B_{m}(\lambda)$ are the primary electric and magnetic Hertz potentials determined by the strength and orientation of the magnetic dipole source, and $P_{m}(\lambda)$ is the secondary potential that is related to $A_{m}(\lambda)$ and $B_{m}(\lambda)$ by the boundary conditions at the cylinder/media interface. 


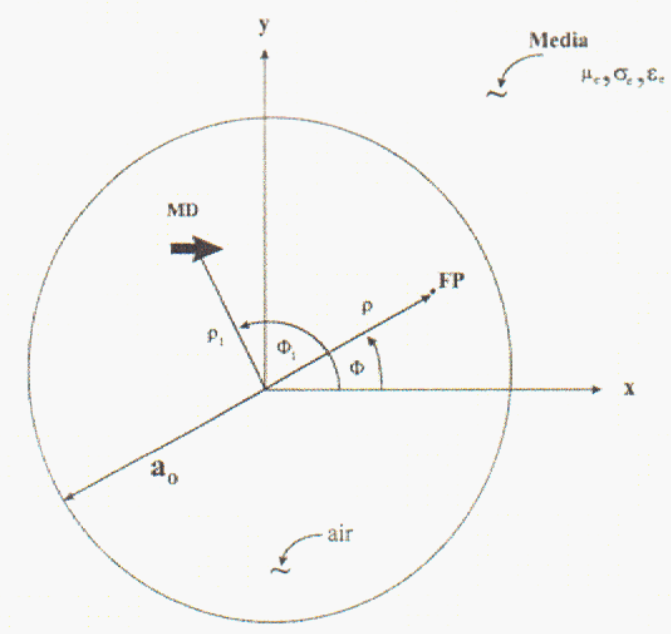

Figure $\mathbf{0 - 2}$. Cross-section geometry for a cylindrical void

When the X-directed magnetic dipole is located at $Z=0$, the primary Hertz potentials are given by these expressions when $\rho_{l}<\rho$ :

$$
A_{m}(\lambda)=\frac{-\mu_{o} \omega I d A}{8 v \pi^{2}}\left[I_{m-1}\left(v \rho_{1}\right) \exp \left(-j \phi_{1}\right)-I_{m+1}\left(v \rho_{1}\right) \exp \left(j \phi_{1}\right)\right] \quad \mathrm{V} \bullet \mathrm{m}^{2}
$$

and

$$
B_{m}(\lambda)=\frac{-j \lambda I d A}{8 v \pi^{2}}\left[I_{m-1}\left(v \rho_{1}\right) \exp \left(-j \phi_{1}\right)+I_{m+1}\left(v \rho_{1}\right) \exp \left(j \phi_{1}\right)\right] \quad \mathrm{A} \bullet \mathrm{m}^{2}
$$

The boundary conditions at the cylinder/media interface can be succinctly expressed in terms of a radial wave impedance $Z_{\mathrm{m}}$ and a radial wave admittance $\mathrm{Y}_{\mathrm{m}}$ at $\rho=a_{o}$ [Stratton, 1941]:

$$
\begin{aligned}
& E_{\phi m}=\alpha_{m} E_{z m}+Z_{m} H_{z m} \\
& H_{\phi m}=-Y_{m} E_{z m}+\alpha_{m} H_{z m}
\end{aligned}
$$

where:

$$
\begin{aligned}
& \alpha_{m}=m \lambda /\left(a_{o} u^{2}\right) \\
& \gamma_{e}^{2}=j \mu_{e} \omega\left(\sigma_{e}+j \omega \varepsilon_{e}\right) \\
& u^{2}=\lambda^{2}+\gamma_{e}^{2} \\
& Y_{m}=\left[j \gamma_{e}^{2} /\left(u \mu_{e} \omega\right)\right] K_{m}^{\prime}\left(u a_{o}\right) / K_{m}\left(u a_{o}\right),
\end{aligned}
$$

and

$$
Z_{m}=-\left(j \mu_{e} \omega / u\right) K_{m}^{\prime}\left(u a_{o}\right) / K_{m}\left(u a_{o}\right)
$$


The application of the boundary conditions establish the relation of the secondary potential, $P_{m}$, to the primary potentials $A_{m}$ and $B_{m}$ :

$$
P_{m}=-\left[A_{m}(\lambda) r_{m}+B_{m}(\lambda) t_{m}\right] / D_{m}, \quad \mathrm{~V} \bullet \mathrm{m}^{2}
$$

where:

$$
\begin{gathered}
r_{m}=\left[\left(\frac{m \lambda}{a_{o} v^{2}}-\alpha_{m}\right)^{2}+\left(\frac{\gamma_{o} K_{m}^{\prime}}{v K_{m}}+\eta_{o} Y_{m}\right)\left(\frac{\gamma_{o} I_{m}^{\prime}}{v I_{m}}+\frac{Z_{m}}{\eta_{o}}\right)\right] I_{m} K_{m}, \\
t_{m}=\left(\frac{m \lambda}{a_{o} v^{2}}-\alpha_{m}\right) \frac{j \mu_{o} \omega}{a_{o} v^{2}}, \\
D_{m}=\left[\left(\frac{m \lambda}{a_{o} v^{2}}-\alpha_{m}\right)^{2}+\left(\frac{\gamma_{o} I_{m}^{\prime}}{v I_{m}}+\eta_{o} Y_{m}\right)\left(\frac{\gamma_{o} I_{m}^{\prime}}{v I_{m}}+\frac{Z_{m}}{\eta_{o}}\right)\right] I_{m}^{2},
\end{gathered}
$$

and $\eta_{0}=\sqrt{\mu_{0} / \varepsilon_{0}}$ is assumed to be the characteristic impedance of the air inside the cylinder. The argument of the modified Bessel functions in Eq (13-16) is $a_{o} v$, and the prime, ', denotes differentiation with respect to $v$, the wave number within the cylinder. This theory is applied to the computation of source-to-receiver crosstalk in the next section. 
This page intentionally left blank 


\section{Appendix B. Crosstalk Computations}

Before the possibility of detecting a stress-corrosion crack on the outside of the tube can be considered, it is necessary to examine the crosstalk between the source and receiver within the tube itself. The crosstalk must be reduced to a small level in order to detect anomalies when the ITR is misaligned with the tube centerline.

The parameters of the experimental work performed by NMSU are repeated here in terms of the computational quantities listed in the previous section, namely:

$f=10 \mathrm{kHz}$, the operating frequency;

$a_{o}=4.445 \mathrm{~cm}$, or 1.75 inches, the inner radius of the Inconel pipe; and

$\sigma_{e}=8.2 \mathrm{E} 5 \mathrm{~S} / \mathrm{m}$, the conductivity of the Inconel pipe.

Both the relative permeability and the relative dielectric constant of the tube are taken as unity, The dimensions of the disc-monopole used as the $\mathrm{E}_{Z}$ - field probe are needed to compute the open-circuit potential available to the receiver input, and are listed below.

Diameter: 0.020 inches $(0.508 \mathrm{~mm})$;

Length: $\quad 0.25$-to- 0.5 inches $(6.35$-to- $12.7 \mathrm{~mm})$; and

Separation distance from source centerline to probe tip: 10,20 , or $30 \mathrm{~mm}$.

Only the most favorable geometry is considered here, namely that the probe is at the maximum separation distance (to reduce crosstalk) and has the maximum length (to increase signal detection). The probe is always parallel to the $Z$-axis and extends from $30 \mathrm{~mm}$ to $42.7 \mathrm{~mm}$. The distribution of the magnitude of the total $E_{Z}$-field in the XY-plane near the midpoint of the probe for this symmetrical case is shown in Figure B-1. The crosstalk is zero because both source and receiver are coaxial with the centerline of the tube. Figure B-2 is a similar plot for an unsymmetrical location of the source and receiver, namely $\rho_{l}=\rho=0.05 \bullet$ Tube Radius, and $\phi_{l}=$ $\phi=90^{\circ}$.

Computations were made only for the case where the two antennas were aligned parallel to the $Z$ axis and translated together along the $+Y$-axis. Only this parallel offset case was investigated because the general case of tilting the line joining the antennas would require the addition of a $Y$ directed source dipole. This would be a straightforward modification, but does not appear necessary at this time. Because the dimensions of the probe are extremely small in terms of the air-wavelength within the tube, the surface must be an equipotential. The potential was computed by multiplying the mean value of Ez-field on the surface on the probe by the length of the probe. The length of the probe was divided into five azimuth planes, spaced $2.54 \mathrm{~mm}$ apart, and in each plane the azimuth angles were varied in eight increments of 45 degrees upon the surface of the probe. 


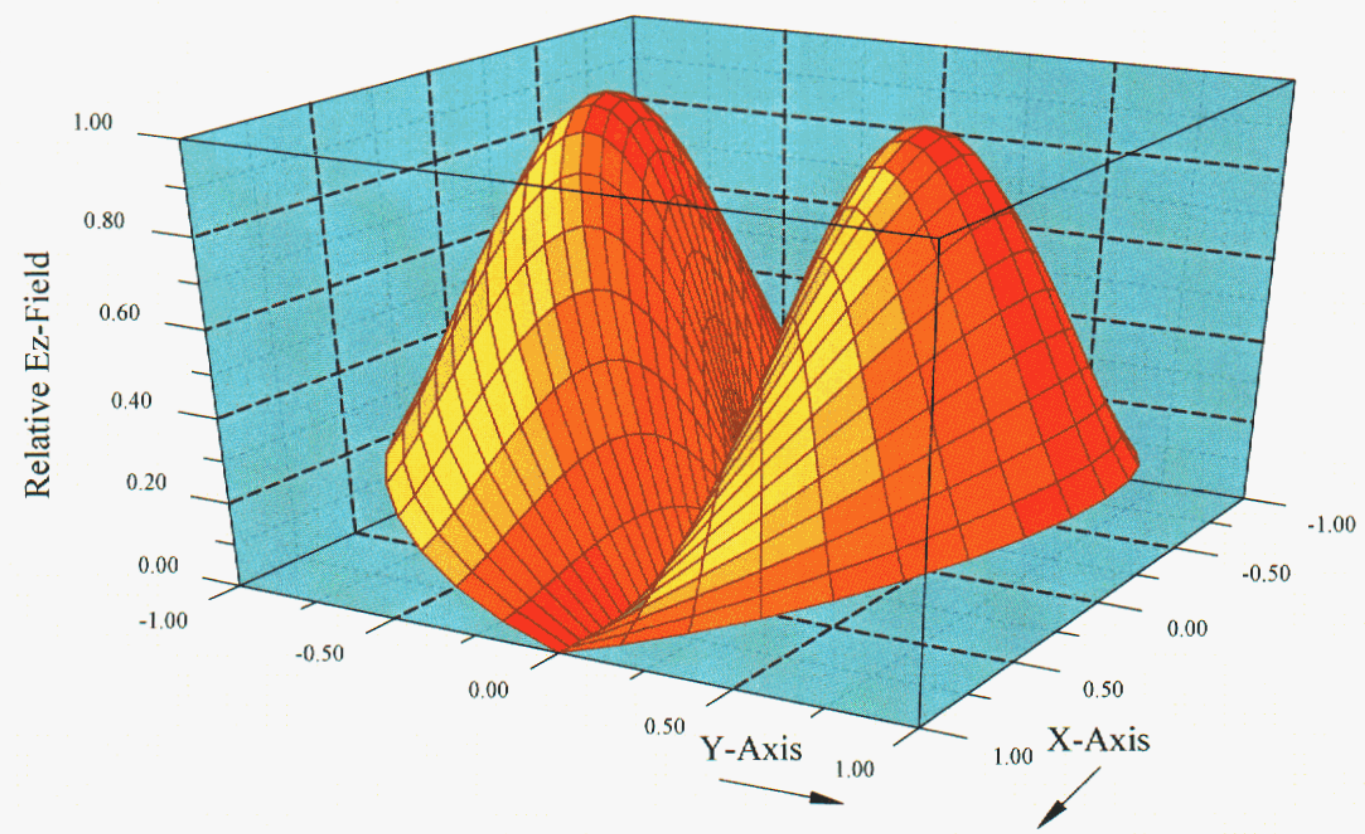

Figure 0-1. Distribution of the total $\mid E_{Z}$-field $\mid$ near the probe midpoint. The source is located at the origin of coordinates

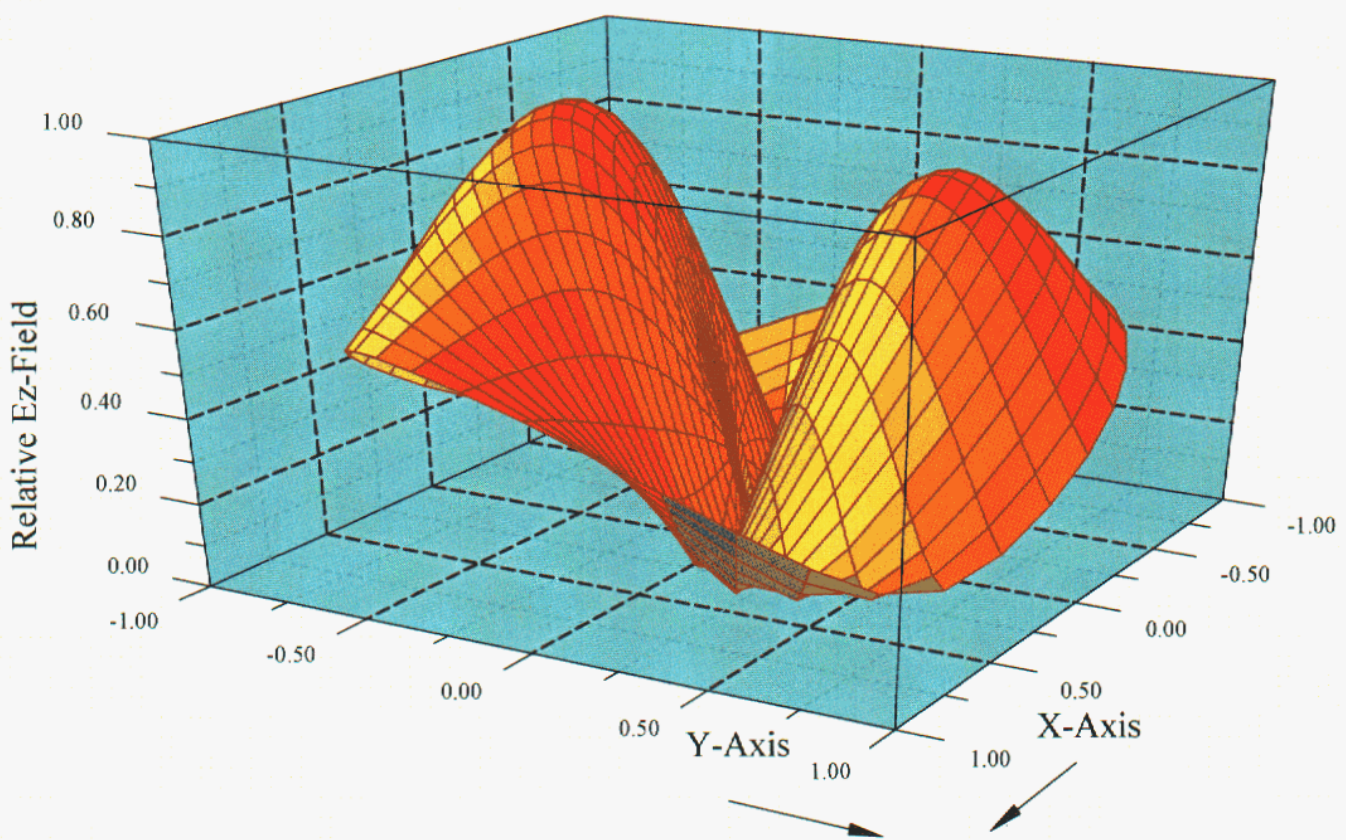

Figure 0-2. Distribution of the total $\mid E_{Z}$-field| near the probe midpoint The source is located at $5 \%$ of the tube radius on the $+Y$-axis

The distortion of the $E_{Z}$ - field along the for the two source locations, centered and offset, is seen more clearly in Figure B-3 than from comparison of the surface plots. 


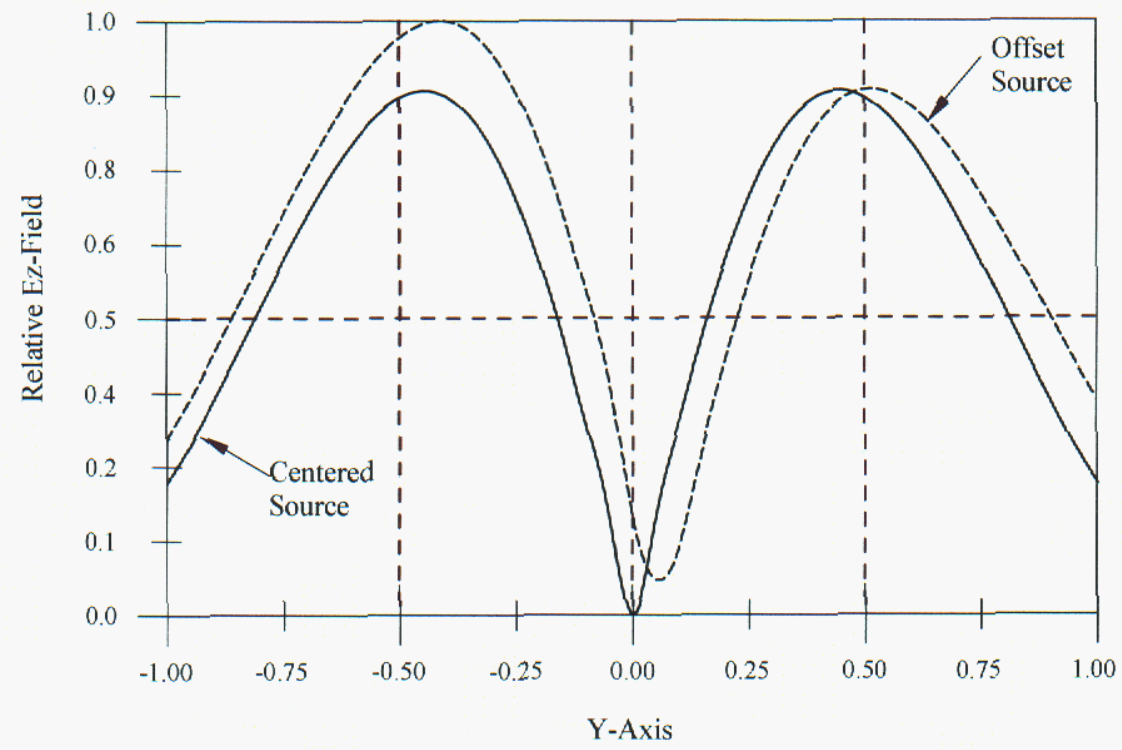

Figure 0-3. The effect of source location upon. The distribution the $E_{z}$-field along the $Y$-axis

The radial offset for the first computation was chosen to place the surface of the probe tangent to the $Z$-axis. The following table illustrates that the crosstalk increases with the radial offset as expected.

\begin{tabular}{c|c|c|c}
\multicolumn{4}{c}{ Crosstalk Summary } \\
Run \# & Radial Offset,mm & $\begin{array}{c}\text { (Radial Offset) } \\
\text { (Tube Radius) }\end{array}$ & $\begin{array}{c}\text { Induced Potential } \\
\text { peak } \mu \text { Volts }\end{array}$ \\
\hline 1 & 0.2540 & $0.571 \%$ & $3.2 @ 128 \mathrm{deg}$ \\
2 & 0.4445 & $1 \%$ & $5.2 @ 229 \mathrm{deg}$ \\
3 & 0.8890 & $2 \%$ & $19 @ 110 \mathrm{deg}$ \\
4 & 1.3335 & $3 \%$ & $45 @ 102 \mathrm{deg}$ \\
5 & 1.7778 & $4 \%$ & $72 @ 100 \mathrm{deg}$ \\
6 & 2.2225 & $5 \%$ & $100 @ 99 \mathrm{deg}$
\end{tabular}

Because a unit dipole moment of one-Ampere $\bullet$ meter $^{2}$ was assumed in the computations, the crosstalk potentials must be scaled to the actual moment that is designed for the ITR. Conversely, the dipole moment must be chosen so that, at some misalignment specification, the crosstalk is much less than the thermal noise of the receiver electronics. 


\section{DISTRIBUTION}

1 Bahram Nassersharif

Department of Mechanical Engineering

New Mexico State University

PO Box 30001

Las Cruces, NM 88003-8001

1 Gabe V. Garcia

Department of Mechanical Engineering

New Mexico State University

PO Box 30001

Las Cruces, NM 88003-8001

1 Russell P. Jedlicka

Klipsch School of Electrical and Computer Engineering

New Mexico State University

PO Box 30001

Las Cruces, NM 88003-8001

20 MS0748 G.E. Rochau, 6863

1 MS0750 T.W.H. Caffey, 6116

1 MS9018 Central Technical Files, 8945-1

2 MS0899 Technical Library, 9616

2 MS0612 Review \& Approval Desk, 9612 for DOE/OSTI 